\title{
Photonic Crystals Modified by \\ Optically Resonant Systems
}

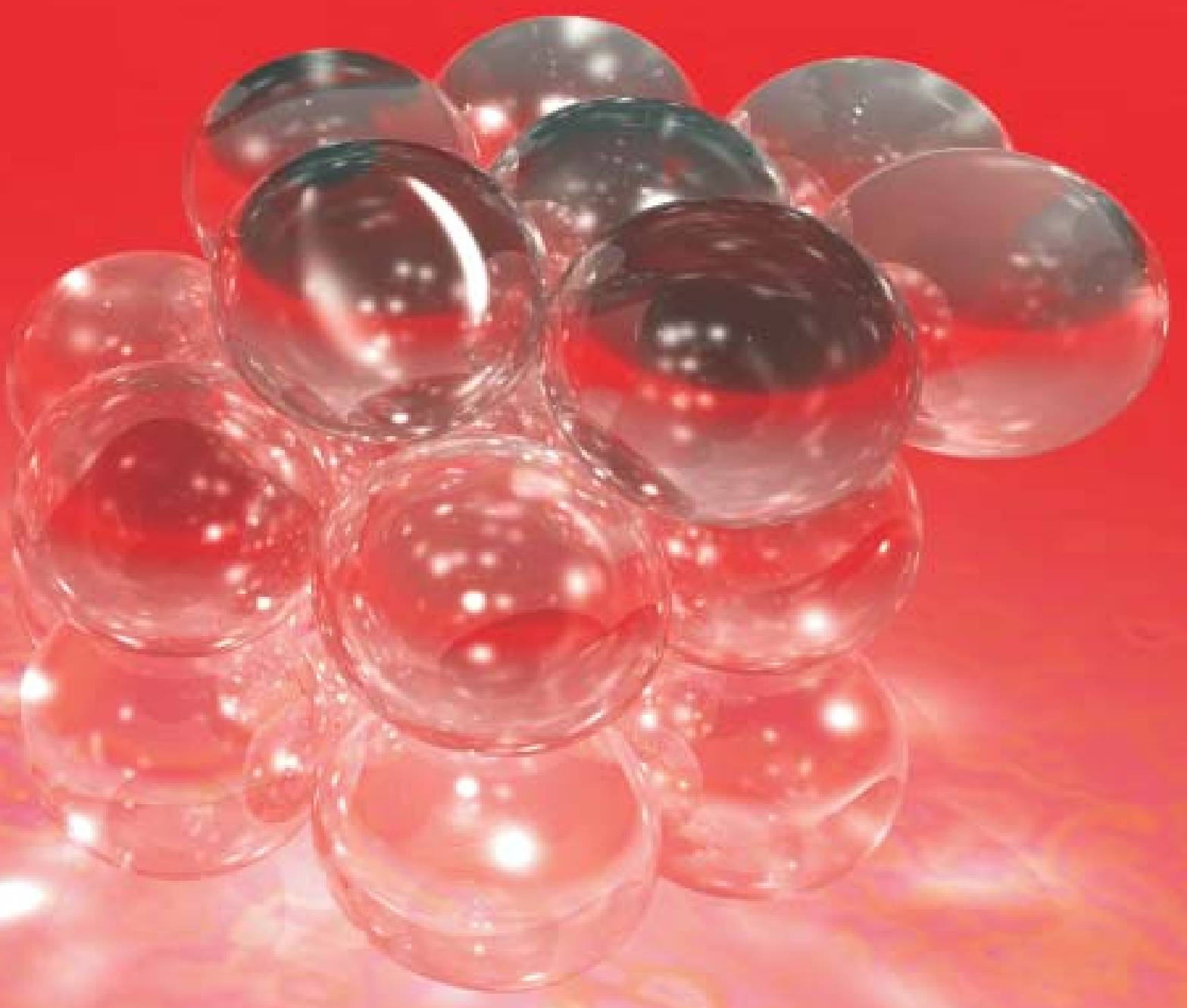

Philip J. Harding 


\section{PHOTONIC CRYSTALS MODIFIED BY OPTICALLY RESONANT SYSTEMS}


Promotiecommissie

$\begin{array}{ll}\text { Promotor } & \text { prof. dr. W. L. Vos } \\ \text { Assistent Promotor } & \text { dr. A. P. Mosk } \\ \text { Overige leden } & \text { prof. dr. H. J. Bakker } \\ & \text { prof. dr. K. J. Boller } \\ & \text { prof. dr. H. Kurz } \\ & \text { dr. P. W. H. Pinkse } \\ & \text { prof. dr. M. Pollnau }\end{array}$

Paranimfen

T. Warnaar

E. A. Harding

The work described in this thesis is part of the research program of the 'Stichting voor Fundamenteel Onderzoek der Materie (FOM)', which is financially supported by the 'Nederlandse Organisatie voor Wetenschappelijk Onderzoek (NWO)'.

This work was carried out at the Complex Photonic Systems Group, Department of Science and Technology and $\mathrm{MESA}^{+}$Institute for Nanotechnology,

University of Twente, P.O. Box 217, 7500 AE Enschede, The Netherlands. and at the FOM Institute for Atomic and Molecular Physics (AMOLF), Kruislaan 407, 1098SJ Amsterdam, The Netherlands, where a limited number of copies of this thesis is available.

Cover: Artist's impression of an fcc opal immersed in an atomic vapor (rendered by PovRay).

This thesis can be downloaded from www.photonicbandgaps.com. Printed by Print Partners Ipskamp, Enschede, The Netherlands (2008). ISBN: 978-90-365-2683-8 


\section{PHOTONIC CRYSTALS MODIFIED BY OPTICALLY RESONANT SYSTEMS}

\section{PROEFSCHRIFT}

ter verkrijging van

de graad van doctor aan de Universiteit Twente,

op gezag van de rector magnificus,

prof. dr. W.H.M. Zijm,

volgens besluit van het College voor Promoties

in het openbaar te verdedigen

op donderdag 13 juni 2008 om 15.00 uur

door

Philip James Harding

geboren op 28 januari 1977

te Hamburg 
Dit proefschrift is goedgekeurd door:

prof. dr. W. L. Vos en dr. A. P. Mosk 
Past tears are present strength.

- George MacDonald, Phantastes 

To Adi. 



\section{Contents}

1. Introduction 13

1.1. Photonic crystals . . . . . . . . . . . . . . . 13

1.1.1. Nanophotonics . . . . . . . . . . . . . . 13

1.1.2. What is a photonic crystal ? . . . . . . . 13

1.1.3. Shall I compare thee to a semiconductor ? . . . . . . . 14

1.1.4. Bragg diffraction in photonic crystals . . . . . . 15

1.1.5. Fabrication of photonic crystals . . . . . . . . . 18

1.1.6. External probes of real photonic crystals . . . . . . . 19

1.2. Optical resonances in photonic crystals . . . . . . . . . 21

1.2.1. Free carriers . . . . . . . . . . . . . . 22

1.2.2. Atomic resonances . . . . . . . . . . . . . . . 24

1.2.3. Cavities . . . . . . . . . . . . . . 25

1.3. This thesis . . . . . . . . . . . . . 26

2. Experimental setup and alignment 29

2.1. Pump and probe beams . . . . . . . . . . . . . 29

2.2. Broadband detection . . . . . . . . . . . . . . . . 32

2.3. Frequency-resolved detection . . . . . . . . . . 34 
3. Dynamical ultrafast all-optical switching of planar GaAs/AIAs photonic microcavities

3.1. Introduction . . . . . . . . . . . . . . . 35

3.2. Experimental setup: sample and linear reflectivity . . . . 35

3.3. Results: dynamic cavity resonance . . . . . . . . . . 36

3.3.1. Linear reflectivity . . . . . . . . . . . . . . 36

3.3.2. Dynamic reflectivity . . . . . . . . . . . . . . 39

3.3.3. Dynamic carrier density and refractive index . . . . . 42

3.4. Results: Dynamic linewidth . . . . . . . . . . . . . 44

3.4.1. Linear reflectivity . . . . . . . . . . . . . . 44

3.4.2. Dynamic linewidth . . . . . . . . . . . . . 45

3.5. Conclusions and recommendations . . . . . . . . . . 49

4. Dynamics of light in an ultrafast switched cavity 53

4.1. Introduction . . . . . . . . . . . . . . 53

4.2. Experimental setup and linear reflectivity . . . . . . . 54

4.3. Data processing . . . . . . . . . . . . . 54

4.4. Negative probe delay . . . . . . . . . . . . . . . 55

4.4.1. The intuitive model . . . . . . . . . . . . 58

4.5. Positive probe delay . . . . . . . . . . . . . . 58

4.6. Physical mass and string model . . . . . . . . . . . . 59

4.7. Results: mass and spring . . . . . . . . . . . . 61

4.7.1. Static case . . . . . . . . . . . . . . 62

4.7.2. Dynamic case . . . . . . . . . . . . . . 62

4.8. Delimitations . . . . . . . . . . . . . . . 63

4.9. Conclusions and recommendations . . . . . . . . . 64

5. Femtosecond versus picosecond switching of Si woodpile photonic $\begin{array}{ll}\text { crystals } & 67\end{array}$

5.1. Introduction . . . . . . . . . . . . . . . 67

5.2. Experimental setup and sample . . . . . . . . . . . 68

5.3. Linear reflectivity . . . . . . . . . . . . . . . . . 69

5.4. Switched reflectivity vs. delay at one probe frequency . . . 70

5.5. Switched reflectivity vs. frequency . . . . . . . . . 71

5.5.1. Reflectivity vs. frequency at coincidence . . . . . . 71

5.5.2. Reflectivity vs. frequency at $\Delta t=1 \mathrm{ps} \ldots \ldots \ldots .72$

5.5.3. Model: the extended scalar wave approximation . . . 73

5.5.4. Interpretation of spectra at coincidence . . . . . . 75

5.5.5. Interpretation of ps switching . . . . . . . 78 
5.6. Reflectivity vs. pump frequency at coincidence . . . . . . 79

5.7. Non-degenerate instantaneous figure of merit . . . . . . . 80

5.8. Conclusions and recommendations . . . . . . . . . . 82

6. Enhancement and reduction of an opal's photonic strength by $\begin{array}{ll}\text { atomic dispersion } & 85\end{array}$

6.1. Introduction . . . . . . . . . . . . . . 85

6.1.1. Atoms in photonic crystals . . . . . . . . . . 85

6.1.2. ${ }^{133} \mathrm{Cs}$ - the ideal resonator . . . . . . . . . . 87

6.1.3. Cs as a strongly photonic medium . . . . . . . . 89

6.1.4. $\mathrm{SiO}_{2}$ opals as photonic media . . . . . . . . . . 94

6.2. Experimental setup . . . . . . . . . . . . . . . . 94

6.2.1. Excitation of ${ }^{133} \mathrm{Cs} \ldots \ldots \ldots$. . . . . . . . 94

6.2.2. Control of the vapor . . . . . . . . . . . . . 96

6.2.3. Samples . . . . . . . . . . . . . . . . . . 97

6.3. Results and Discussion . . . . . . . . . . . . . . . 99

6.3.1. Reflection at Cs resonances . . . . . . . . . . . . 99

6.3.2. Reflection of the opal . . . . . . . . . . . . 100

6.4. Conclusions and recommendations . . . . . . . . . . . . 104

A. Derivation of the position dependent effective dielectric constant of a colloid monolayer

$\begin{array}{ll}\text { Nederlandse samenvatting } & 109\end{array}$

$\begin{array}{ll}\text { Dankwoord } & 115\end{array}$

$\begin{array}{ll}\text { Bibliography } & 119\end{array}$ 



\section{Introduction}

\subsection{Photonic crystals}

\subsubsection{Nanophotonics}

The early $19^{\text {th }}$ century witnessed an industrial revolution regarding the use of mechanical energy for use in various labor intensive industries, most notably textile fabrication. Similarly, semiconductors contributed much to the way of life in the late $20^{\text {th }}$ century, by making large-scale computing power and ubiquitous mobile communication accessible. The $21^{\text {st }}$ century has been hailed as an era in which light is harnessed to solve some of mankind's most pressing problems. Nanophotonics is a branch of physics that has interfaces with solar cell research, optical computing, telecommunications and many more disciplines. At the very heart of nanophotonics lies the ability to control light with intricate nanostructures, such as metal-hole arrays, negative-index materials, nanowires, and random and ordered photonic materials [1-5]. Prominent feats that can be achieved are converting light into material waves and back again to light [6], strongly confining light and slowing it down [7], and molding the flow of light [8], which can be achieved with photonic crystals.

\subsubsection{What is a photonic crystal ?}

For a structure to be called a photonic crystal, is has to meet three requirements. First, the crystal must have a spatially varying dielectric constant that varies periodically with a period of the order of a wavelength of light. Second, the amplitude of this spatially varying dielectric constant is required to be large, of the order unity. Third, the absorption in the structure has to 
be limited in order to allow for multiple light scattering. Therefore, metals and certain semiconductors are unsuitable ingredients in photonic crystals, for despite their high real dielectric constant, the absorption is appreciable at optical frequencies. In atomic crystals such as quartz, the dielectric constant changes on length scales comparable to short wavelengths in the X-ray range. However, at these frequencies the amplitude is less than $10^{-4}$, and therefore atomic crystals are hardly photonic. In order to achieve sufficient dielectric contrast then, photonic crystals are always composite structures. Finally, the first requirement discards all disordered systems such as milk, foam, and clouds.

\subsubsection{Shall I compare thee to a semiconductor?}

When these conditions are fulfilled, photonic crystals are said to act as semiconductors for light [9]. When the wavelength of the incoming light matches a lattice spacing of the crystal, the waves are reflected by interference, and collectively give rise to a Bragg peak, also known from X-ray diffraction [10]. The width of the Bragg peak is proportional to the difference in dielectric constant $\Delta \epsilon$. This wide Bragg peak is dubbed stopband, as its prevents a finite bandwidth of light to propagate in the direction normal to the lattice planes. Surprisingly, the width of a stopband was determined already in 1887 by Lord Rayleigh, albeit for one dimensional laminar structures [11]. In analogy, the width of the electronic bandgap in a semiconductor is equal to the potential difference seen by the electrons, and so the potential seen by light can be related to $\Delta \epsilon$. For a judicious choice of crystallographic structure, and a sufficiently high dielectric contrast, a photonic bandgap can be obtained, a region of frequencies for which light propagation is completely inhibited $[12 ; 13]$. Photonic bandgap materials are widely pursued in the community $[14 ; 15]$. For these structures, the density of optical states (DOS) vanishes and spontaneous emission is forbidden [16]. While partial modification of spontaneous emission has been demonstrated for 2 and 3D photonic crystals [17-20], complete inhibition by a 3D bandgap crystal is a much awaited feat in the scientific community.

However, photonic crystals differ from semiconductors in three important respects: Because photons are bosons, they are not subject to the Pauli exclusion principle. Any number of photons can occupy each available mode. Therefore, photons have no Fermi energy. Second, the total number of electrons is conserved in any low-energy process $(<0.5 \mathrm{MeV})$, while this is not the case for photons. Therefore, absorption and non-linear processes become 

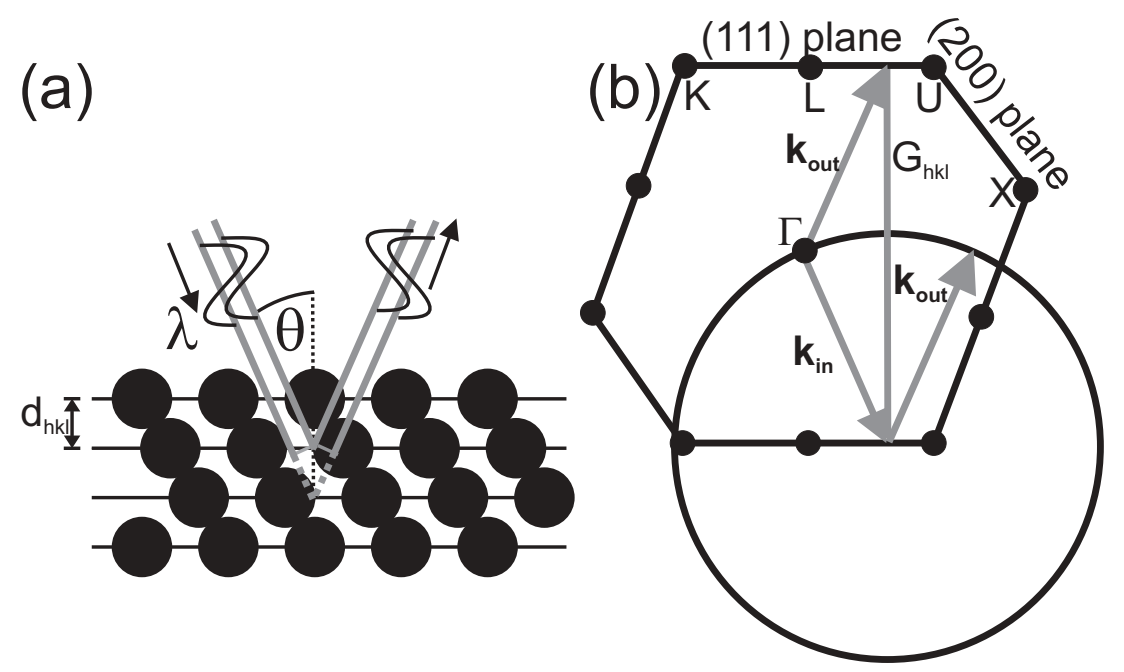

Figure 1.1.: (a) Schematic representation of Bragg diffraction from the [111] lattice planes of an fcc crystal in real space. When the wave incident with angle $\theta$ and of wavelength $\lambda$ in vacuum scatters from a family of planes with spacing $d_{h k l}$, the scattered waves interfere constructively when the path difference (dashed lines) equals a multiple of the wavelength. (b) Reciprocal space representation of Bragg diffraction. When the incoming wave with wavevector $\mathbf{k}_{\text {in }}$ and the scattered wave with wavevector $\mathbf{k}_{\mathrm{out}}$ obey $\mathbf{k}_{\mathrm{out}}-\mathbf{k}_{\mathrm{in}}=\mathbf{G}$, where $\mathbf{G}$ is reciprocal lattice vector, then Bragg diffraction occurs. The Ewald sphere and a cross-section of the Brillouin zone of an fcc crystal have also been drawn.

important when dealing with photons. Third, while the depth of the electron potential is given by a combination of the Coulomb field of the atoms and the electrons' kinetic energy [21], the photons' potential is proportional to the square of their frequency $[22 ; 23]$. Therefore, a photon with a low frequency will be subject to a negligible potential, whereas a low-energy electron is always trapped. In other words, the long wavelength associated with the low frequency is much longer than any period of the structure. Caution must therefore be exercised in comparing photonic crystals to semiconductors.

\subsubsection{Bragg diffraction in photonic crystals}

Bragg diffraction is central to this Thesis, and we shall elaborate two common representations of Bragg diffraction, both of which shall be used. Figure 1.1(a) represents Bragg scattering from the [111] planes of a face-centered cubic (fcc) photonic crystal in real space [21]. Electromagnetic waves with wavelength $\lambda$ are incident under an angle $\theta$ on an fcc photonic crystal. The waves scatter from successive planes with spacing $d_{h k l}$. When any multi- 
ple of the wavelength equals the path difference between two lattice planes, the waves will interfere constructively. We have drawn the reciprocal space representation of the same situation in figure 1.1(b). A wave with wavevector $\mathbf{k}_{\text {in }}$ is incident from the origin of the Brillouin zone, and scatters to a wavevector $\mathbf{k}_{\text {out }}$. To compare to the previous picture, $\left|\mathbf{k}_{\text {in }}\right|=\left|\mathbf{k}_{\text {out }}\right|=2 \pi / \lambda$. The von Laue condition requires that $\mathbf{k}_{\text {out }}-\mathbf{k}_{\text {in }}=\mathbf{G}_{h k l}$ for constructive interference, where $\mathbf{G}_{h k l}$ is a reciprocal lattice vector, $\left|2 \pi / \mathbf{G}_{h k l}\right|=d_{h k l}$. We see that $\mathbf{k}_{\text {out }}-\mathbf{k}_{\text {in }}$ is indeed a reciprocal lattice vector, namely $\mathbf{G}_{111}$. Bragg diffraction thus occurs for $\left|\mathbf{k}_{\text {in }}\right|=\left|\mathbf{k}_{\text {out }}\right|=\pi / d_{h k l}$.

For photonic crystals, Bragg's law has to be corrected for the change in wavelength the waves undergo in the photonic crystal [24]. Then, Bragg's law reads

$$
\lambda=2 d_{h k l} n_{\mathrm{eff}} \cos (\theta),
$$

where $n_{\text {eff }}=\sqrt{\bar{\epsilon}}=\sqrt{\phi \epsilon_{1}+(1-\phi) \epsilon_{2}}$ is an effective refractive index. Here, $\phi$ is the volume fraction of the material of dielectric constant $\epsilon_{1}$, and $\epsilon_{2}$ is the dielectric constant of the second material.

The difference between $\epsilon_{1}$ and $\epsilon_{2}$ gives rise to stopgaps, frequency ranges which forbid propagation of light in a given direction. This forbidden propagation can best be represented in a band diagram, which shows the dispersion relation between the wavevector $\mathbf{k}$ and the frequency $\omega .{ }^{1}$ In figure $1.2(\mathrm{a})$, we plot such a dispersion relation in the direction $\Gamma-L$, shown in figure 1.1(b). At low frequencies, the dispersion relation is $\omega=k c / n_{\text {eff }}$, where $c$ is the celerity of light in free space. Close to the Bragg condition $k=\pi / d$ however, the dispersion relation opens up, and no frequencies exist in the frequency interval $\Delta \omega$ around $\omega_{0}$, where $\omega_{0}$ is the frequency corresponding to $\lambda$ (see equation 1.1), $\omega_{0}=\pi c /\left(d n_{\text {eff }}\right)$ at normal incidence. The relative magnitude of the gap width can be estimated from following argumentation [8]: At normal incidence, $\lambda=2 d_{h k l} n_{\text {eff }}$. At this wavelength, the incoming and scattered wave interfere constructively, and two standing waves form. One of these waves has antinodes in the low index material, while the field extrema of the other wave are located primarily in the high index material. There are now two standing waves with wavevector $\mathbf{k}_{\text {in }}=-\mathbf{k}_{\text {out }}$ at two different refractive indices. Therefore, these standing waves will have different frequencies. The width of the gap will be related to the difference $\epsilon_{2}-\epsilon_{1}$, and the relative width to $\left(\epsilon_{2}-\epsilon_{1}\right) / \sqrt{\bar{\epsilon}}$. Using diffraction theory [25], one can

\footnotetext{
${ }^{1}$ We will denote peaks in reflectivity spectra by stopbands, while the gaps in associated with certain directions are of the bandstructure are stopgaps.
} 
derive $[26]$

$$
S=\frac{\Delta \omega}{\omega_{0}} \approx \frac{\left|\epsilon_{1}-\epsilon_{2}\right|}{\bar{\epsilon}}\left|f_{\mathbf{G}_{h k l}}\right|,
$$

where $f_{\mathbf{G}_{h k l}}$ is the first Fourier component associated with the spatial distribution of $\Delta \epsilon$. The width of the stopband provides an experimentally accessible gauge for the crystal's photonic strength $S$ [27]. We conclude that photonic crystals strongly modify the propagation of light, and especially so for frequencies around the stopgap.

Because of the modes' difference in relative antinode position, the phase velocity, or structural refractive index $c k / \omega$ will be markedly different at both edges of the gap. We have plotted the structural refractive index $n$ of a photonic crystal in figure 1.2(b). For better visibility, $n_{\text {eff }}$ has been subtracted from the real part $n^{\prime}$. Outside the gap, the change in structural index is 0 . At the red edge of the gap however, the index increases, in agreement with the onset of the dielectric standing wave. Throughout the gap, $k=G / 2$, but the frequency increases. At the blue edge of the gap, $n^{\prime}-n_{\text {eff }}$ is negative, but recovers to 0 at large positive detunings. Experimental evidence of modified structural index has been demonstrated by analysis of the resonances of Fabry-Pérot fringes close to a stopband [28; 29]. The imaginary part $n^{\prime \prime}$ describes the removal of energy from the incident beam. Already in 1914, Darwin noted that the energy removal by an atomic crystal was around 100 higher than that expected from 'true absorption of the crystal' [30]. At the gap center, $n^{\prime \prime}$ is maximum, and it vanishes at the gap edges. A beam transmitted through a photonic crystal will thus be most attenuated at the gap center, while outside the gap, there is no attenuation. The behavior of both $n^{\prime}$ and $n^{\prime \prime}$ is strongly reminiscent of resonances. In analogy with the strength of an atomic resonance, the strength and therefore the width of this resonance has been associated with the polarizability per unit volume $[27 ; 31]$.

In the case of a photonic crystal, the polarizability is caused by structural properties. High material polarizabilities can be achieved by resonant single atoms. Indeed, there has been considerable interest to form a photonic crystal from highly polarizable atoms, see e.g. [32]. Here it was shown that a bandgap opens for atoms with a sufficiently high resonator strength combined with a high filling fraction in the lattice. Resonant atoms in photonic crystals will be discussed in detail in Chapter 6 . 


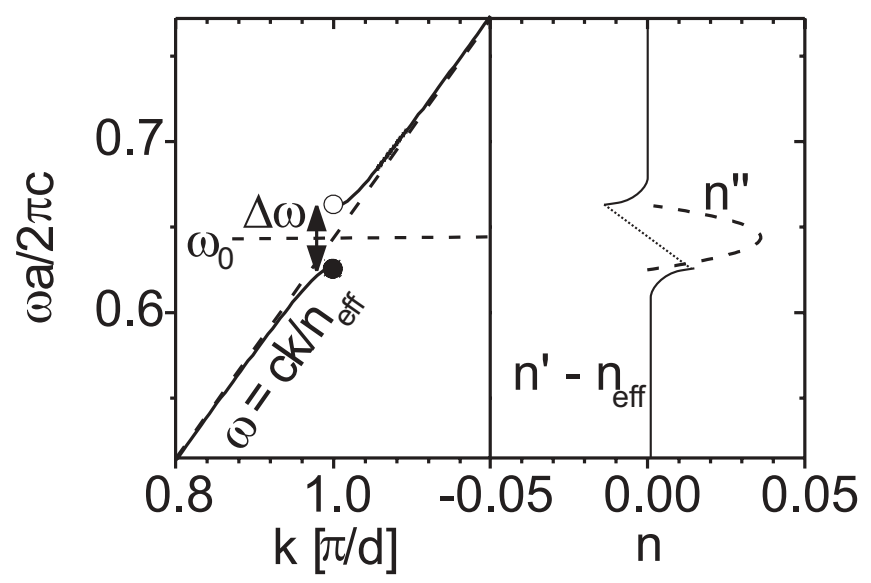

Figure 1.2.: (a) Dispersion relation for a $\mathrm{SiO}_{2}$ opal, calculated in the $\Gamma-L$ direction. The air mode and dielectric mode are given by $\circ$ and $\bullet$, respectively. (b) Structural real and imaginary refractive index of a photonic crystal.

\subsubsection{Fabrication of photonic crystals}

Even in the initial proposal of photonic crystals, Yablonovitch suggested that 'further material development' would be necessary before 'the benefits [of photonic crystals] are fully felt' [16]. Indeed, is has proven challenging to fabricate periodic structures with $\mu \mathrm{m}$ sized periods, while maintaining a sufficiently index contrast. After Yablonovitch's proposal to use fcc crystals, research suggested that fcc crystals would have no bandgap [12;33; 34]. Sözuer et at. later demonstrated that fcc crystals could have a bandgap in the range of $2^{\text {nd }}$ order diffraction [13]. This crystal would consist of air spheres in a high dielectric background with a refractive index contrast in excess of $m=2.8$.

Ho et al. proposed that a diamond symmetry is eligible for a photonic bandgap [12]. The bandgap was predicted to occur at lower refractive index contrast of only $m=2.0$ for fcc. The first bandgap crystal was fabricated by mechanically drilling holes into a high-dielectric (n' $=3.6)$ to form a crystal with a diamond symmetry [35]. The bandgap was in the microwave region owing to the large dimensions of the holes. Other crystals possessing diamond symmetry are the woodpile crystals [36; 37], where pairs of dielectric rods are stacked upon one another orthogonally, the $(n+2)$ th layer being offset by half a period to the nth layer. Recently, several authors succeeded in downscaling the rods from $\mathrm{cm}$ to sub $\mu \mathrm{m}$, yielding bandgaplike behavior in the near infrared at telecom wavelengths $[15 ; 38]$. A SEM image of such a 
woodpile photonic crystal can be seen in figure 1.3(a). Inverse woodpiles are shown figure 1.3(d): here, cylinders were etched and milled into a crystalline silicon substrate [39].

A popular method to fabricate fcc photonic crystals is to have polystyrene or silica colloids suspended in a liquid self-assemble to form opals by letting the suspension evaporate [40-42]. A scanning electron microscopy (SEM) image is shown in figure 1.3(b). Originally, large 2D arrays had been fabricated in a similar manner by [43]. Thick crystals can be made by sedimentation $[24 ; 44]$, although disorder combined with the large length scales in these thick crystals limits the optical quality, evidenced by the disappearance of Fabry-Pérot fringes in reflection or transmission. These self-assembled opals have an fcc structure, evidenced by small angle X-ray diffraction [45], and their (111) surface is oriented towards the substrate surface. Opals are amenable to inversion with precursors of high-dielectric semiconductors [46], and thus bandgap crystals can be formed [47;48].

Molecular Beam Epitaxy (MBE) has proven a popular method for fabricating Bragg stacks, as well as 1- and 2D microcavities, i.e., cavities embedded in photonic crystals $[49 ; 50]$. The Bragg stacks consist of III-IV semiconductors, often GaAs and AlAs. Here, pieces of ultrapure gallium and arsenic are heated until they start sublimating. In ultrahigh vacuum, the atoms diffuse to a GaAs substrate where they condense and react to form crystalline GaAs, owing to the low flow rate. For the AlAs layers, aluminium is used. The layer thickness can be controlled to less than a monolayer, and so the GaAs and AlAs layers can be made of variable thickness, which is opportune for the growth of microcavities. These planar microcavities can be etched to micropillars, structures of high-Q factors $(Q=150000)$ owing to the additional lateral confinement [51]. Figure 1.3(c) shows such a micropillar.

\subsubsection{External probes of real photonic crystals}

In this section, we will briefly discuss ways to measure optical properties of real photonic crystals from the outside. Internal probes form a fascinating subject in itself $[17 ; 52 ; 53]$, but will not be studied here. In contrast, reflectivity is a well suited method for probing the bandstructure of photonic crystals externally [54]. A focussed broad- or narrowband beam is incident on the sample under a given angle, and the irradiance of spectral distribution of the reflected beam is measured. Reflectivity can give access to the bandstructure by associating peaks in the measured spectrum $[29 ; 54-57]$, although care has to be exercised in identifying reflectivity peaks with gaps: 

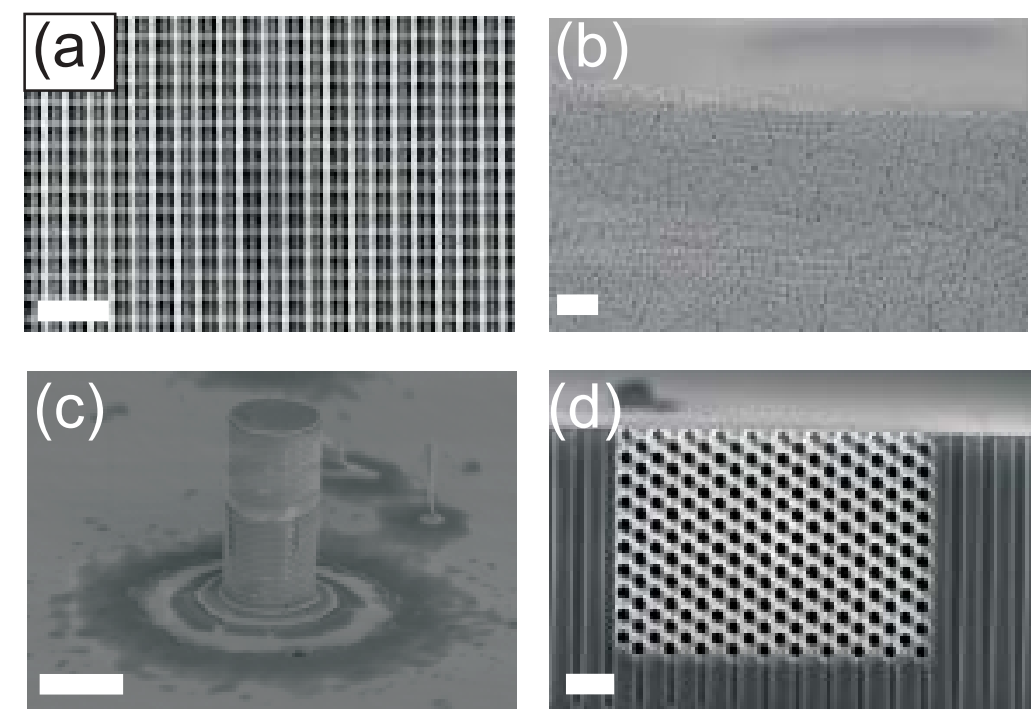

Figure 1.3.: Scanning electron microscopy images of different photonic crystals. (a) Top view of a woodpile photonic crystal [38] used in the measurements presented in Chapter 5. (b) Top view of a thin $\mathrm{SiO}_{2}$ opal, Chapter 6. (c) GaAs/AlAs micropillar, $\lambda / 4$ Bragg stacks sandwiching a cavity. (d) Inverse woodpile, cylinders milled and etched into crystalline Si. Images courtesy of Léon Woldering (a), Yoanna-Reine Nowicki-Bringuier (c), and R. Willem Tjerkstra (d). The scale bar represents $2 \mu \mathrm{m}$.

while every gap in the bandstructure gives rise to a reflectivity peak, not every peak is due to a gap.

In the absence of disorder, the height of the reflectivity peak due to a stopgap depends on both photonic strength and crystal thickness $L$. The length scale associated with the photonic strength is the Bragg length $L_{B}$, the length over which the transmitted beam has decayed to $1 / e$ of its original value [26],

$$
L_{B}=\frac{\lambda}{\pi S}
$$

Ideally, the reflectivity is then given by

$$
R=1-\exp \left(-L / L_{B}\right)
$$

Since this thesis shall be concerned with induced changes in optical properties of photonic crystals, there is a need to justify why reflectivity is so well suited. We give two reasons:

1. In real photonic crystals, the ideal Bragg length is modified by disorder [58-60]. While the transmissivity depends on all lattice planes, 
reflectivity depends mostly on the first $L_{B} / d_{h k l}$ planes. Because the disorder length $\ell_{\text {ext }}$ is by definition much longer than $L_{B}$, but not necessarily smaller than $L$, the reflectivity peaks will be less subject to disorder, evidenced in peak broadening and general smearing out of spectral features. Hence transmission studies intending on measuring the stopband width are only useful if $\ell_{\text {ext }}>L$. The modulation depth and thus the gradient $d T / d \omega$ of transmissivity at the edge of peaks is thus reduced with respect to those of reflectivity peaks. The reduced modulation depth also diminishes the change in transmissivity due to induced changes in the photonic crystal. Therefore, reflectivity is also better suited than transmissivity to probe the change in optical properties. If one does want to measure changes in transmission due to the changes in optical properties of one of the composites, Sigalas et al. recommended studying thin crystals if these are extincting [61].

2. Many real photonic crystals are fabricated on substrates. Reflectivity of thick photonic crystals with $L>L_{B}$ will be insensitive to scattering and absorption of the substrate, in contrast to transmissivity.

From these two arguments we conclude that reflectivity measurements sensitively probe changes in photonic crystals' optical properties. However, transmissivity studies have proven useful in studying photonic crystals' diffusive properties [56], and served as a basis for the first bandstructure measurements $[62 ; 63]$. Other external reflecting probing methods, such as near field microscopy, are not appropriate for our purposes [64].

\subsection{Optical resonances in photonic crystals}

Resonances ares widely pursued in optics, because they allow the propagation of light to be modified [65], and even for it to be trapped [66]. As a result, the dispersion relation for light is modified compared to free space propagation. An example of resonant and dispersive behavior is apparent in the photonic gap in fig. 1.2(a). In this section, we shall discuss what effect various kinds of resonances have on the optical properties of the photonic crystal. We consider three kinds of resonances: a., free carrier resonances, b. bound electron resonances, i.e., atomic resonances, and c. cavity resonances. For all these cases, we will show how the Bragg resonance is modified, and will present the change in dispersion. 


\subsubsection{Free carriers}

\section{General properties of free carriers in semiconductors}

The well-known Drude model relates the motion of free electrons to the induced refractive index [21]. One of its great successes lies in the excellent experimental agreement of metals' electrical conductivity with frequency. In the Drude model, the motion of an electron due an applied electric field is randomized after an elastic collision. The time between these collisions is referred to as the Drude damping time $\tau_{D}$. The resonance condition is governed the density dependent restoring force of the ions in the lattic [67]. The Drude model can be extended to account for holes, and can then describe optical properties of photoexcited free carriers in semiconductors [68]. The total refractive index of the excited semiconductor is a sum of the contribution due to bound electrons and that of the free carriers. However, it should be realized that the the initial transient of a dense electron-hole plasma's behavior is complex and is actively being studied [69-71].

The timescales of the generation and the recombination is short: the generation is largely limited by the pump pulse duration, while the recombination time depends on surface and bulk properties, and varies from ps to $\mu \mathrm{s}$. Because of the fast timescales, the term switching is appropriate.

Another important property of carriers in semiconductors is that both the carrier generation cross-section and their dielectric response are strongly frequency dependent $[72 ; 73]$. Therefore, degenerate switching, in which the probe beam is the same as the pump beam [74; 75], will have profoundly different effects from non-degenerate switching. To allow for greater flexibility, we have chosen an experimental setup which uses two independently tunable lasers. Therefore, degenerate switching that is notably used for vertical-cavity light-emitting diodes and saturable absorbers will not be discussed $[76 ; 77]$.

\section{What is switched?}

We can distinguish three different implementations of switching. Each instance employs generation of free carriers in semiconductors, but the desired outcome is entirely different. The first use of ultrafast free carrier generation was to switch the propagation of light, by changing the transmission or reflection of an incident beam. Already in 1979, Gibbs et al. succeeded in switching a GaAs Fabry-Pérot cavity, and suggested using it as an optical modulator [78]. The recombination times were downscaled by 9 orders of 
magnitude 10 years later [79]. The advent of high-Q resonators has lowered the power requirement of the pump pulse to achieve any given change in transmittance (see, e.g., [80]). Recently, propagation switching of high-Q circular resonators was achieved [81;82], and dynamic pulse delaying was shown [66]. In Chapter 3 we will provide experimental evidence for propagation switching via cavity resonances in microcavities. The second, most heralded implementation is the change in DOS, which might ultimately lead to ultrafast modification of spontaneous emission rates [83]. Here, four requirements are discussed: (i), a large change in $n^{\prime}$ with (ii) concomitant low $n^{\prime \prime}$, (iii) the short timescale in which the switch should take place, and (iv) the spatial homogeneity of the carrier distribution. Surprisingly, the lack of homogeneity leads to non-adiabatic dynamics of light, predicted by [84] and demonstrated by [85]. In Chapter 5, we will experimentally derive a nondegenerate homogeneity relation which has importance for DOS switching. The third instance is to investigate how electromagnetic fields respond to quickly changing dielectric surroundings. Recent demonstrations of bandwidth changes $[86 ; 87]$ and adiabatic and non-adiabatic response of pulses in cavities [88] have been achieved. In Chapter 4, we experimentally study the electromagnetic field that is subject to a fast changing cavity resonance.

\section{Dispersion of free carriers and the effect on the photonic gap}

Free carriers give rise to a dispersion as shown in figure 1.4(b). At low frequencies, the magnitude of both $n^{\prime}$ and $n^{\prime \prime}$ is large, but they decay with the square and cube of the detuning, respectively. The restoring force exerted by the ions on the free carriers causes a resonant frequency of the plasma, the plasma frequency. Above this frequency, the index is largely real. In figure 1.4(a), the dispersion relation for the unswitched photonic crystal is shown (see figure 1.2(a)). The switched refractive index causes both the Bragg diffraction condition and the photonic strength to be modified, and causes both $\omega_{0}$ and $\Delta \omega$ to change. A decrease in the high index material $n_{\text {high }}$ blue shifts the Bragg condition (eq. 1.1), and simultaneously narrows the gap. The largest change in reflectivity will be evident at the red edge of the gap. This relation can be generalized: for a change in $n_{\text {high }}$, the largest change in reflectivity will be observed at frequencies corresponding to the dielectric mode, whereas the largest change in reflectivity will be observed at the air mode for a change in $n_{\text {low }}$. Switching is wont to exclusively generating carriers in the low bandgap material, as selective excitation in the high gap material is impossible by optical means. From Moss' rule, which empirically 


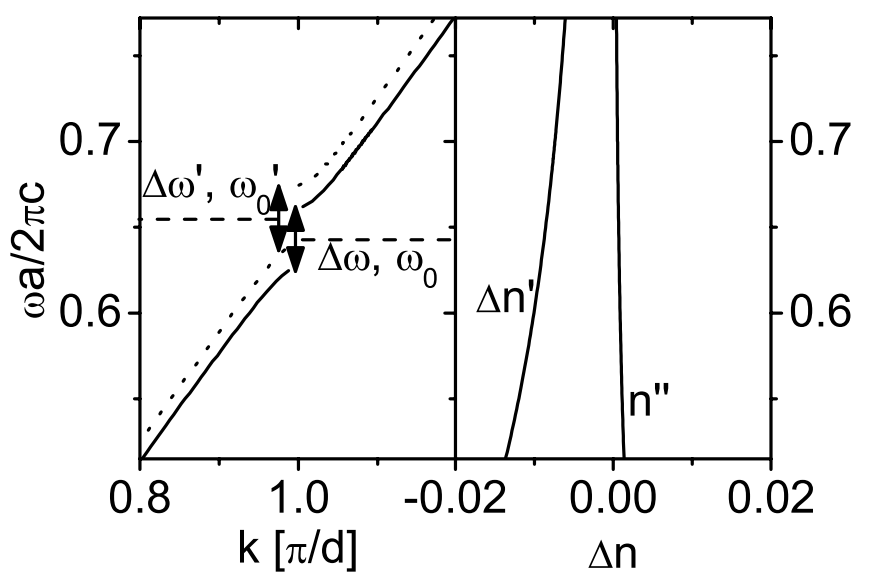

Figure 1.4.: (a) Dispersion relation for an unswitched photonic crystal (solid curve), and a photonic crystal with free carriers in the backbone (dashed curve). Both the stopgap frequency and the stopgap width change due to the presence of free carriers. (b) Change in real $\left(\Delta n^{\prime}\right)$ and imaginary part $\left(\Delta n^{\prime \prime}\right)$ of the material refractive index of free carriers relative to the structural refractive index of the unswitched crystal. The carrier density was set to $N=1 \cdot 10^{25} \mathrm{~m}^{-3}$, corresponding to a plasma frequency of 0.16 in $\omega a /(2 \pi c)$.

relates the bandgap to $n^{\prime-4}$ [89], we therefore conclude that we always modify the high index material, and large reflectivity changes will always be observed at the red edge.

\subsubsection{Atomic resonances}

A different implementation of a resonance in a photonic crystal is a narrow resonance of atoms. Atomic resonances can give rise to drastically altered propagation conditions [65]. The atoms have bound outer electrons, which behave very much like a mass on a spring: while the elongation is in phase with the driving field below resonance, the elongation leads the driving field above resonance. It is the interplay between this phase change and extinction of the incident wave that gives rise to refraction. This seemingly simple model proposed by $\mathrm{H}$. A. Lorentz at the eve of $20^{\text {th }}$ century captures most of the essential physics of many everyday optical phenomena [90]. Consider for example a prism: Upon irradiation with white light, the light separates into its different color components, an experiment famously performed by Newton. This separation takes place because each color component has a 
different velocity, and will thus propagate under a different angle according to Snell's law. Rainbows work in very much the same way. The dispersion also dictates the sub unity dielectric constant of all materials at X-ray frequencies.

In contrast to Drude electrons, the bound electrons are usually subject to a much larger restoring force, and thus have a higher resonance, and in our case in the near infrared. The second difference to the Drude electron is that the width of the resonance is given by radiative damping, although at higher vapor densities collision broadening becomes dominant. In our case the width is only $6 \cdot 10^{-5}$ that of the stopgap width. The third distinction is that the change in the refractive index takes place in the low index material. Therefore, the changing $n^{\prime}$ will change the reflectivity most when the atomic resonance is at the blue edge of a stopgap. The fourth dissimilarity is that the Drude dispersion induces a negative refractive index change with respect to a high $n$ background, whereas the atomic resonance can cause both $n^{\prime}>1$ or $n^{\prime}<1$.

Figure 1.5(b) shows the real and imaginary parts of an atomic resonance. The resonance width has been grossly enlarged for enhanced visibility. At the red edge of the resonance, $n^{\prime}>1$. Using the simple dispersion relation $\omega=k c / n_{\text {eff }}$, an increasing $n^{\prime}$ must be met by an increased $k$ for any given

$\omega$. The change in reflectivity caused by such a dispersive and absorptive medium in a photonic crystal is complex, and shall be dealt with in Chapter 6 .

\subsubsection{Cavities}

While the previous two sections discussed alteration of the Bragg resonance by material dispersion, this section shows how defects engineered into otherwise periodic photonic crystals modify the structural dispersion [16]. A cavity is a defect with a size comparable to the wavelength of visible light. Such cavity - photonic crystal systems (microcavities) have attracted considerable interest because of their high $\mathrm{Q}$ factors combined with low mode volumes, giving rise to high Purcell factors. Indeed, these microcavities have been shown to modify spontaneous emission [20; 91]. High field enhancements in the cavity have been predicted to amplify non-linear effects [92]. It is perhaps not surprising that cavity-atom systems have been subject to much interest [93].

Under given conditions, this microcavity structure supports a mode in the stopgap, see figure 1.6(a). This mode is characterized by a quality factor $Q$, and its resonance $\omega_{c}$. We can draw the parallel between a damped simple 


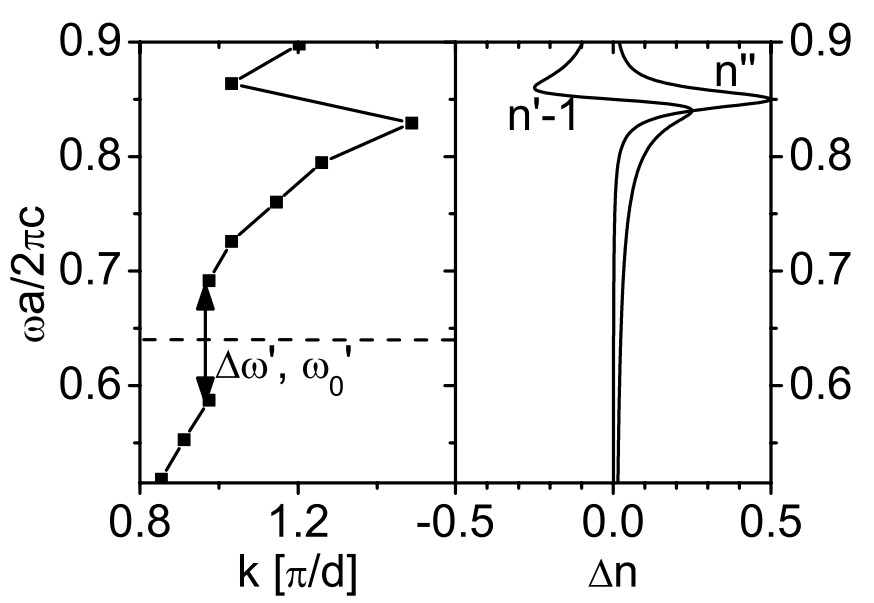

Figure 1.5.: (a) Schematic dispersion relation in the presence of a photonic vapor, whose resonance is just blue of the stopgap. At the red edge of the resonance, $n^{\prime}$ has increased above unity (see (b)), and the dispersion relation gives a higher $k$ for a given $\omega$. At the blue edge of the resonance, $n^{\prime}$ is below unity and $k$ is lower. (b) Material complex refractive index $n$ of an atomic vapor, whose resonance is at higher frequencies than the stopgap.

harmonic oscillator (SHO) and the cavity: both can store energy, and the energy leakage is inversely proportional to $Q$. The storage of energy results from the resonant recirculation, which is sustained for a time $\tau_{\text {cav }}=Q / \omega_{c}$, the cavity dwell time. The existing mode implies perfect transmission at the cavity resonance $\omega_{c}$, or vanishing reflectance. The delay caused by recirculation, and the frequency dependent transmission can be expressed in terms of the change in structural refractive index, see figure 1.6(b). At resonance, $n^{\prime \prime}$ vanishes, implying a high transmission. The real part $n^{\prime}$ has a large derivative $d n^{\prime} / d \omega$, implying a low group velocity $d \omega / d k=c /(n+\omega d n / d \omega)$, or a long pulse delay. We conclude that the inclusion of a defect in a photonic crystal influences the spectral and temporal response of the entire crystal.

\subsection{This thesis}

In this thesis, we experimentally demonstrate effects of all three resonances in various photonic crystals.

- In Chapter 2, we present the experimental setup that was used to demonstrate all-optical switching of microcavities and woodpile pho- 


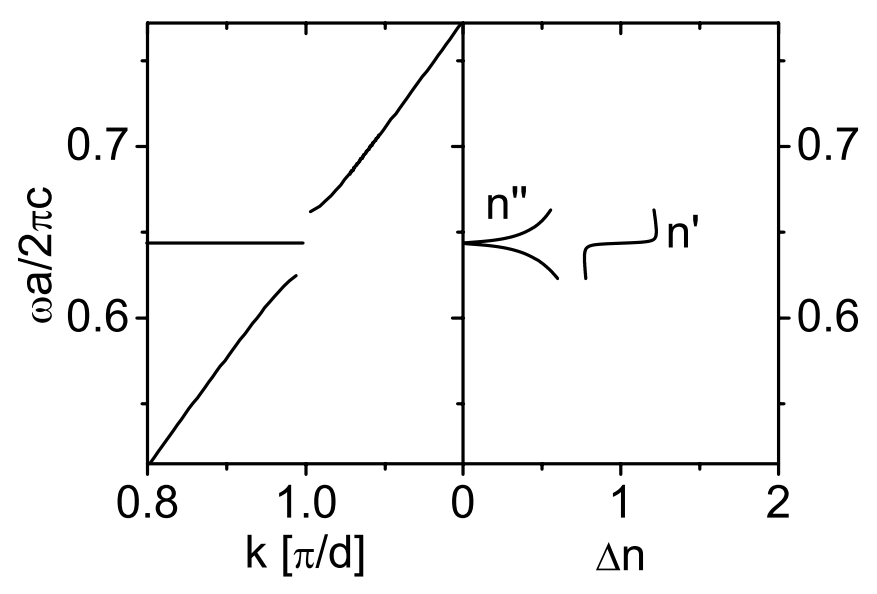

Figure 1.6.: (a) Schematic dispersion relation of a cavity in a photonic crystal. The cavity creates a mode in the stopgap of the dispersion relation. (b) The change in the structural complex real and imaginary index to that of a bare photonic crystal. The complex refractive index has been calculated for a finite Fabry-Pérot étalon from a relation given by [94].

tonic crystals. Although short, Chapter 2 is a prerequisite in comprehending our results presented in Chapters 3-5.

- Chapter 3 contains an experimental study of ultrafast all-optical switching of a microcavity resonance by several linewidths by free carrier excitation. We are able to infer the dynamic cavity resonance from broadband time-resolved measurements, and interpret the dynamic cavity resonance from the extended Drude model. Frequency-resolved measurements give insight into broadening mechanisms.

- In Chapter 4, we investigate the behavior of a pulse that has been trapped in a cavity from frequency resolved measurement, and whose resonance changes upon incidence of a pump pulse. With a physically intuitive model, spectral features are elucidated.

- Research on switching 3D photonic bandgap crystals is performed in Chapter 5. We identify two different switching regimes, in picosecond and in femtosecond timescales. The former regime is caused by caused by free carriers and can be interpreted with a Drude model, the latter is analysed in terms of two competing instantaneous effects. These two 
effects have ramifications on non-degenerate switching homogeneity, and ultimately on instantaneous DOS switching.

- Chapter 6 describes first-ever measurements of an atomic vapor, of $\mathrm{Cs}$, in an opal. The high strength of the atomic transition is shown to strongly modify the opal's reflectivity. Results are interpreted in terms of a modified transfer-matrix model, taking into account both the dispersion and the absorption of the atoms. 


\section{Chapter 2}

\section{Experimental setup and alignment}

We describe an ultrafast two-color pump-probe setup [95; 96], which we use to study semiconductor photonic structures (Chapters 3-5). In particular, we show how background free differential reflectivity spectra can be measured. We discuss both time-resolved broadband and frequency resolved detection.

\subsection{Pump and probe beams}

Figure 2.1 shows a schematic representation of our experimental setup. It consists of a regeneratively amplified Ti:Saph laser (Spectra Physics Hurricane) which drives two independently tunable optical parametric amplifiers (OPAs, Topas) with a repetition rate of $\Omega_{\text {rep }}=1 \mathrm{kHz}$. The frequency of both OPAs are computer controlled and have a continuously tunable output frequency between 0.44 and $2.4 \mathrm{eV}$. If we assume a Gaussian profile, the pulse duration is $\tau_{P}=140 \pm 10$ fs (measured at $E_{\text {Pump }}=0.95 \mathrm{eV}$ ), ${ }^{1}$ and the spectral width $\Delta E / E_{0}=1.33 \%$ [98]. Transform limited pulses would have a duration of $\tau_{P}=110 \mathrm{fs}$, and thus the pulses are nearly transform limited. The delay stage is computer controlled and can introduce a path difference of $40 \mathrm{~cm}$ to the probe, corresponding to a time delay of $1.3 \mathrm{~ns}$, much longer than typical recombination times in laminar structures or polysilicon, two structures we perform measurements on. The resolution is $10 \mathrm{fs}$, and thus much higher than the pulse durations. The pump is focussed onto the sample under an angle of $\theta=15^{\circ}$ by an achromatic lens of $\mathrm{NA}=0.01$.

\footnotetext{
${ }^{1} \tau_{P}$ denotes the FWHM of the pulse intensity, see e.g. [97], and was measured in an autocorrelator.
} 


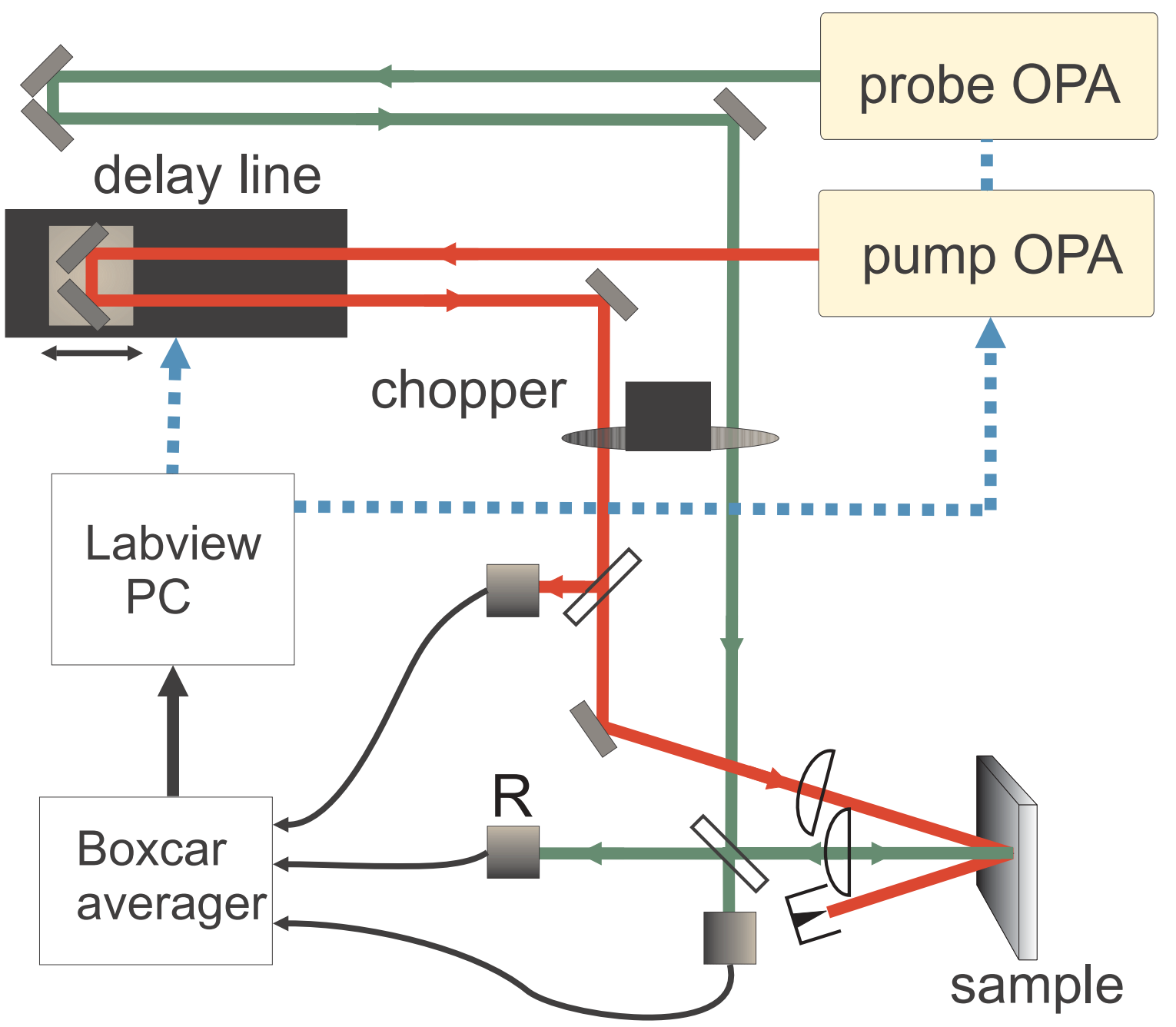

Figure 2.1.: Schematic drawing of setup. The pump and probe OPA's (TOPAS) are driven by a Hurricane (not shown) emitting 120 fs pulses. The pump pulses are delayed via a delay stage. After the pulses pass through a chopper, both probe and the pump pulses are separately monitored by diodes. The pulses are focussed onto the sample via achromatic lenses, and the irradiance of the reflected probe pulses are measured via an InGaAs diode $R$. The intensities of each monitored and reflected pulse is sampled and held by a boxcar averager, which offers the integrated irradiance to a $P C$, which stores every single pulse for later evaluation. In the frequency resolved setup, we replaced the diode $R$ with a spectrometer which we operated in free-running mode, and thus not connected to the boxcar. Figure lightly adapted from T. G. Euser [95]. 


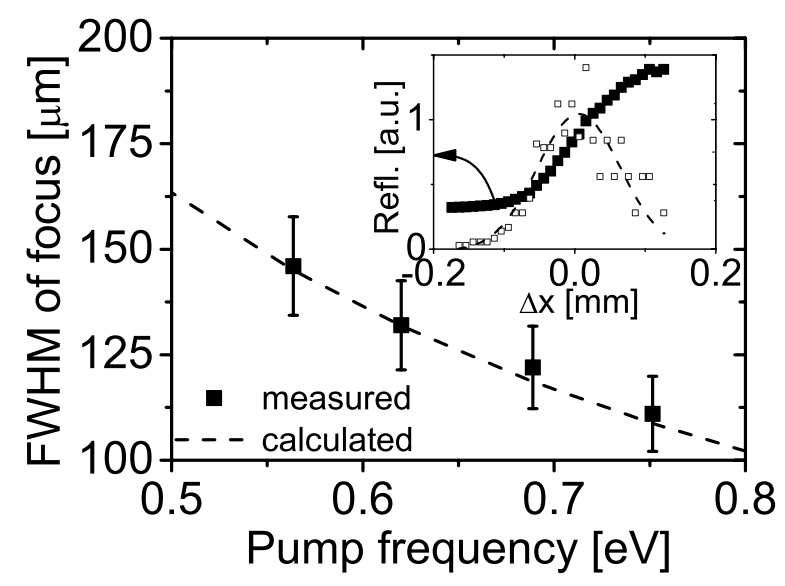

Figure 2.2: Measured pump beam focus diameters ( $2 r$, full width at half maximum, FWHM) vs.

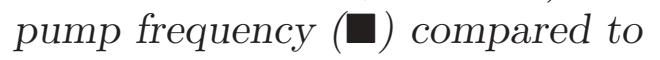
the diffraction limited focus size of a Gaussian beam (dashed curve). Inset: The diameters were determined by measuring the pump reflectance (shown for $E_{\mathrm{Pump}}=$ $0.62 \mathrm{eV}$ ) of a cleaved $\mathrm{Si}$ wafer scanned through the focus ( $\mathbf{\square}$, left scale) and fitting the derivative ( $\square$, right scale) to a Gaussian (dashed curve, right scale).

The peak irradiance for a focussed Gaussian pulse is given by

$$
I_{\text {pump }}=\frac{4 \sqrt{\ln 2} G}{\pi^{\frac{3}{2}} r^{2} \tau_{P}},
$$

where $r$ is the irradiance radius in the waist, and $G$ the energy of the pulse. Because $r$ depends on the pump frequency $E_{\text {pump }}$, the irradiance will depend on pump frequency. It is therefore important to measure the pump radius, so that the irradiance can be derived. In figure 2.2, we show the measured pump diameters $2 r$ at the focus for several pump frequencies. The diameters were obtained by measuring the reflected irradiance of the pump beam as a sharp-edged Si wafer is scanned through the focus (see inset). The reflected irradiance is the integral of the light distribution in the focus, and is an error function for a Gaussian beam. We therefore fitted the derivative of the measured irradiance to a Gaussian, from which the widths are readily obtained (see inset). The resulting diameters are compared to the diffraction limited diameter under an angle of $\theta=15^{\circ}$, and excellent agreement is obtained.

The probe beam is normally incident $\theta=0^{\circ}$ on the sample, and is focused to a Gaussian spot of $32 \mu \mathrm{m}$ FWHM (at $E_{\text {probe }}=1.24 \mathrm{eV}$ ) at a small angular divergence $\mathrm{NA}=0.02$. Because of the smaller probe focus with respect to that of the pump, only the flat part of the pump focus is probed, resulting in good lateral homogeneity. The reflectivity was calibrated by referencing to a gold mirror. To avoid carrier generation by the probe, we verified during all experiments that the probe pulses on the sample were ten times less intense than the pump pulses. 


\subsection{Broadband detection}

We have performed two sets of experiments that differ through their detection schemes. In chapters 3-5 we will elaborate which of the measurement schemes best fits the relevant purpose. In the first scheme, we measured both the reflected probe as well as the pump and probe irradiance monitors with InGaAs diode detectors. To reduce the noise caused by the low probe powers, and the possible background caused by scattered pump light, a versatile measurement scheme was developed to subtract the pump background from the probe signal, and to compensate for possible pulse-to-pulse variations in the output of our laser $[95 ; 96]$. The pump and probe beams were aligned in the same horizontal plane, but mirrored around the rotation axis of the chopper, see figure 2.3. The chopper was synchronized to the repetition rate $\Omega_{\text {rep }}$ of the Hurricane, but the rotation speed was so that only two consecutive pump or probe pulses may pass the chopper, while the following two pump or probe pulses are blocked. Because additionally the horizontal position of the beams is mirrored by the chopper axis, the train of pump and probe pulses is phase shifted by $\pi$. At the focus, four permutations of pump and probe beam occur: In (a), both the pump and the probe pulse pass the chopper. A time $1 / \Omega_{\text {rep }}$ later, the chopper blades block the pump pulse, but pass the probe pulse (b). No pulse may pass in (c) (opposite to (a)), while (d) is the opposite to (b): only the probe passes. The linear (unpumped) reflectance is given by $R^{\mathrm{up}}=J^{\mathrm{up}}-J_{\mathrm{bg}}^{\mathrm{up}}$, where $J^{\text {up }}$ is the detector signal at R when the chopper is in position (d), while $J_{\mathrm{bg}}^{\mathrm{up}}$ is the signal at $\mathrm{R}$ at chopper position (c). To compensate probe pulse fluctuations, $R^{\text {up }}$ is then ratioed by the background-corrected probe monitor signals $M^{\text {up }}$, measured when the chopper is at position (d) and (c). In a similar manner, the non-linear (pumped) reflectance can be determined to be $R^{\mathrm{p}}=J^{\mathrm{p}}-J_{\mathrm{bg}}^{\mathrm{p}}$, where $J^{\mathrm{p}}$ and $J_{\mathrm{bg}}^{\mathrm{p}}$ are the signals measured on $\mathrm{R}$ at chopper positions (a) and (b), respectively, and is also ratioed by the corresponding probe monitor signals. This process obviously requires the three detectors to store all four signals during $4 / \Omega_{\text {rep }}$. When this happens, the differential reflectivity $\Delta R / R$ corrected for background and fluctuations can thus be determined by

$$
\frac{\Delta R}{R} \equiv \frac{R^{\mathrm{p}} / M^{\mathrm{p}}-R^{\mathrm{up}} / M^{\mathrm{up}}}{R^{\mathrm{up}} / M^{\mathrm{up}}} .
$$

To further increase the signal to noise ratio, we typically average over 1000 pulses, or 250 data points. In order to more precisely analyse data, all signals from the three detectors are stored while measuring. Before measuring 

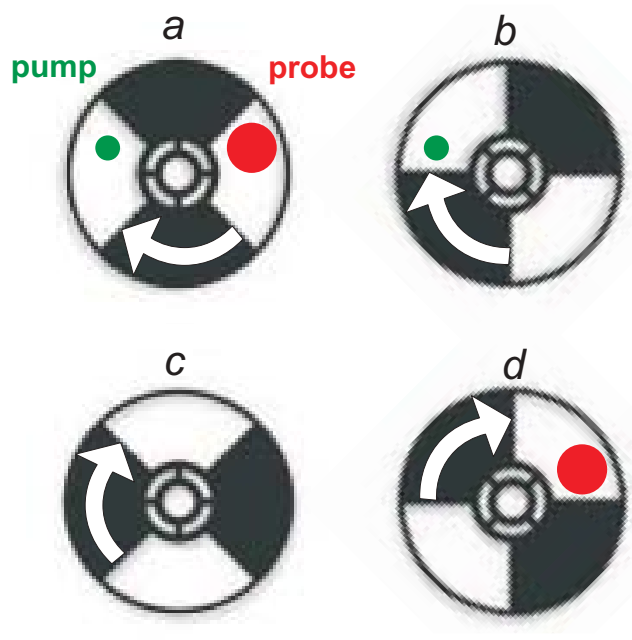

Figure 2.3: Detection scheme of the broadband detection: both the pump and the probe pulses are synchronized to the chopper, and arrive simultaneously. The rotation rate is a quarter of the repetition rate. Then, all four permutations of pump or probe are incident on the sample. (a) Both pump and probe pass the chopper. (b) $1 / \Omega_{\text {rep }}$ later, the chopper blades block the probe pulse, but pass the pump pulse. (c) Both pump and probe are blocked. (d) Only the probe passes. Figure courtesy of T. G. Euser.

the differential reflectivity, we separately measured the reflectance of a gold mirror for all probe frequencies, and verified that the reflectivity obtained agreed well to that measured independently with a cw setup.

We measured both the reflected probe as well as the pump and probe irradiance monitors with InGaAs diode detectors. A boxcar averager, synchronized to the pulse trigger, integrates and holds the detected signal before being read out by a digital to analogue converter (DAC). The signal $J$ offered to the DAC card by the boxcar, neglecting electronic amplification factors, is equal to the magnitude of the time- and space integrated Poynting vector S,

$$
\begin{aligned}
J=\pi R^{2} \int_{-t_{\mathrm{int}} / 2}^{t_{\mathrm{int}} / 2}|\mathbf{S}| d t & =\int_{-t_{\mathrm{int}} / 2}^{t_{\mathrm{int}} / 2} \sqrt{\frac{\epsilon_{0}}{\mu_{0}}} \digamma(t)^{2} d t \\
& \approx \pi R^{2} \sqrt{\frac{\epsilon_{0}}{\mu_{0}}} \frac{\tilde{\digamma}_{0}^{2}}{2} \int_{-\infty}^{\infty}\left(\exp \left(-4 \ln 2 t^{2} / \tau_{G}^{2}\right)\right)^{2} d t \\
& =\pi R^{2} \sqrt{\frac{\epsilon_{0}}{\mu_{0}}} \sqrt{\frac{\pi}{2 \ln (2)}} \frac{\tau_{P} \tilde{\boldsymbol{\digamma}}_{0}^{2}}{4}
\end{aligned}
$$

where the electric field $\digamma(t)$ reflected by a perfect mirror onto the detector can be separated in a Gaussian envelope $\tilde{\boldsymbol{F}}(t)$ of FWHM $\tau_{G}$ and amplitude $\tilde{\digamma}_{0}$ multiplied by an sinusoidal component with a carrier frequency $\omega_{0}$ in $\mathrm{rad} / \mathrm{s}^{2}$

${ }^{2}$ This Slowly Varying Envelope Approximation (SVEA, see, e.g. [97]) can be applied to pulses where $\tau_{P}>1 / \omega_{0}$, and where $\omega_{0}$ does not change over $\tau_{P}$, i.e., for bandwidth limited pulses. For pulses whose envelope is broadened by interaction with a cavity, 
The squared oscillating term can be then integrated separately and yields $1 / 2$, and the time integration can be taken to infinity because $t_{\text {int }}>>\tau_{P}$. Since the integration time of the boxcar $\left(t_{\text {int }} \sim 150 \mathrm{~ns}\right)$ is much longer than any probe interaction time, ${ }^{3}$ the dynamics of the sample is essentially integrated over. The beam is collimated and has radius $R$. $\epsilon_{0}$ and $\mu_{0}$ denote the permittivity and permeability of free space, respectively.

\subsection{Frequency-resolved detection}

In a second set of experiments, we made use of the large probe bandwidth and we resolved spectral features with a spectrometer. Theses narrow spectral features occurred in the microcavity samples discussed in Chapters 3 and 4, see, e.g, figure 3.5. We accomplish this with a spectrograph PI/Acton SP-2558, using a 1024 channel InGaAs line detector (OMA-V), yielding a resolution of $0.12 \mathrm{meV}$ at $1.24 \mathrm{eV}$. The diode array is kept at a temperature of $100 \mathrm{~K}$ to reduce dark counts, measured to be $350 \mathrm{adu} /(\mathrm{s}$ pixel)), which is then only $1 \%$ of the counts due to probe pulses of several nJ. The dynamic reflectivity was determined by referencing the reflectance to a gold mirror. Even though the effective repetition period $\left(\Omega_{\mathrm{rep}} / 2=1 / 500 \mathrm{~Hz}\right)$ of the laser is equal to the minimum exposure time of the detector electronics (2 $\mathrm{ms})$, the OMA-V was operated in free running mode, with an integration time set to $1 \mathrm{~s}$, as no additional useful information was expected in single shot measurements. The measured spectra thus consist of $1 \mathrm{~s} \cdot 500 \mathrm{~Hz}=500$ pulses. The observed spectrum, again without amplification and conversion factors, is a Fourier Transform of $\digamma(t)$ :

$$
J(\omega)=\pi R^{2}\left(\epsilon_{0} c\right)^{-1}\left|\int_{-\infty}^{\infty} d t \digamma(t) e^{i \omega t}\right|^{2}
$$

where $c$ is the celerity of light in free space. A pulse travelling in a medium of changing refractive index can attain frequency components whose amplitude are higher than when the pulse commenced its travel. In that case, the ratio of the reflected pulse to a reference pulse, the transient reflectivity, $J(\omega)_{\text {sample }} / J(\omega)_{\text {ref }}$ may exceed unity for some $E_{\text {Probe }}$.

the analytic expression obtained (eq. 2.3) is not valid, but the approximation of the integration limits does not change.

${ }^{3}$ The probe interaction time is either $\tau_{P}$ or $Q / \omega_{0}$, whichever is greater, and is in the 100 fs to 1 ps range. 


\section{Dynamical ultrafast all-optical switching of planar GaAs/AIAs photonic microcavities}

\subsection{Introduction}

There is generally a great interest to store photons in a small volume. This feat can be achieved in solid state structures with tiny cavities, with dimensions of the order of the wavelength of light. Light is so strongly confined in such cavities that large electric field enhancements occur. This field enhancement notably leads to large modifications of the emission rate of an elementary light source embedded inside a cavity [91; 99]. It is highly desirable, both from fundamental and applied viewpoints, to switch the optical properties of cavities on ultrafast time scales $[66 ; 100 ; 101]$. This ultrafast switching of cavities will allow the catching or releasing of photons, changing the frequency and bandwidth of confined photons, and even the switchingon or -off of light sources $[83 ; 84 ; 86 ; 102 ; 103]$. It is therefore important to systematically study the dynamic behavior of switched cavities. Surprisingly, such studies are scarce. Recently, Almeida et al. studied relaxation at two frequencies for a large 10 micron diameter Si ring resonator, revealing decay times of $0.45 \mathrm{~ns}$ [81]. Here, we use broadband tunable femtosecond pump-probe reflectivity to study the dynamics of planar thin $\lambda$-microcavities made from III-V semiconductors, an important class of solid-state cavities that are notably used in vertical-cavity surface-emitting lasers [76].

\subsection{Experimental setup: sample and linear reflectivity}

The experimental setup has been described in Chapter 2. Our sample consists of a GaAs $\lambda$-cavity with a thickness of $275.1 \pm 0.1 \mathrm{~nm}$. The layer is 
sandwiched between two Bragg stacks consisting of 12 and 16 pairs of $\lambda / 4$ thick layers of nominally pure GaAs or AlAs. The microcavity is supported by GaAs substrate of thickness $200 \pm 20 \mu \mathrm{m}$. The sample, made by Y.-R. Nowicki-Bringuier using the molecular beam epitaxy facilities of the CEA, Grenoble, is grown at $550^{\circ} \mathrm{C}$ to optimize the optical quality (see section 3.3.1). For experiments outside the present scope the sample was doped with $10^{10} \mathrm{~cm}^{-2}$ InGaAs/GaAs quantum dots, which hardly influence our experiment. ${ }^{1}$ The sample's dimensions were $x \cdot y=11 \mathrm{~mm} \cdot 2 \mathrm{~mm}$. Continuouswave $(\mathrm{cw})$ reflectivity was measured with a Fourier-Transform spectrometer (BioRad FTS-6000, resolution $0.25 \mathrm{meV}$ ) and a supercontinuum white light source (Fianium) [54] focussed onto the sample with a microscope objective $\mathrm{NA}=0.12$.

\subsection{Results: dynamic cavity resonance}

\subsubsection{Linear reflectivity}

Figure 3.1 shows a cw reflectivity spectrum of the planar photonic microcavity at normal incidence. The high peak between 1.192 and $1.376 \mathrm{eV}$ is due to the stopgap of the Bragg stacks. The stopband has a broad width $\Delta \mathrm{E}$ $=184 \mathrm{meV}$, or $14.3 \%$ relative bandwidth, which confirms the high photonic strength. At both the blue and the red side of the stopgap we observe FabryPérot fringes. The Fabry-Pérot fringe at $1.15 \mathrm{eV}$ exceeding $100 \%$ is due to some chromatic abberation in the focus. Near $E_{\text {cav }}=1.279 \mathrm{eV}$ we observe a sharp resonance caused by the $\lambda$-cavity in the structure (see inset): when the cavity thickness is $K \lambda / 2$ ( $\mathrm{K}$ is an integer), the cavity acts as a Fabry-Pérot étalon and a standing wave of wavelength $\lambda=h c / n^{\prime} E_{\text {Probe }}$ forms in the cavity [107] (Planck's constant is $h$ and the real part of the refractive index is $n^{\prime}$ ). The resonance has a linewidth $\Delta_{w, 0}^{\text {meas }}=1.7 \mathrm{meV}$, corresponding to a quality factor $Q_{w, 0}^{\text {meas }}=750 .^{2}$ A transfer matrix $(\mathrm{TM})$ calculation including the dis-

\footnotetext{
${ }^{1}$ The maximum unsaturated unbroadened refractive index change of the dots amounts to only $10^{-8}$, while the absorption at resonance is less than $50 \mathrm{~cm}^{-1}$. Here, we modeled the QDs as Lorentz oscillators with an oscillator strength of $f=10$, a height of $7 \mathrm{~nm}$ [104], and a FWHM width at $300 \mathrm{~K}$ of $\Gamma=10 \mathrm{meV}$ [105]. Interband absorption was comparable to our calculated intraband absorption for our probe frequencies [106].

${ }^{2}$ The definition of the quality factor $Q$ of a damped oscillator is $2 \pi$ (Energy stored)/(Energy loss per cycle). $E_{\text {cav }} / Q$ is the FWHM of its intensity frequency response, and also the intensity decay constant. The spectral width of the reflectivity trough of a microcavity is related to $E_{\text {cav }} / Q$, but not necessarily equal to it. In the following we will denote observables derived from the width of the reflectivity by the
} 
persion and absorption [108; 109] of GaAs and the dispersion of AlAs [110] reproduces the experimental resonance, stopband, and Fabry-Pérot fringes well. The only free parameters in the model were the thicknesses of the $\operatorname{GaAs}\left(d_{\mathrm{GaAs}}=68.78 \pm 0.03 \mathrm{~nm}\right)$ and AlAs $\left(d_{\mathrm{AlAs}}=81.90 \pm 0.03 \mathrm{~nm}\right)$. The $Q$ calculated from the TM model $Q_{w, 0}^{\text {calc }}$ is 1714 , which is more than twice the measured value. The main reasons for the discrepancy are spatially varying resonances and wavevector spreading due to a finite NA (see below).

For different positions on the sample, both the resonance and the width changed, while the relative width remained constant. The cavity resonance varied smoothly in $x$-direction over $50 \mathrm{meV}$, with a gradient of $d E_{\text {cav }} / d x=$ $m=4.55 \mathrm{meV} / \mathrm{mm}$. The lineshape due to spatially varying resonances is different for every position. Thus, this lineshape is inhomogeneously broadened. Homogeneous broadening results from wavevector spreading, and is the same for every sample position. The inhomogeneous lineshape $I_{\text {inhom }}$ is given by

$$
I_{\text {inhom }}(E)=\frac{\int_{0}^{r} \int_{-\pi / 2}^{\pi / 2} d \phi d R I_{\text {hom }}\left(E-E_{\text {cav }}(\phi, R)\right) r^{2} \cos ^{2}(\phi) e^{-4 \ln (2) \frac{R^{2}}{r^{2}}}}{\int_{0}^{r} \int_{-\pi / 2}^{\pi / 2} d \phi d R r^{2} \cos ^{2}(\theta) e^{-4 \ln (2) \frac{R^{2}}{r^{2}}}},
$$

and

$$
\lim _{a \rightarrow 0} I_{\mathrm{hom}}(E)=\frac{\int_{a}^{\phi_{\max }} d \theta L\left(E-E^{\prime}, \Delta\right) e^{-4 \ln (2) \frac{\tan ^{2}(\theta)}{\tan ^{2}\left(\theta_{\max }\right)}} \tan (\theta) \sec ^{2}(\theta)}{\int_{a}^{\theta_{\max }} d \theta e^{-4 \ln (2) \frac{\tan ^{2}(\theta)}{\tan ^{2}\left(\theta_{\max }\right)}} \tan (\theta) \sec ^{2}(\theta)} .
$$

Here, the homogeneous lineshape $I_{\text {hom }}$ depends on three factors: a., the angle dependent intrinsic Lorentzian resonance $\left.L\left(E-E^{\prime}(\theta)\right), \Delta\right)$ of FWHM width $\Delta$ and resonance $E^{\prime}(\theta)=E_{\text {cav }} / \cos (\theta)$, b. the maximum angle $\theta_{\text {max }}$ subtended by the focussed beam at the focus, and c. the intensity distribution of the probe beam at the lens. This intensity distribution is modeled as a Gaussian of FWHM $\tan \left(\theta_{\max }\right)$. Only a small part of the intensity distribution is focussed through the lens as the beam has been collimated by a diaphragm. The inhomogeneous lineshape is the homogeneous lineshape integrated over the focus of radius $r$. The integration is over radius $R$ and azimuthal angle $\phi$, and is weighted by the intensity distribution in the focus, assumed to be a Gaussian of FWHM $r$.

subscript $w$, unswitched by the subscript 0 , and measured/calculated with the superscript meas/calc, respectively. 


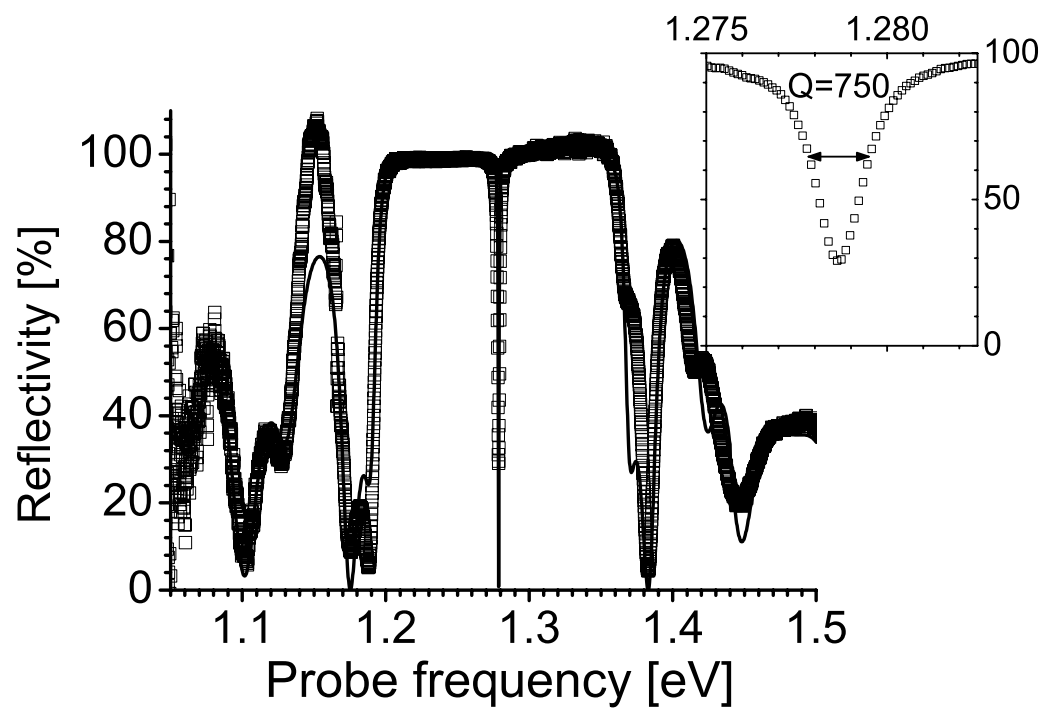

Figure 3.1.: Continuous wave reflectivity spectrum (open circles) at normal incidence of sample with a resolution of $0.25 \mathrm{meV}$. The resonance of the $\lambda$-cavity $\left(Q_{w, 0}^{\text {meas }}=\right.$ $750)$ can clearly be seen at $E_{\text {cav }}=1.279 \mathrm{eV}$ (see inset). The solid curve is a transfer matrix calculation that includes the dispersion and absorption of GaAs and AlAs.

For $\Delta=E_{\text {cav }} / Q_{0}^{\text {calc }}$ and an external $\mathrm{NA}=0.12$ (internal $\mathrm{NA}=0.12 / n_{\text {eff }}=$ 0.038 ), the width from $I_{\text {hom }}$ is $2 \mathrm{meV}$, or a $Q=621$. Inhomogeneous broadening yields $Q=606$, reasonably close to $Q_{w, 0}^{\text {meas }}=750$. Inhomogeneous broadening is thus negligible for the estimated beam radius of $r=50 \mu \mathrm{m}$ and for high NA. For an experiment NA $=0.02 / n_{\text {eff }}$, the $Q_{w}$ from homogeneous width is $Q=1612$, while the inhomogeneous broadening $Q_{w}=1389$, also close to $Q_{w, 0}^{\text {meas }}=1242$ (fig. 3.5). For this configuration, the spatial distribution of resonances is much more important, just as for larger focus radii (e.g. $\mathrm{r}=0.1 \mathrm{~cm}$ ): the angular spread of wavevectors is negligible, $Q_{w}$ yielding 135 and 133 for $\mathrm{NA}=0.02 / n_{\text {eff }}$ and $\mathrm{NA}=0.12 / n_{\text {eff }}$, respectively. We conclude that our model gives a good estimation of the observed linewidths, and that therefore the different linewidths observed in the two different setups are due to a combination of wavevector spreading and, to a lesser extent, spatially changing resonance conditions. Because $Q_{w, 0}^{\text {meas }}$ depends on these two factors, in the following all relative changes will be stated with respect to $Q_{w, 0}^{\text {meas }}=1242$. 


\subsubsection{Dynamic reflectivity}

We dynamically probe the excited cavity by time-resolved pump-probe reflectivity. The consistency of our data is verified in 5 experimental runs. Near pump and probe coincidence (i.e, probe delay $\Delta t=0 \mathrm{fs}$ ), the differential reflectivity at the unswitched cavity resonance briefly decreases during an interval $\Delta \tau_{0}=218 \pm 5$ fs full width at half minimum (FWHM), see figure 3.2a. This value agrees well with $\sqrt{2} \tau_{\mathrm{P}}=200 \pm 15 \mathrm{fs}$ for the crosscorrelation of the pump and probe pulses, which signals an instantaneous non-linear process. Figure 3.2a shows an increase of reflectivity at longer probe delays. This is the result of the excited free carriers $[111 ; 112]$ that decrease the index and thereby blueshift the cavity resonance. After about $50 \mathrm{ps}$, the changes in differential reflectivity have nearly vanished due to the recombination of the free carriers. Decay constants for probe frequencies much outside the cavity resonance yielded a factor $\sim 2$ shorter decay times. While other authors have limited their studies to either the reflectivity at two frequencies $[66 ; 78-82 ; 113]$ or to the reflectivity at two probe delays [102], we probe at all frequencies and delays which allows us to extract the dynamic cavity resonance, and to assess effects of dynamic broadening.

To dynamically track the cavity resonance, we have measured the timeresolved differential reflectivity for a large spectral range, see fig. 3.2b. The data clearly demonstrate the free carrier induced blue shift: the differential reflectivity increases at high frequencies and decreases at low frequencies. From the data we have extracted the time-dependent cavity resonance. ${ }^{3}$ Between 0 and $\tau_{\mathrm{ON}}=6 \mathrm{ps}$, the cavity resonance quickly shifts to higher frequencies. The maximum shift is $\Delta E_{\text {cav }}=4.8 \mathrm{meV}$, corresponding to 4.7 times the unswitched linewidth $\Delta_{0}^{\text {meas }}$ (cf. figure 3.1). Subsequently, the frequency of the resonance returns to the unswitched case with a time

${ }^{3}$ We have derived the wavelength of the cavity resonance by using the identity

$$
\frac{d(\Delta R / R)}{d \nu}=\frac{1}{R_{0}(\nu)^{2}}\left(R_{0}(\nu) \frac{d R(\Delta t, \nu)}{d \nu}-R(\Delta t, \nu) \frac{d R_{0}(\nu)}{d \nu}\right)
$$

where $R_{0}(\nu)$ is the unswitched reflectivity, $R(\Delta t, \nu)$ is the switched reflectivity at delay $\Delta t$, and $\nu$ is a shorthand for $E_{\text {Probe }}$. Setting $\frac{d R(\Delta t, \nu)}{d \nu}=0$, which is the necessary extremal condition for the cavity resonance, we obtain

$$
0=\frac{d\left(\Delta R(\Delta t, \nu) / R_{0}(\nu)\right)}{d \nu}+\frac{R(\Delta t, \nu)}{R_{0}(\nu)^{2}} \frac{d R_{0}(\nu)}{d \nu} .
$$

Thus, the dynamic resonance wavelength is completely determined by the experimental data. 


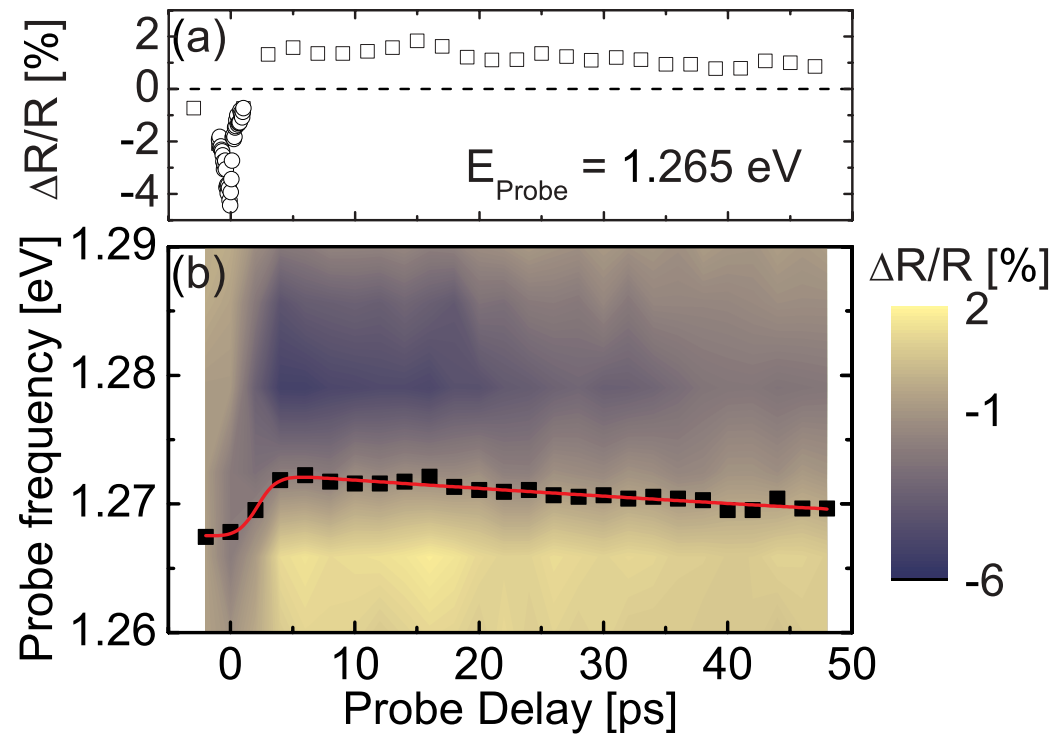

Figure 3.2.: (a) Differential reflectivity versus probe delay at a wavelength close to the cavity resonance measured at a different sample position than the $\mathrm{cw}$ reflectivity (figure 3.1). The time resolution is $2 \mathrm{ps}$. Close to $\Delta t=0 \mathrm{ps}$ we took an additional scan at high time resolution (30 fs), which shows a trough with a width of $\sim \sqrt{2} \tau_{P}$ around $\Delta t=0 \mathrm{fs}$, indicative of instantaneous probe absorption. (b) Differential reflectivity versus probe delay and frequency (resolution: $6.4 \mathrm{meV}$ ). At $\Delta t>100$ fs, $\Delta R / R$ increases (decreases) at the blue (red) edge of the cavity, indicating a blue shift of the stopband and resonance. The extracted cavity resonances ( $\mathbf{\square})$ are connected by a guide to the eye (solid curve). The pump and probe intensities are $I_{\text {Pump }}=180 \pm 18 \mathrm{GWcm}^{-2}$ and $I_{\text {Probe }}=7 \pm 2 \mathrm{GWcm}^{-2}$, respectively.

constant $\tau_{\mathrm{OFF}}$ of $57 \pm 2$ ps.

To investigate the dynamic behavior of the cavity in more detail, we plot in figure 3.3 the differential reflectivity versus frequency at selected delays. When the probe pulse arrives before the pump pulse $(\Delta t=-2 \mathrm{ps}), \Delta R / R$ is slightly negative, but shows some spectral features. Since the probe pulse takes a time $2 d n_{\mathrm{eff}} / c=2 \cdot 200 \cdot 10^{-6} \mathrm{~m} \cdot n_{\mathrm{GaAs}} / c=4.7$ ps to pass through the sample twice, the probe meets the incoming pump pulse on reflection from the rear side of the substrate [95]. The bandwidth of the probe however now matches the linewidth of the cold cavity. Since the cavity resonance has now shifted with respect to the unswitched cavity resonance, only the blue wing of the probe spectrum may pass through the microcavity. A model taking into account the probe spectrum, the switched and unswitched cavity resonance 


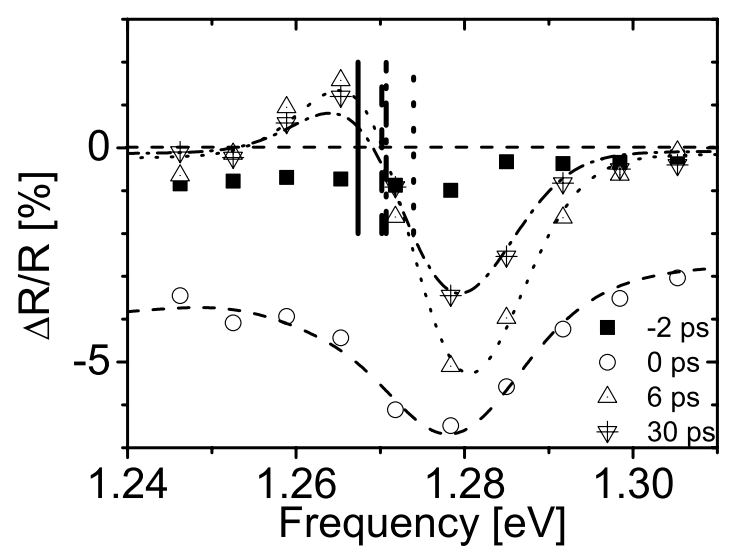

Figure 3.3.: Differential reflectivity versus probe frequency for selected probe delays. The curves are transfer matrix calculations, the vertical bars indicate the frequencies of the cavity resonance.

and linewidth excellently reproduces the observed features. However, all combinations of free carrier absorption and rear reflection coefficient yielded a differential reflectivity spectrum whose magnitude was two orders higher than the observed one, and whose spectral position was red shifted by 15 meV with respect to the measured spectrum (not shown). From this analysis we conclude that the dynamics of a narrowband probe pulse being subjected to a rapidly changing refractive index may be more subtle than assumed. At pump and probe coincidence, the differential reflectivity has decreased and reveals a broad minimum. The decreased reflectivity is attributed to nondegenerate two-photon absorption, since the sum of the pump and probe frequency $E_{\text {Total }}=1.99 \mathrm{eV}$ is much above the optical bandgap of GaAs $(1.44 \mathrm{eV})$. At $\Delta t>0 \mathrm{ps}$, the differential reflectivity acquires a dispersive shape, typical for the shift of a resonance. Until $\Delta t=6 \mathrm{ps}$, the amplitude of the dispersive differential reflectivity increases in magnitude, due to the cavity's resonance shift, indicated by the bars in figure 3.3. By interpreting the measured differential reflectivity at 6 ps with a TM calculation that includes an extended Drude model to account for the excited carriers (see below), we obtain a carrier density of $N=1.22 \times 10^{19} \mathrm{~cm}^{-3}$.

\footnotetext{
${ }^{4}$ From this density we infer a degenerate two-photon absorption coefficient of $\beta=0.43 \pm$
} $0.09 \mathrm{cmGW}^{-1}$. 


\subsubsection{Dynamic carrier density and refractive index}

A quantitative analysis requires a mapping from the dynamic complex refractive index $n$ to $N$. For free electrons, this mapping is provided by the well-known Drude model [68]. Here, difference in $n$ due to free carrier dispersion can be approximated as

$$
\Delta n=\Delta n^{\prime}+\Delta i n^{\prime \prime}=-\frac{\omega_{P}^{2}}{2 n_{0} \omega_{0}^{2}}+i \frac{\omega_{P}^{2}}{2 \omega_{0}^{3} \tau_{D} n^{\prime}},
$$

where $\omega_{P}^{2}=N e^{2} / m_{e} \epsilon_{0}$ is the plasma frequency squared in $(\mathrm{rad} / \mathrm{s})^{2}$ and $e$ and $m_{e}$ are the charge and mass of an electron, $n_{0}=n_{\mathrm{GaAs}}$, and $\tau_{D}$ the Drude damping time. Equation 3.2 is valid for $\left(\omega \tau_{D}\right)^{-1}<<1$, which is the case for our probe frequencies and damping times.

However, the Drude model must be extended to correct for several limitations. First, since we excite both electrons and holes, both their masses in the energy bands must be included in the model. The mass $m_{e}$ is thus replaced with their effective optical mass $m^{*} .5$ Second, the electrons are not amenable to polarization within dephasing time $T_{2}$, and so will not exhibit Drude dispersion. Moreover, the electrons will first relax within the conduction band by emission of LO-phonons. During this time we clearly see a dynamic blue shift of the cavity resonance. Using the Drude model before $\tau_{\mathrm{On}}=6 \mathrm{ps}$ is therefore unphysical, where $\tau_{\text {On }}$ compares favorably to literature $[72 ; 76]$. Third, as soon as the carriers have dephased and thermalized, the relation between $N$ and $n^{\prime \prime}$ is essentially governed by a constant momentum relaxation time, the Drude damping time $\tau_{D}$, which is the mean free time before an elastic collision. For low carrier densities $\left(N \leqslant 10^{22} \mathrm{~m}^{-3}\right)$ this assumption is correct, as electrons scatter with phonons at a rate $\Gamma_{\mathrm{e}-\mathrm{ph}} \sim 1 / 250$ fs [115] (see below). At higher densities, the trajectory of carriers is additionally influenced by the Coulomb field of others, and the scattering rate $\Gamma_{c c}=\gamma N^{1 / D}, D$ being the dimensionality of the system $[70 ; 116]$. We found best agreement for a proportionality constant $\gamma=10^{5} \mathrm{~m} / \mathrm{s}$, which is between the values quoted by [70] and [117] obtained for bulk GaAs. Finally, while the Drude model accounts for the absorption due to excited electron transitions within one conduction band (intraband transitions), it does not account for transitions between bands (interband transitions). From [118], we extrapolated the corresponding absorption cross-section to be $5.6 \cdot 10^{-23}$ $\mathrm{m}^{2}$

\footnotetext{
${ }^{5}$ The effective optical mass $m^{*}=m_{e}^{*} m_{h}^{*} /\left(m_{e}^{*}+m_{h}^{*}\right)$, where the superscript ${ }^{*}$ denotes the mass in the bandstructure. Values from [114]
} 
We fitted the differential reflectivity from the TM model including the extended Drude model to our measured data for probe delays $\Delta t>6 \mathrm{ps}$ with one free parameter, $N$ (figure 3.4(a)). After the maximum density of $N=1.22 \times 10^{19} \mathrm{~cm}^{-3}$ at $\Delta t=6 \mathrm{ps}$, the carrier density decreases with an exponential time constant of $57 \pm 2$ ps due to recombination. This recombination time is an important parameter in switching applications, e.g. modulators, as it determines the maximum switching rate between state 'on' and 'off'. If we assume that the recombination has ceased at 100 ps, the switching rate is $1 / 100 \mathrm{ps}=10 \mathrm{GHz}$, one order of magnitude faster than previously reported [81], but comparable to [100], where ion implantation was used to reduce the recombination time in $\mathrm{Si}$, albeit at the cost of a degraded cavity linewidth. The maximum switching rates of microcavities may further be tenfold increased [119] by growing samples with a larger number of recombination centers at the GaAs/AlAs interfaces.

From the free carrier density $N$ and the extended Drude model, we have also calculated the time-dependent real (n') and imaginary (n") parts of the refractive index of the GaAs layers (figure 3.4(b) and (c)). The real part mostly determines the shift of the resonance wavelength, whereas the imaginary part allows to assess possible changes of the quality factor $Q_{w}$. The real part decreases by $\Delta n_{\mathrm{GaAs}}^{\prime}=-0.025 \pm 0.003$, or $0.7 \%$, corresponding to $4.5 \pm 0.5 \mathrm{meV}$, or $4.4 \pm 0.5$ linewidth shift, in agreement with the 4.7 linewidth shift determined previously. The imaginary part increases to $n_{\mathrm{GaAs}}^{\prime \prime}=0.71 \times 10^{-3}$ due to the free carriers, before returning to the unswitched value. From the maximum value of $n^{\prime \prime}$ at $6 \mathrm{ps}$, we estimate from a TM calculation that $Q_{w, 0}^{\text {calc }}$ is 0.6 times the unswitched cavity $Q$.

Near $\Delta t=0 \mathrm{fs}$, the imaginary index is briefly as large as $n^{\prime \prime}=1.6 \pm$ $0.3 \times 10^{-2}$, corresponding to a decrease of $Q_{w, 0}^{\text {calc }}$ to $7.7 \%$ of its original value. Here, $n^{\prime \prime}$ was obtained by fitting a TM calculation with a complex $n$ to the measured differential reflectivity (figure 3.3), and corresponds to a non-degenerate two-photon absorption coefficient for GaAs of $\beta_{12}=17 \pm 3$ $\mathrm{cmGW}^{-1}$, in agreement with $\beta_{12}=10 \mathrm{cmGW}^{-1}$ derived from ref. [120]. While this period of relatively high absorption lasts rather briefly, it is recommended to keep the sum of the probe and pump frequencies below the optical bandgap of the constituent materials or to reduce the pump fluence. In the next section, we will measure the linewidth dynamically. 


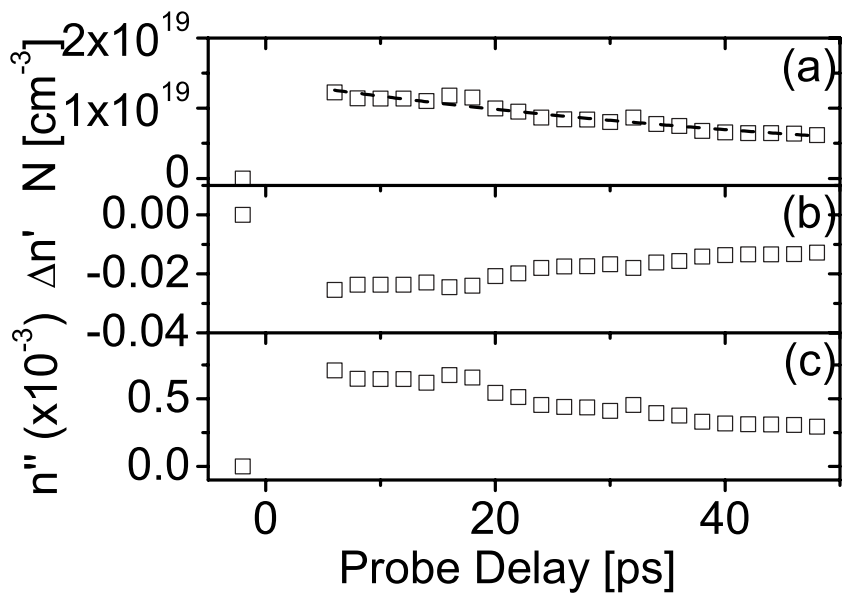

Figure 3.4.: (a) Carrier density versus probe delay as obtained from the differential reflectivity using the extended Drude model at delays of 6 ps and longer. We have fitted a single exponential (dashed curve) to the carrier density with $\tau_{\mathrm{OFF}}=57 \pm 2$ ps. (b) Change in real part n' and (c) imaginary part n" of the refractive index $n$ calculated with the extended Drude model and the carrier density.

\subsection{Results: Dynamic linewidth}

\subsubsection{Linear reflectivity}

The large probe bandwidth relative to the cavity linewidth offers the possibility of directly resolving both the switched as well as the unswitched cavity resonance. Figure 3.5 shows the reflected spectrum of the microcavity measured with our OPA, along with a reference spectrum. The Gaussian shaped reference has a width of $17.2 \mathrm{meV}$, or $1.35 \%$ relative width, in excellent agreement to the $1.33 \%$ measured previously [98]. The width of the cavity resonance is $\Delta_{0}^{\text {meas }}=1.03 \mathrm{meV}$ centered at $1.2783 \mathrm{eV}$, or $Q_{w, 0}^{\text {meas }}=1242$. Compared to our previous measurement (figure 3.1), the quality factor is nearly $50 \%$ higher. The seeming disagreement is partially because this setup had a lower $\mathrm{NA}=0.02$ compared to an $\mathrm{NA}=0.12$ for the cw reflectivity (cf. section 3.3.1). An improved beam collimation also agrees with the deeper transmission minimum of $12 \%$ with respect to $30 \%$ in figure 3.1 , explained by the reduced wavevector spreading.

A slight misorientation of the reference gold mirror caused a reference spectrum of around twice the expected magnitude, which, while it causes a possible decrease in modulation depth, does not affect the $Q_{w, 0}^{\text {meas }}$ [49]. Since 


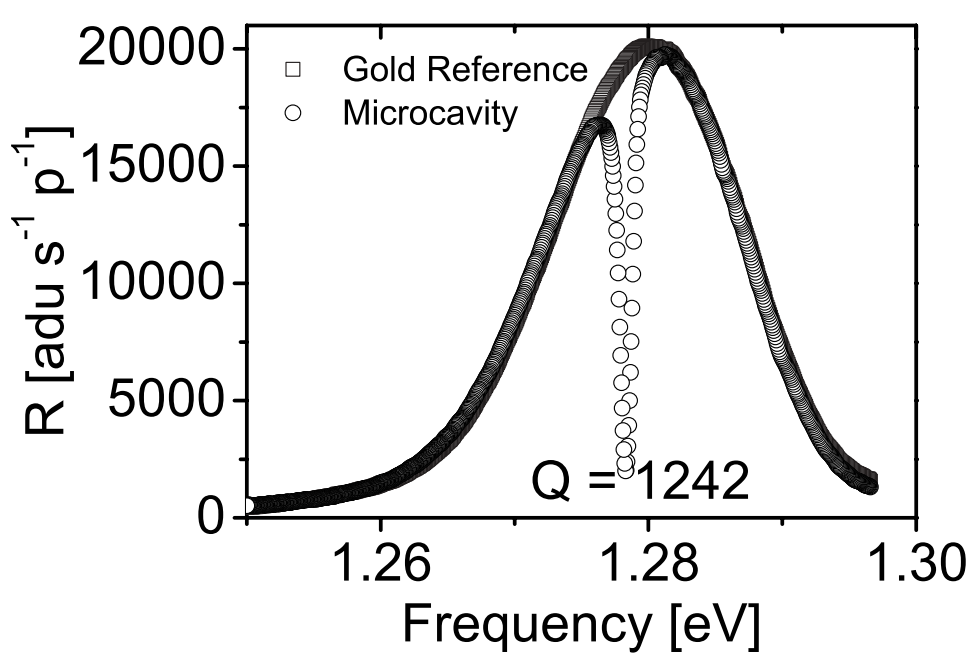

Figure 3.5: Reflected irradiance averaged over 500 probe pulses from a gold mirror $\square$ and from the microcavity $\bigcirc$. The $Q_{w, 0}^{\text {meas }}$ is 1242. The gold reference has been scaled by a factor $1 / 2$, and the fine Fabry-Pérot fringes have been removed by high-frequency filtering (see section 4.3).

the reflectivity at the far edges of the cavity resonance was earlier measured to be near $100 \%$ (see figure 3.1), we can correct for this misorientation as done in figure 3.5 without fearing a modified $Q_{w, 0}^{\text {meas }}$. In addition, the probe spectra varied in width by $1.3 \mathrm{meV}$ during our measurement, which we correlate with slowly varying laser power oscillations. The varying probe spectra caused the baseline of the reflectivity to fluctuate, and in addition to exhibit some varying slant. To reduce these effects, we averaged over 500 pulses. Finally, a thin window in front of the line detector gave rise to fine Fabry-Pérot fringes, which we removed by high-frequency filtering. We describe in detail how the data is processed in section 4.3.

\subsubsection{Dynamic linewidth}

Figure 3.6a shows reflectivity of the probe at different probe delays. To verify that heating effects and irreversible damage was not occurring at our pump intensities of $I_{\text {Pump }}=219 \pm 22 \mathrm{GWcm}^{-2}$ and pump frequencies $E_{\text {Pump }}=$ $0.73 \mathrm{eV}$, we averaged switched and unswitched spectra by halving the pump repetition rate, and operating the spectrometer in free-running mode for the duration of $1 \mathrm{~s}$. The resulting spectra are thus averages of switched and unswitched cavity resonances, with $1 \mathrm{~s} \cdot 0.25 \mathrm{kHz}=250$ spectra each. Because the switched and unswitched spectra are taken in turn, separated by a time interval of only $\Omega_{\text {rep }}^{-1}$, both spectra were taken under the same experimental conditions. The unswitched cavity resonance is clearly seen at $1.2783 \mathrm{eV}$. The width of the unswitched cavity resonance does not increase 

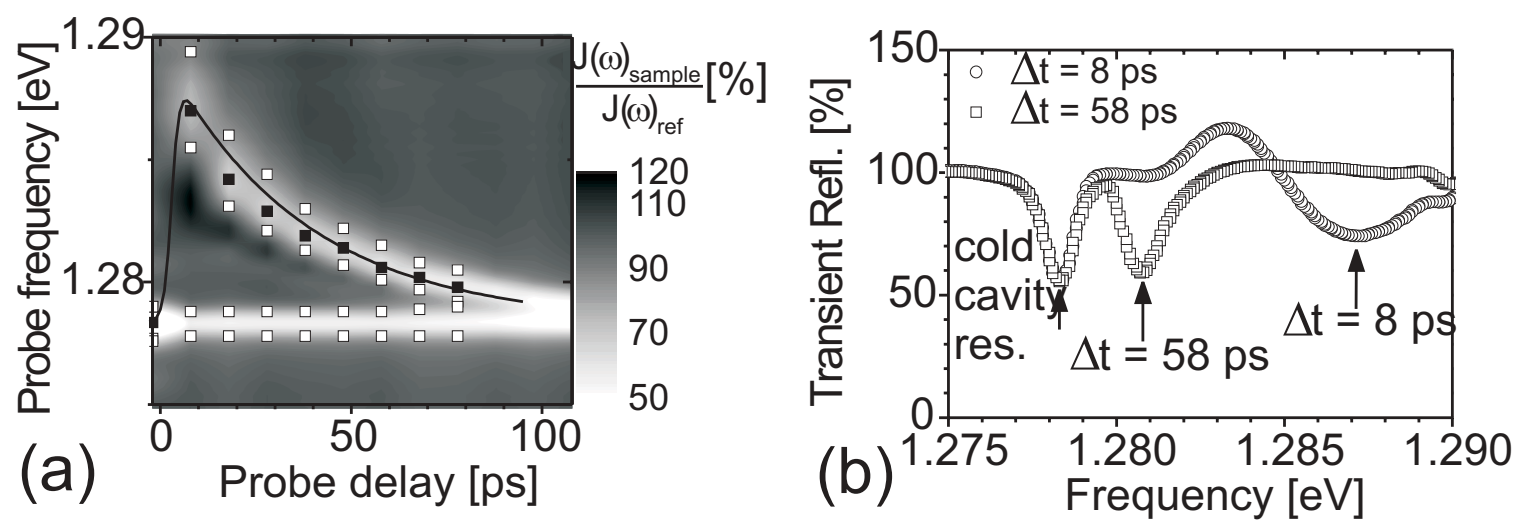

Figure 3.6.: (a) Transient reflectivity spectra $J(\omega) / J_{\text {ref }}(\omega)$ of averaged switched and cold cavity vs. probe delay, corrected for varying pump intensities and Fabry-Pérot fringes due to window in front of line detector. The switched cavity resonances ( $\mathbf{\square})$ are plotted with the half maxima of the red and blue edge ( $\bigcirc$ and $\square$ ) of the cold and switched cavities. While the pumped cavity shifts by 8.5 cold linewidths, there is no effect of heating or irreversible damage visible in the cold cavity. (b) Two cross-sections of (a), at $\Delta t=8 \mathrm{ps}$, and $\Delta t=58 \mathrm{ps}$, where the arrows indicate the resonances of the switched cavity. The pump and probe intensities are $I_{\mathrm{Pump}}=$ $219 \pm 22 \mathrm{GWcm}^{-2}$ and $I_{\text {Probe }}=7 \pm 2 \mathrm{GWcm}^{-2}$

in the slightest, as shown by the half maxima (open squares). We therefore conclude that there are no permanent damage or heating effects. The second trough emerging at $1.287 \mathrm{eV}$ at $\Delta t=6 \mathrm{ps}$ is the switched cavity resonance.

At positive probe delays the cavity shifts by as much as $8.7 \mathrm{meV}$, or 8.5 cold cavity linewidths, and decays with a $1 / e$ time constant of $\tau_{\text {off }}=38$ ps (black curve), although the agreement to an exponential is only moderate, and this decay time is around 20 ps shorter than determined previously. We will revisit the non-exponential and faster decay time shortly. To compare the magnitude of shift to figure 3.2 , we find that the ratio of frequency differences between the switched and cold cavity resonance is $8.5 \mathrm{meV} / 4.8 \mathrm{meV}=1.8$ times higher. The larger shift is mostly due to the higher pump irradiance by a factor $\frac{219 \pm 22 \mathrm{GWcm}^{-2}}{180 \pm 18 \mathrm{GWcm}^{-2}}=1.22 \pm 0.17$, leading to a $1.22^{2}=1.49 \pm 0.35$ larger shift. This is in good agreement to the measured factor 1.8. We thus conclude that the shift is compatible to excitation by two-photon absorption.

In figure $3.6 \mathrm{~b}$, we examine the reflectivity at two probe delays more closely. At the red edge of the switched cavity resonance we observe a reflectivity above that of the unpumped resonance. We will discuss this feature in Chapter 4. Moreover, the switched cavity resonance is not symmetric: its gradient at the red edge is substantially lower than at the red edge. 


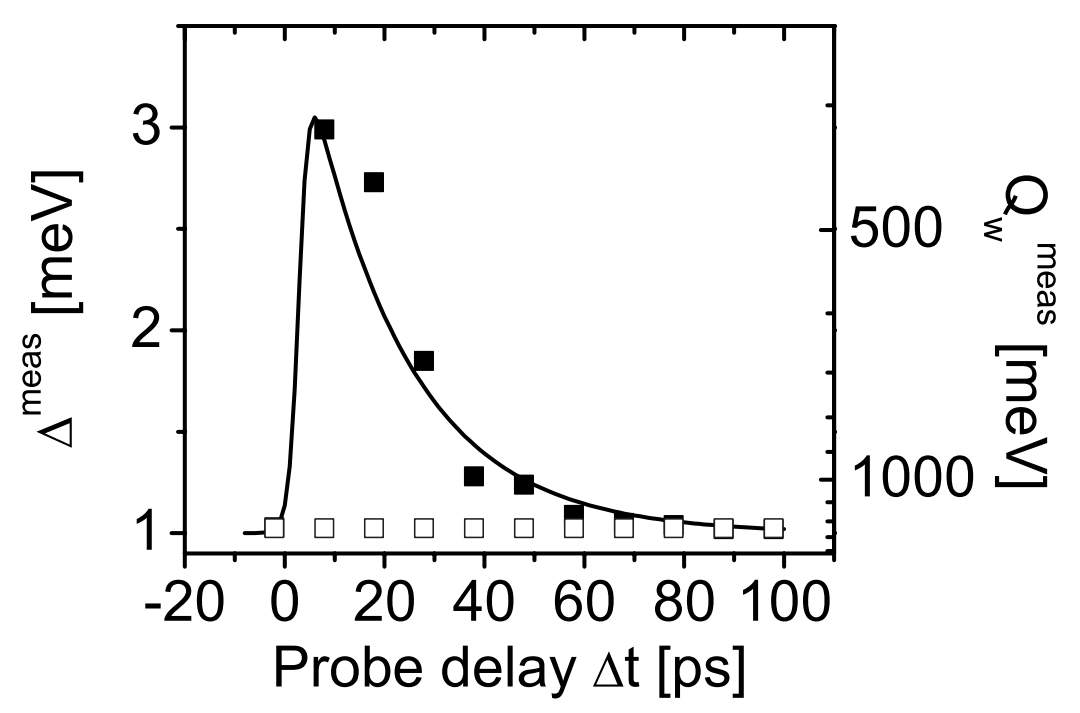

Figure 3.7.: Observed $\Delta^{\text {meas }}(\mathbf{\square})$ vs. probe delay from figure 3.6 with a guide to the eye (solid curve). On the right scale the corresponding $Q_{w}^{\text {meas }}$. The linewidth of the unpumped cavity resonance is shown by $\square$.

To quantify the broadening, the dynamic $\Delta^{\text {meas }}$ is plotted against probe delay (figure 3.7). At negative probe delays, $\Delta^{\text {meas }}$ is $1.03 \mathrm{meV}$ before increasing by a factor of 2.9 to $3 \mathrm{meV}$ at $\Delta t=6 \mathrm{ps}$. At $\Delta t=100 \mathrm{ps}$, the linewidth has decreased to its original value. We note that probing the dynamic cavity resonance at all $\Delta t$ at two distinct frequencies, or probing at all frequencies at a few $\Delta t$ as previously done in literature is insufficient to resolve these subtle dynamics. Moreover, broadening has hitherto been overlooked in literature. This neglect is surprising as the modulation depth, crucial to the performance of switching applications, depends critically on the amount of induced broadening.

Before interpreting the nonexponential decay and the assymmetric dynamic cavity resonance, we plot the relative linewidth versus the shift in cold linewidths (figure 3.8, open squares). For small shifts $\Delta E_{\text {cav }} / \Delta_{0}<2.5$, the broadening is $\Delta_{0}^{\text {meas }} / \Delta_{w}^{\text {meas }}<10 \%$. For larger shifts, the $\Delta^{\text {meas }}$ degrades rapidly, but has a point of inflection at $\sim 4 \Delta E_{\text {cav }} / \Delta_{0}^{\text {meas }}$ after which the decrease progresses less rapidly. We have calculated the expected relative decrease $Q_{w}^{\text {calc }} / Q_{w, 0}^{\text {calc }}$ with the same extended Drude model as previously (solid line), also showing the expected broadening using the carrier density from figure 3.4. The agreement is moderate, the calculated relation yielding 
larger broadening for $\Delta E_{\text {cav }} / \Delta_{0, w}^{\text {calc }}<3$, but lower broadening at larger shifts.

We can partially resolve the apparent disagreement by two additional calculations. First, because of the high recombination velocity $S_{1}$ at the AlAs on GaAs [121], but especially for the GaAs on AlAs interfaces $\left(S_{2}\right)$ [122], the carrier concentration gradient will be higher in the direction of the beam propagation than normal to it. Due to Fick's law, which relates the carrier gradient to the diffusion constant, the diffusion in the beam direction will be greatly enhanced compared to the directions normal to it. The carriers effectively move in one dimension. This is in contrast to refs. [70; 117] who studied bulk GaAs for their measurement. The diffusion is one dimensional and $1 / \tau_{D}$ is related to $N$, not $N^{1 / 3}$ (see section 3.3.3). In figure 3.8 we have plotted the relative decrease in $Q_{w, 0}^{\text {calc }}$ vs. relative linewidth by calculating $1 / \tau_{D}=\Gamma_{\mathrm{e}-\mathrm{ph}}+\gamma^{\prime} N$, where $\gamma^{\prime}=2 \cdot 10^{-12} \mathrm{~m}^{3} / \mathrm{s}$ (dashed curve). ${ }^{6}$ The agreement of the $1 \mathrm{D}$ diffusion model to our data is better. Both an onset of a plateau and the point of inflection are reproduced. The dominance of diffusion in beam direction is also proposed as the explanation why the decay time for probe frequencies close to the cavity resonance is so much longer. In the cavity, and at probe frequencies corresponding to the cavity resonance, the probe field is strongly enhanced, which it preferentially probes. For one-dimensional diffusion, the total decay rate can be written as [121]

$$
\begin{aligned}
1 / \tau_{\text {tot }} & =1 / \tau_{R}+1 / \tau_{N R}+\left(S_{1}+S_{2}\right) / d, \text { or } \\
\tau_{\text {tot }} & =\frac{d}{d \frac{\tau_{R}+\tau_{N R}}{\tau_{R} \tau_{N R}}+\left(S_{1}+S_{2}\right)}
\end{aligned}
$$

where $\tau_{R}$ and $\tau_{N R}$ are the radiative and non-radiative recombination rates in the bulk GaAs, respectively, and $d$ the thickness of the $\lambda$ cavity. Using an internal efficiency of $\geq 20 \%$ [121], and a radiative decay constant of $2 \cdot 10^{-16} \mathrm{~m}^{3} / \mathrm{s}$ [123], we find that the first term in the denominator is 2 to 3 orders of magnitude higher than the second for $1 \mathrm{~m} / \mathrm{s}<\left(S_{1}+S_{2}\right)<100$ $\mathrm{m} / \mathrm{s}$ [121]. While these values give a relative increase of only $1 \%$ for a four times thicker layer, the surface recombination velocities and bulk decay rates should be determined before conclusive evidence is presented. In conclusion, we have showed that the measured decay rate grows with layer thickness, in agreement to the data.

Second, the resonance is additionally broadened due to the finite shift accrued in the pulse interaction time. This can be seen in figure 3.6(a), where the minima of the dynamic cavity resonance are not centered between the

\footnotetext{
${ }^{6}$ In the absence of literature values, we normalize $\gamma^{\prime}$ to $\gamma$ at $10^{25} \mathrm{~m}^{-3}$.
} 
half maxima, but weighted to the unswitched resonance, the frequency the cavity resonance is relaxing to. For short probe delays, the interaction time is short, as the resonance has broadened due to free carrier absorption, but the cavity resonance velocity is large. At longer probe delays, the situation has reversed: the velocity has become negligible, but the interaction time is close to its unswitched value. The measured linewidth is thus a combination of the two effects. Calculations taking into account both the interaction time from the inverse linewidth derived above and the velocity from the derivative of the dynamic cavity resonance however were unable to reproduce the observed features. We thus conclude that while one dimensional electron diffusion is partially responsible for the plateau-like behavior in fig. 3.8, a simple model taking into account the frequency change of the resonance within the pulse interaction time does not further improve the agreement. Here, a more precise knowledge of the pulse interaction time might provide more insight.

The dependence of lineshape on pulse interaction time and resonance velocity is also the reason why the dynamic resonance lineshape is asymmetric and why the decay seems nonexponential: the lineshape seems to consists of several instantaneous troughs, each shifted by an infinitesimal amount in frequency, but weighted with the remaining irradiance in the cavity by an exponential. This new lineshape gives rise to a trough between $E_{\text {cav }}$ at the begin of the shift and $E_{\mathrm{cav}}+\delta$ when the exponential vanishes. The minima thus depend on the velocity, initial lineshape and interaction time, and cannot be identified with one given $\Delta t$. Therefore, the minima cannot be fitted with an exponential dependent on our $\Delta t$.

For future switching applications it is necessary that the broadening be limited, where the limitation is given by the required modulation contrast. In this context it would be useful to investigate experimentally what the broadening vs. shifting relation is for cavities of arbitrary $Q$. Initial calculations on TM models have indicated that high Q cavities are less susceptible to broadening for any given relative shift.

\subsection{Conclusions and recommendations}

In summary, we present unprecedented frequency-resolved dynamic behavior of a cavity resonance, with femtosecond resolution. Our experiments were optimized for spatially homogeneous switching by two-photon excitation. This feature facilitates a physical interpretation of the free carrier effects with an extended Drude model, including both the carrier density dependent Drude damping time and interband absorption. At pump and 
Figure 3.8: Measured relative decrease of quality factor vs. relative shift ( $\square)$, from fig. 3.6). For $\tau_{D}$ following the relation by Hügel et al. [70], the relation is given by the solid curve, and from fig. 3.4 by $\bigcirc$. For one dimensional diffusion $\left(\tau_{D} \propto N\right)$, we find a better agreement (dashed curve).

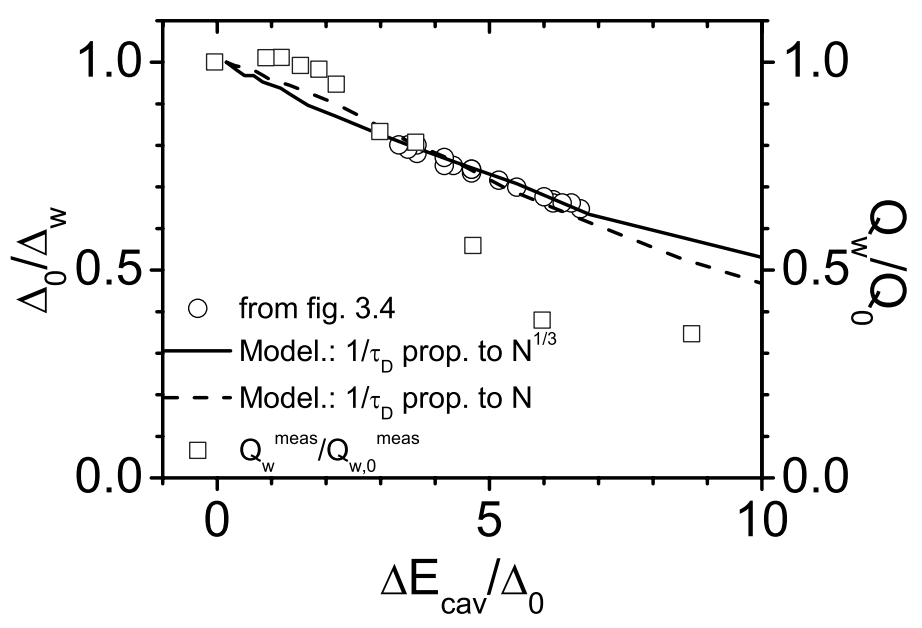

probe coincidence, we observe a strong decrease in reflectivity which we ascribe to non-degenerate two-photon absorption. In this case, we infer from a Transfer-Matrix model that $Q_{w}^{\text {calc }}$ decreases by a factor 12.9 . When the free carriers have thermalized $(\Delta t=6 \mathrm{ps})$, the greatest observed shift is as high as $4.8 \mathrm{meV}$, or 4.7 cold cavity linewidths.

By additionally spectrally resolving the reflected probe pulses, both the shifted cavity resonance as well as its lineshape can be determined. At shifts of up to $8.7 \mathrm{meV}$, or 8.5 cavity linewidths, the resonance broadens by up to a factor 2.9, the width being a combination of absorption due to free carrier absorption and the finite probe pulse duration coupled with the velocity of the dynamic resonance. We show that this broadening remains $<10 \%$ for shifts of up to 2 linewidths. While literature often focusses on on/off switching obtained by measuring transmittance of a narrowband light source at the unpumped cavity resonance, we show for the first time that broadening due to free carrier absorption may intrinsically limit performance of this switching scheme if shifts in excess of 2 linewidths are desired. A Transfer-Matrix model coupled with the extended Drude model is used to estimate the cavity resonance broadening vs. shift. When movement of carriers is one dimensional, as is shown to be the case, the model yields good agreement, which can further be improved by closer inspection and optimization of parameters. Additional preliminary calculations indicate that cavities of greater quality are less susceptible to broadening for any given shift in cold linewidths.

For further analysis it seems essential to understand the relation between the dynamic width of the resonance and its dwell time. Not only might 
knowledge of this relationship elucidate apparent discrepancies between theory and measurement, but it would enable the tailoring of cavities to match the needs of certain time-resolved experiments. Determination of $Q$ is especially significant for strongly switched cavities, in which the linewidth is not only affected by free carrier absorption, but also by the finite recombination velocity in addition to the finite probe duration. Determination of the intrinsic linewidth is complicated by finite NA, surface roughness, spatial distribution and finite absorption in the GaAs even well below the bandgap. 



\section{Chapter 4}

\section{Dynamics of light in an ultrafast switched cavity}

\subsection{Introduction}

Cavities are photonic structures which can strongly confine light. Photonic crystals hosting cavities are of special interest, as not only can high quality factors $(Q)$ be achieved in these cavities [124], but also small mode volumes $V$, which is advantageous for high Purcell factors [99]. The $Q$ factor, describing the measure of a mode's time duration to store energy, originates from mechanical oscillators. Cavities have been hailed as means to control emission of light [91], to emit single photons [125], to enhance light-matter interaction [92], and to trap and release photons [83; 86]. For cavities to be useful in applications such as optical modulators $[78 ; 80 ; 113]$ or wavelength converters [82], it is necessary to switch them on ultrafast time scales. While switching of cavities has often been demonstrated $[81 ; 100 ; 126]$, the dynamics of light during the switching process has scarcely been described analytically. This scarceness of theory is surprising, as switching cavities has been viewed in analogy with a simple system such as a resonant guitar string, which is made to change its resonance by dynamically changing the length of the string [84; 103]. Bret et al. not only succeeded in changing the bandwidth of a captured pulse in a macroscopic cavity, but described the process theoretically [86]. A similar experiment was later performed on a micrometer scale [87]. Yacomotti et al. mathematically described spectral features observed in reflection when a pump pulse switches a cavity containing a probe pulse [88]. While finite difference time domain (FDTD) calculations have produced results that match experiments well [103] or predict results later to be verified experimentally [84; 85], they do not provide physical insight on the underlying phenomena. In this chapter, we will show 
pump-probe experiments performed on a planar microcavity at both negative and positive probe delays, and present a physical model which explains many of the observed results.

\subsection{Experimental setup and linear reflectivity}

The sample and experimental setup have been described in detail in the previous chapter, in which the detection is frequency resolved. Figure 3.5 shows the spectrum of a probe pulse reflected from a gold mirror. The spectral width of $17.2 \mathrm{meV}$, or $1.35 \%$ corresponds excellently to the relative width of $1.33 \%$ measured on a different occasion with an alternative spectrometer. For a Fourier-limited Gaussian pulse, the pulse duration should be $\tau_{P}=2 \pi 0.441 /\left(0.0133 \omega_{L}\right)=106 \mathrm{fs}$, where $\omega_{L}$ is the carrier frequency of the laser of $\omega_{L}=1.95 \cdot 10^{15}$ in $\mathrm{rad} / \mathrm{s}$. Comparing the Fourier-limited pulse duration to the measured duration of $140 \pm 10 \mathrm{fs}$, we conclude that the pulse has some chirp.

In the same figure, we also show the spectrum of a probe pulse reflected by the microcavity. The deep trough at $E_{0}=1.2783 \mathrm{eV}$ is due to the cavity resonance, which has a FWHM width of $\Delta_{0}^{\text {meas }}=1.03 \mathrm{meV}$, or a quality factor estimated from the spectral width of $E_{0} / \Delta_{0}^{\text {meas }}=Q_{w, 0}^{\text {meas }}=1242$. From this $Q$ value, the $1 / e$ intensity cavity time (or photon dwell time $\tau_{\text {cav }}$ ) is given by $Q / \omega_{0}=640 \mathrm{fs}$, where $\omega_{0}$ is the cavity resonance in units of $\mathrm{rad} / \mathrm{s}$.

\subsection{Data processing}

In most of our pump-probe switching experiments, we measure the reflected pulse's spectrum for both positive as well as negative probe delays, averaged over switched and unswitched spectra. For details see section 3.4.2. The averaging serves to verify that no degradation was occurring, as would immediately be visible by the changed unswitched cavity lineshape. Figure 4.1 shows such an averaged spectrum divided by a reference. We observe fast oscillations of a period $0.33 \mathrm{meV}$, which we attribute to Fabry-Pérot fringes originating from the thin window in front of the line detector. The oscillations are removed by smoothing with an FFT filter removing all frequency components greater than $1 /(0.36 \mathrm{meV})$. We verified that the modulation depth of the cavity resonance was not affected. Moreover, at frequencies outside of the cavity resonance, the transient reflectivity is close to $50 \%$, and exhibits a slight slant, which changed in time. The sub-unity reflectivity 


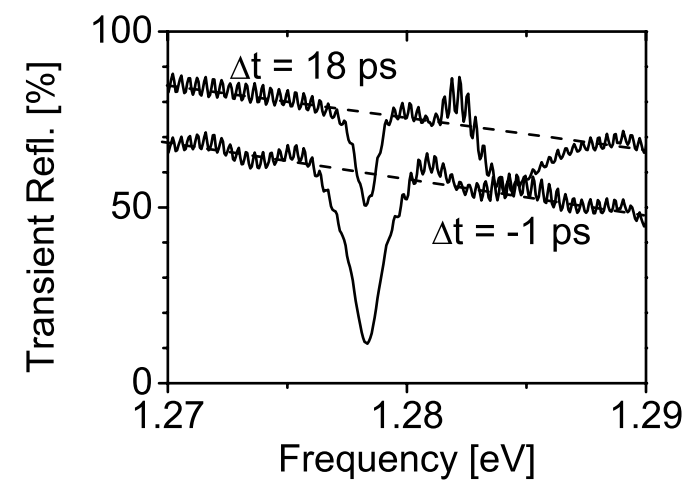

Figure 4.1.: Raw spectra of an averaged switched and unswitched cavity resonance at probe delays of $\Delta t=-1 \mathrm{ps}$ and $\Delta t=18 \mathrm{ps}$, divided by a reference. The slant is due to the laser frequency and irradiance varying in time, while the sub-unit offset is caused by a slight misalignment. The slant is numerically corrected for by dividing through a straight line (dashed line), the fine oscillations of period $0.33 \mathrm{meV}$ were removed by high-frequency filtering.

is attributed to a slightly misaligned reference mirror. The changing slant is due to long-term laser carrier frequency oscillations of $1 \mathrm{meV}$, which were coupled to irradiance fluctuations. The change in carrier frequency can be observed in figure 3.5: upon close inspection of the reference spectra's red and blue tails, a minute blue shift with respect to the cavity spectrum can be observed. The slant is corrected for by ratioing the measured spectrum to a straight line that is drawn through the spectra outside the cavity resonance (see figure 4.1). We had previously measured linear reflectivity with a broad-band Fourier-Transform Interferometer, and had confirmed on numerous occasions that the sample's reflectivity was close to $100 \%$ outside the cavity resonance (see, e.g., fig. 1 in the previous chapter).

\subsection{Negative probe delay}

Figure 4.2(a) shows the differential reflectivity as a function of probe delay and frequency. Around $E_{\text {rmcav }}=1.279 \mathrm{eV}$, which is the cavity resonance for this measurement, we observe a large positive differential reflectivity at negative probe delays, while at either side of the resonance slightly negative $\Delta R / R$ can be observed. These spectral features can only be explained by a broadening of the switched cavity resonance before the incidence of the 
probe. We speculate that the reason for this broadening lies in the probe pulse reflecting from the sample's rear side, and encountering the altered cavity lineshape, which is the result of the pump having arrived in the mean time. Similar behavior has also been encountered in the previous chapter. As from $\Delta t=-0.5 \mathrm{ps}$, the additional large negative $\Delta R / R$ at the blue edge of the cavity resonance indicates that the carriers created by the leading edge of the pump pulses are probed by the trailing edge of the probe pulses, which results in a shift of $0.7 \mathrm{meV}$, or around 0.7 cold cavity linewidths. At coincidence, the cavity resonance is estimated to shift in excess of 2 cold cavity linewidths. Most markedly however, are the presence of fringes which occur on either side of the cavity resonance. With decreasing negative probe delay, they fan out until they diverge and disappear at coincidence. To investigate the fringes further, we have plotted the transient reflectivity in figure 4.2(b), averaged over switched and unswitched spectra. The unswitched cavity resonance in this measurement is $E_{\text {cav }}=1.2783 \mathrm{eV}$, and otherwise experimental conditions are equal to previously. For $\Delta t=-2.1 \mathrm{ps}$, the unswitched cavity resonance can be observed at $E_{0}=1.2783 \mathrm{eV}$. At either side, fringes are observed with a spacing of $2.0 \mathrm{meV}$. With increasing $\Delta t$, these oscillations become more widely spaced. The fanning out of the fringes in fig. 4.2(a) thus corresponds to an increasing period of the fringe spacing. At $\Delta t=-0.1 \mathrm{ps}$, the onset of the switched cavity resonance can clearly be observed at 1.2818 $\mathrm{eV}$. At this stage, the modulation depth of the unswitched cavity has decreased by a factor 1.4. For large positive probe delays, the modulation depth decreases by a factor 2 as it is sharing spectrometer counts with the switched cavity.

The change in fringe spacing with probe delay is plotted in figure 4.3. While the fringe spacing increases only slightly from $2.4 \mathrm{meV}$ from $\Delta t=-3$ ps to $-2 \mathrm{ps}$, the fringe spacing increasing rapidly for smaller negative probe delays, and finally seems to diverge at coincidence. A naive interpretation of the fringes is that Fabry-Pérot fringes form. At large negative probe delays, the spacing of $2.4 \mathrm{meV}$ corresponds to an optical thickness of around 150 $\mu \mathrm{m}$, just less than the substrate thickness. At $\Delta t=-0.5 \mathrm{ps}$, the apparent thickness would have had to have decreased to only $25 \mu \mathrm{m}$ if the Fabry-Pérot interferences were truly the cause of the observed fringes. Since such a large physical change is absurd, we conclude that the formation of the fringes is not due to Fabry-Pérot fringes of any possible layers. 

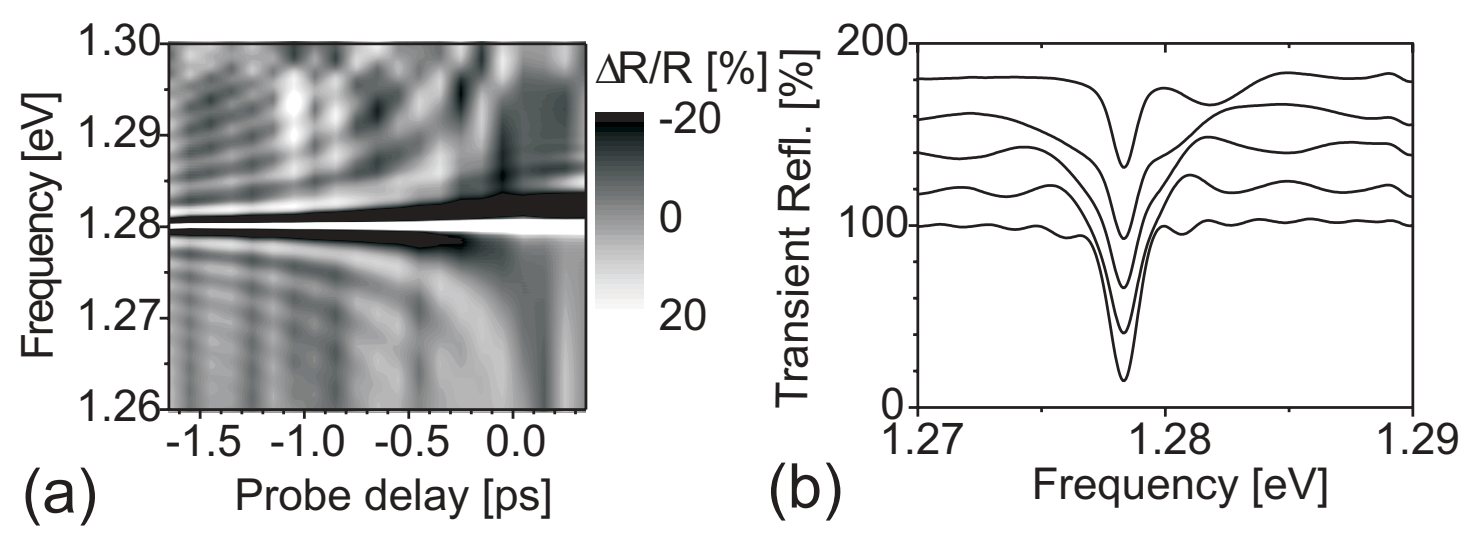

Figure 4.2.: (a) Differential reflectivity at negative probe delays. The signal is not averaged over pumped and unpumped spectra. The dynamic range of the plot has been limited to enhance the visibility of the fringes. (b) Averaged switched and unswitched spectra divided by a reference. The spectra have successively been offset by $20 \%$ points from bottom to top with decreasing negative probe delay: $\Delta t=-2.1 \mathrm{ps},-1.1 \mathrm{ps},-0.8 \mathrm{ps},-0.5 \mathrm{ps},-0.1 \mathrm{ps}$. The pump and probe intensities are $I_{\text {pump }}=219 \pm 22 \mathrm{GWcm}^{-2}$ and $I_{\text {probe }}=7 \pm 2 \mathrm{GWcm}^{-2}$.

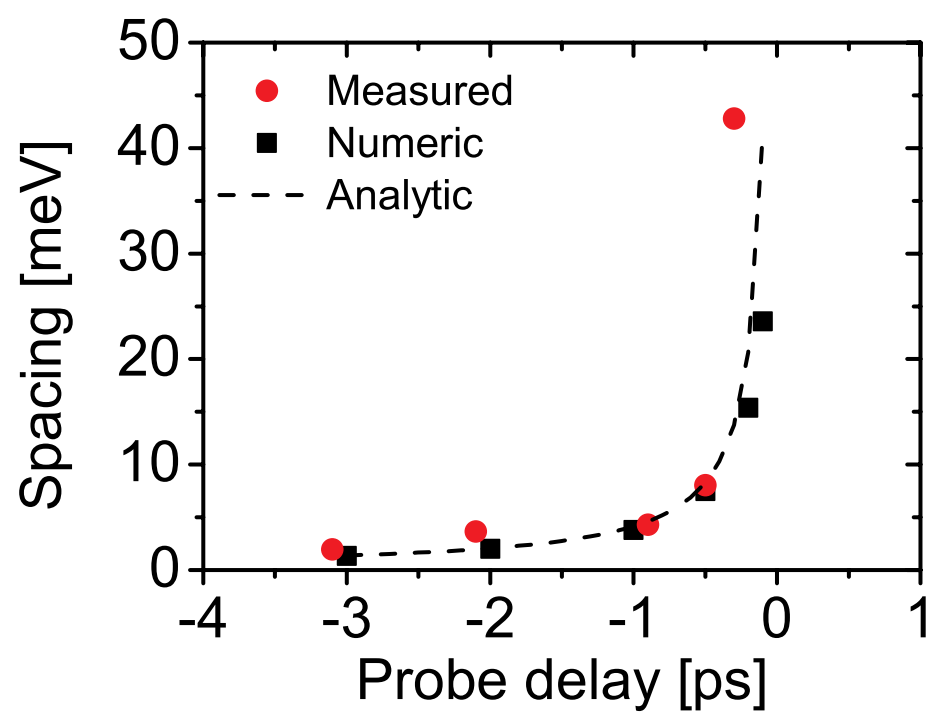

Figure 4.3.: Fringe spacing versus probe delay. 


\subsubsection{The intuitive model}

Before giving a mathematical description of the spectra, we will give an intuitive explanation of the physics behind the observed effect [127], see figure 4.4(a) - (d). A probe pulse is incident on an empty cavity at time $t_{1}(4.4(\mathrm{a}))$. At time $t_{2}$, most of the probe gets reflected by the microcavity (b). In the frequency domain, this large reflection is caused by the large probe bandwidth, which greatly exceeds that of the resonance. Still, light resonant with the cavity resonance will recirculate inside the cavity. At a time $t_{3}$, the pump is incident. At this time the refractive index of the GaAs starts to decrease because of the thermalizing free carriers, thereby reducing the contrast in the Bragg stacks. Not only does the change in index give rise to a shifting resonance, but the surrounding Bragg stacks become less reflecting, and thus more transmitting (c). Therefore, the field in the switched cavity will leak out fast, on timescales which are much shorter than $\tau_{\text {cav }}(\mathrm{d})$. This quickly leaking field can be considered as a pulse. We thus have two consecutive pulses of light separated in time by $\Delta t$. The first directly reflected pulse has a duration $\tau_{P}$, while the shape of the second pulse is a function of the time-dependent dwell-time at the frequency of the probe field due to the shifting cavity resonance. The Fourier Transform of two pulses separated by $\Delta t$ yields two oscillating frequency components, which beat with a period $1 / \Delta t$, similar to what we observe. The amplitude of this beating depends on the shape and duration of the two pulses.

\subsection{Positive probe delay}

Figure 4.5(a) shows the transient reflectivity vs. probe delay, where the spectra have been averaged over switched and unswitched. The unswitched resonance sits at $E_{0}=1.2783 \mathrm{eV}$. At coincidence, the switched cavity resonance $E_{\text {cav }}(t)$ starts demerging from the unswitched resonance and blue shifts in a time $0<\Delta t<\tau_{\text {on }}=3$ ps to $E_{1}$ by $11.7 \mathrm{meV}$, or 11 cold linewidths. During this time the resonance broadens to $6.3 \mathrm{meV}$, or by a factor 6 with respect to the unswitched cavity. Most notably however, the magnitude of the transient reflectivity in the spectral range 1.28 to $1.285 \mathrm{eV}$ is clearly in excess of unity. In figure 4.5(b), we have plotted several spectra at positive probe delays. At $\Delta t=0.9 \mathrm{ps}$, the resonance is very broad, but shows no sign of enhanced reflectivity. As soon as the cavity resonance has ceased blue shifting, however, we observe a transient reflectivity of $115 \%$ for $\Delta t=7.9 \mathrm{ps}$. This value translates to $130 \%$ if we correct for the averaging. 


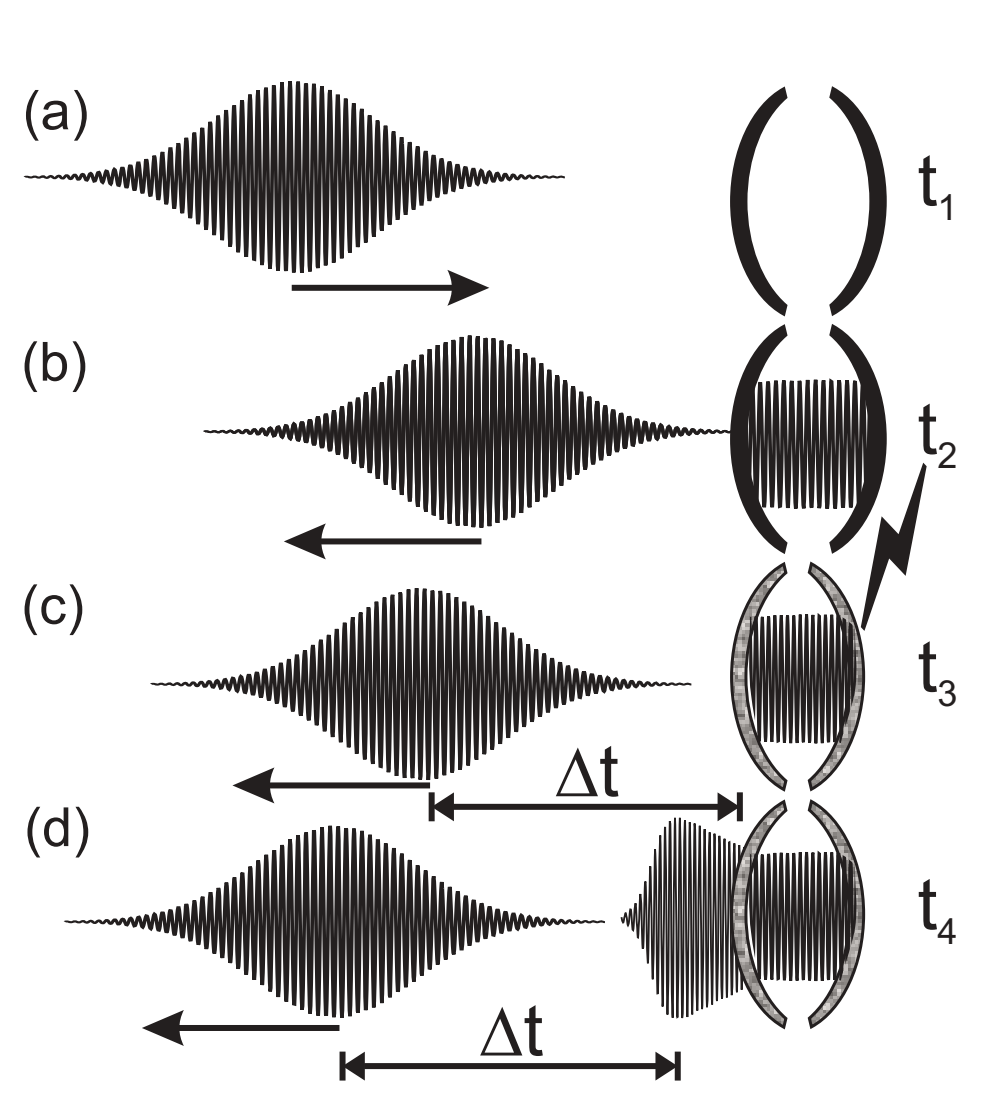

Figure 4.4: Cartoon illustrating the origin of fringes at negative probe delays. In (a), a probe pulse is incident on the cavity at $t_{1}$. (b) Because of the probe's much larger bandwidth compared to that of the cavity, most of the probe gets reflected at $t_{2}$. (c) A short pump pulse changes the resonance conditions at time $t_{3}$, and field in the cavity starts leaking. (d) At time $t_{4}$, there are now two consecutive pulses: the directly reflected pulse and the released pulse from the cavity, separated by $\Delta t$, the pump-probe delay. Together, the pulses yield fringes in a Fourier Transform.

With increasing probe delay, the magnitude of the enhancement decreases, and cannot be observed for $\Delta t>40 \mathrm{ps}$. The raw spectra, i.e., not corrected for our experimental conditions, display clear peaks blue of the unswitched cavity resonance, see figure 4.1. We therefore conclude that the excess reflectivity is no artifact of the data processing. The transient reflectivity in excess of $100 \%$ does not necessarily contradict energy conservation, as the spectral density of the field in the shifting cavity might change due to the transient refractive index. For $\Delta t>\tau_{\text {on }}$, the resonance decays with an exponential time constant $\tau_{\text {off }}=57 \pm 2$ ps to its unswitched value due to carrier recombination (see fig. 3.6(a)). The unswitched cavity resonance retains its original width of $\Delta_{0}=1.03 \mathrm{meV}$, confirming that no degradation is taking place.

\subsection{Physical mass and string model}

Our goal is to understand quantitatively the observed phenomena, that is, the fringes at negative probe delays, and the reflectivity in excess of unity of positive probe delays. To this end, we model the cavity and the Bragg stacks as a mass $m$ suspended by a spring with a constant $k(t)$, the mass 

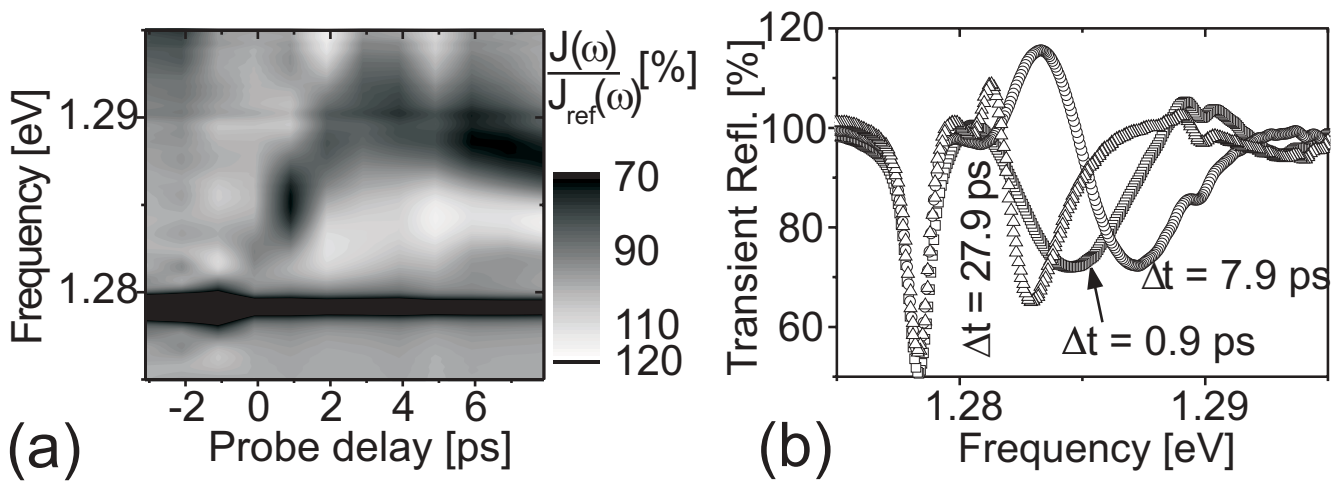

Figure 4.5.: (a) Transient reflectivity at positive probe delays, averaged over switched and unswitched spectra. (b) Transient reflectivity spectra at three different probe delays.

being sandwiched by two attenuators, see figure 4.6. The latter attenuate the elongation of two attached strings of tension force $\sigma$ and unit mass $\rho$ to the mass and back again by a factor $f(t)$. A transverse wave of elongation in the y-direction $A_{i}(x, t)$ travelling in the $x$-direction is incident from the left and drives the mass which as consequence has an elongation $y(t)$. The wave in the model is identified with the incident probe beam, the mass suspended by the spring with the cavity, and the attenuators are identified with the surrounding Bragg stacks. At the position of the mass, $x=0$, and we define $A_{i}(x=0, t)=A_{i}(0, t)=A_{i}(t)$. Only a fraction $f(t)$ of the driving force $F(t)$ exerted by the incoming wave is passed on to the mass and vice versa. This force $F_{d}(t)$ is equal to

$$
F_{d}(t)=2 f(t) \sigma(\partial / \partial x) A_{i}(t)=2 f(t) \sigma / c(\partial / \partial t) A_{i}(t)
$$

where the wave's speed is $c=\sqrt{\sigma / \rho}$. Damping of the mass occurs via the force exerted on the string. This force is

$$
F_{\gamma}(t)=2 f^{2}(t) \sigma / c(\partial / \partial t) y(t)
$$

where one factor $f(t) c^{-1}(\partial / \partial t) y(t)$ is the x-derivative of the amplitude, the other factor $f(t) \sigma$ is the force exerted on the string. Damping due to the mirrors is thus $2 \Gamma_{r}(t)=2 f^{2}(t) \sigma / c$.

Intrinsic damping in our experiment due to, e.g., out of plane scattering or absorption has not been taken into account for two reasons: first, we showed in Chapter 3 that $Q_{w}$ is close to $Q_{w}$ limited by wavevector spreading for the NA of our system, and therefore intrinsic scattering in the unswitched case 


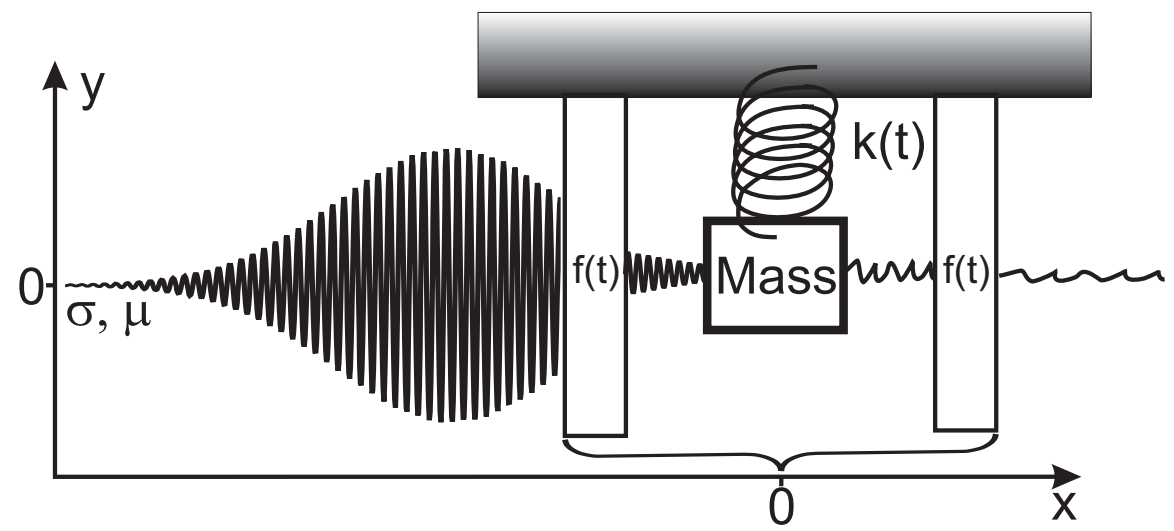

Figure 4.6.: The mass and spring model employed to calculate the reflected amplitude of a cavity changing its resonance in time.

is below the experimental error. Second, in the switched case, the transmission through the Bragg layers is increased by decreasing the refractive index contrast. Since the transmission goes with the exponent of the dielectric difference, even very small changes in the difference can change $f(t)$ by several orders of magnitude. In order to sustain the hypothesis that the resonance width is largely determined by the Bragg stacks' index contrast, and not by the free carrier absorption in the cavity, we performed Transfer Matrix calculations. These calculations show that the linewidth due to switching the Bragg stacks only is very close to the linewidth obtained for switching both the cavity and the stacks. Therefore, free carrier absorption in the cavity can be neglected.

The equation of motion can then be written as follows,

$$
\left(\partial^{2} / \partial t^{2}\right) Y(t)+2 \Gamma_{r}(t)(\partial / \partial t) Y(t)+\omega_{\text {cav }}^{2}(t) Y(t)=\sqrt{\Gamma_{r}(t)}(\partial / \partial t) A_{i}(t),
$$

where we have set $\Gamma_{r}=f(t)^{2} \sigma / c$, and $y(t)=Y(t) \sqrt{\sigma / c}$. At $x=0$, the sum of the incident and the scaled reflected wave $f(t) A_{r}(t)$ is equal to $y(t)$, or $A_{r}(t)=\sqrt{\Gamma_{r}} Y(t)-A_{i}(t)$. In comparison to our experiments, we can identify the optical irradiance $J(\omega)$ in equation 6 in Chapter 2 with $J(\omega)=$ $\left|\int_{-\infty}^{\infty} A_{r}(t) \exp (i \omega t) d t\right|^{2}$.

\subsection{Results: mass and spring}




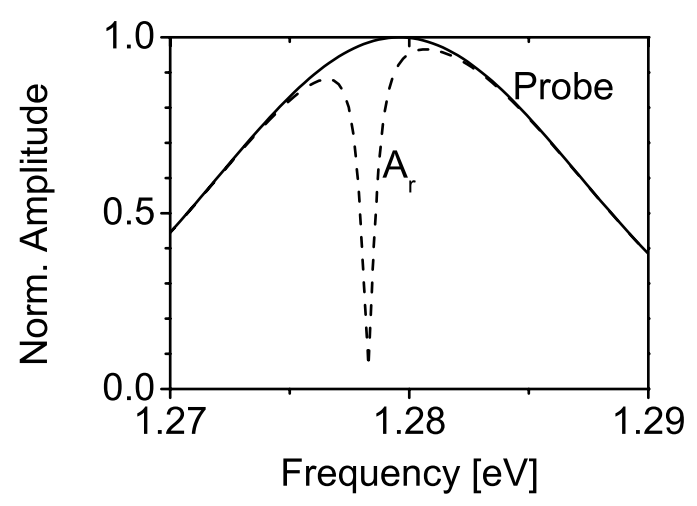

Figure 4.7.: Static amplitude reflection coefficient $A_{r}$ and probe spectrum calculated from the mass and spring model.

\subsubsection{Static case}

We solved equation 4.3 numerically by driving the mass with the derivative of a non-chirped Gaussian pulse with carrier of frequency $\omega_{L}$ and a FWHM $\tau_{G}=\sqrt{2} \tau_{P}$. Both the resonance and the linewidth are set to the unswitched cavity resonance and width, respectively. Care must be exercised with $\Gamma_{r}(t)$ : equation 4.3 can be written as $\ddot{x}+a \gamma \dot{x}+\omega_{0}^{2} x=0$, which has an intensity FWHM of $a \gamma$. We thus identify $2 \Gamma_{r}(t)$ with $\Delta^{\text {meas }}$. Figure 4.7 shows the probe and amplitude reflection spectra. Both spectra have been normalized to the maximum value of the probe. At the resonance $A_{r}$ shows a pronounced trough which extends to 0 , limited by the frequency resolution of the data. The width of this trough is $0.7 \mathrm{meV}$, which translates to a calculated width of $\sqrt{2} \cdot 0.7 \mathrm{meV}=1 \mathrm{meV}$, in excellent agreement to $\Delta_{0}^{\text {meas }}$. From the good agreement of the model to the static measurement we conclude that the model serves as a good basis for further calculations.

\subsubsection{Dynamic case}

For a few salient time delays, we solve equation 4.3. As dynamic resonance and linewidth we strictly use measured parameters as extracted from the lineshapes at positive probe delays (see fig. 4.5). We emphasize that we have no free parameters. Figure 4.8(b) shows the calculated transient reflectivity. At $\Delta t=-2 \mathrm{ps}$, we observe fringes at the blue edge of the unshifted cavity with a spacing of $2.1 \pm 0.2 \mathrm{meV}$, corresponding to a time delay of $1.96 \pm 0.2$ ps. The fringe spacings are plotted for all calculated time delays in figure 


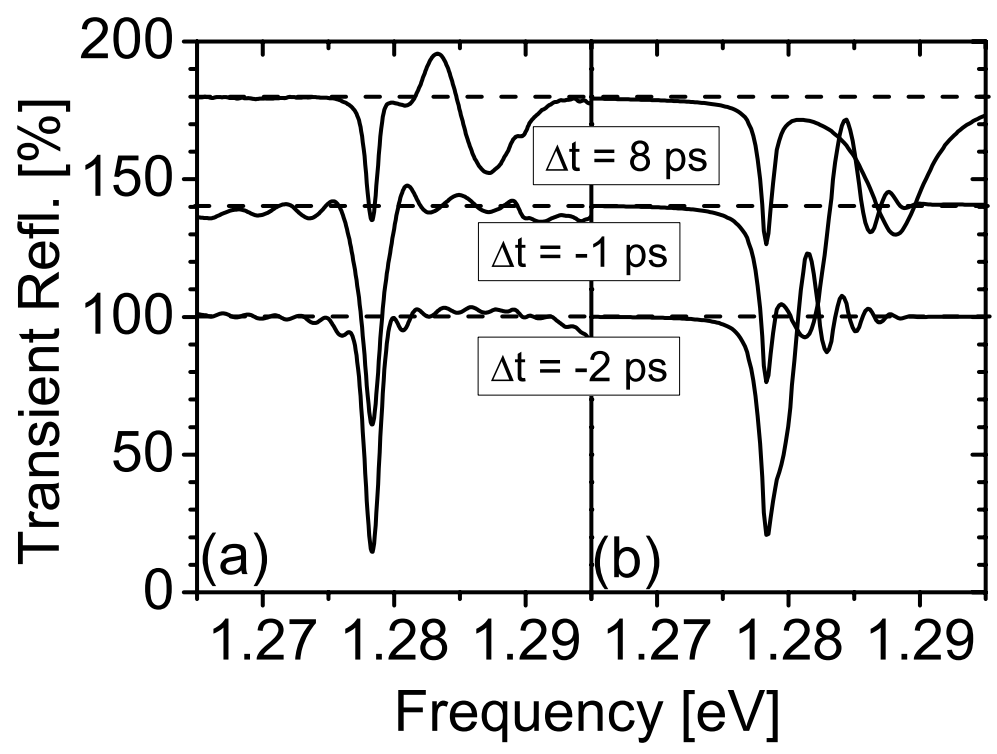

Figure 4.8.: (a) Measured transient reflectivity as in figs. 4.5 and 4.2. (b) Calculated spectra. All spectra have been offset by $40 \%$ for enhanced clarity.

4.3 , and are found to agree excellently both with the inverse probe delay and the measurements. The magnitude of the fringes clearly exceeds unity, a feature that is supported by our measurements. At $\Delta t=-1 \mathrm{ps}$, the maximum fringe height has decreased. The shifted trough of the switched cavity can already clearly be identified at $1.28 \mathrm{eV}$. For a positive probe delay of $\Delta t=10 \mathrm{ps}$, the unswitched and switched troughs have now separated, and the whole spectrum is below unity reflectivity. In contrast, figure 4.8(a) shows the measurements as shown in figs. $4.5(\mathrm{~b})$ and $4.2(\mathrm{~b})$. While there is similarity in the fringe spacings and the resonance positions, the exact shape of the spectra show marked differences. For example, no enhancement can be observed at positive probe delays, and the fringes cannot be observed at the red side of the unswitched cavity resonance. The following section will give some possible reasons why the model is unable to produce some of the observed features.

\subsection{Delimitations}

In this section we will discuss some limitations of the mass and spring model, and analyze what physical phenomena could lead to the discrepancy of the 
model with respect to the measurements. While the period of the fringes at negative probe delays is equal to the inverse of the delay of the pump pulse to the probe pulse, their amplitude properties depend on the two pulses' frequency distribution. As an example, two Gaussian pulses of field FWHM duration $\tau_{G}$ centered around frequencies $\omega_{1}$ and $\omega_{2}$ incident at time $t_{1}$ and $t_{2}$ respectively, give rise to beats in frequency space with a period of $1 /\left(t_{2}-t_{1}\right)$, but additionally damped by a Gaussian centered at $\omega_{\mathrm{av}}=\left(\omega_{1}+\omega_{2}\right) / 2$ and FWHM width of $\left(8 \ln (2) / \tau_{P}\right)$. It is thus clear that minimum damping should occur at $\omega_{\mathrm{av}}$, or that the fringe amplitude at the blue edge of the unswitched cavity is higher than at the red side. For chirped pulses as in this experiment, however, this relation is not generally valid, and the fringe amplitude may take any form.

A second unknown are the instantaneous values of $E_{\text {cav }}(t)$ and $\Gamma_{r}(t)$. This issue has been discussed in section 3.4.2. The measured cavity resonance and width will be a function of the resonance velocity $d E_{\text {cav }}(t) / d t$. A comparison between the $E_{\text {cav }}(t)$ and $\Gamma_{r}(t)$ inserted into the model and the dynamic resonance and width from the model showed that the latter had a noticeably smaller gradient. This observation sustains our hypothesis that the instantaneous and measured resonance and width deviate from one another. In future, this deviation could be removed by repeatedly solving equation 4.3, iterating the given $E_{\mathrm{cav}}(t)$ and $\Gamma_{r}(t)$ so that the resulting resonance and widths match the measurements.

A third factor is the possibly different carrier recombination times in the $\lambda$ cavity and in the Bragg stacks, which might lead to a carrier inhomogeneity (see Chapter 3, section 3.4.2), for which interesting spectral features have been predicted [84] and measured [85]. This inhomogeneity is not amenable to inclusion into our simple mass and spring model, but could be modeled in a finite difference time domain calculation.

We conclude that the mass and spring model gives insight into the physical mechanisms of cavity switching, reproducing most measured results. Experimental unknowns such as chirp, exact resonances and widths, and inhomogeneity prevent us from fully understanding the underlying switching mechanisms. More information on the precise experimental configuration is necessary before a further analysis can be done.

\subsection{Conclusions and recommendations}

Spectrally resolved measurements have been performed on switched cavities, for both negative as well as positive time delays. For the first time we 
present an intuitive model of photonic switching, which exploits the analogy with a mechanical oscillator, and quantitatively describes the measured spectra while giving physical insight into the switching process. At negative probe delays spectral fringes were observed which are caused by two pulses interfering with one another, the first one being the pulse reflected from the cavity, the second being a trapped pulse released from the cavity on arrival of the pump pulse. The fringe spacing is shown to scale as $1 / \Delta t$, and agrees excellently to our proposed model. At positive probe delays, the cavity resonance shifts by 11 linewidths within 3 ps, and broadens by a factor 6 in the same time which is well described by our model. At the red edge of the switched cavity, we observe transient reflectivities of up to $31 \%$. While the enhanced reflectivity cannot be understood from our model, we note that several unknown parameters are yet to be analyzed. 



\section{Femtosecond versus picosecond switching of Si woodpile photonic crystals}

\subsection{Introduction}

The interest to optically switch photonic structures has recently been gathering momentum due to the inherent rapidity of the process. While the switching speed of conventional transistors is ultimately limited by heat dissipation, no such limitation is known for optical systems in the absence of absorption. Often, the dispersion of photo-induced free carriers is exploited to induce ultrafast changes in the photonic structure's optical properties. Then, propagation switching, optical modulation, trapping and releasing photons, frequency- and bandwidth conversion, and even ultrafast switching of the density of states is possible [81-83; 86; 103].

Free carrier switching has many advantages to other sorts of switching. First, the carriers are generated within ps, allowing for potentially high repetition rates [128]. Indeed, currently many efforts are devoted to decreasing the timescales of switching in polycrystalline silicon, achievable through implanting additional recombination centers such as ions $[100 ; 113 ; 129]$. In contrast, molecular reorientation in liquid crystals responsible for changes in refractive indices gives rise to timescales of three to six orders of magnitude slower [130-132], rendering them useless in ultrafast devices. Thermal and mechanical tuning suffer the same fate $[84 ; 102]$. Second, generation is possible all-optically, and is so amenable to be performed by on-chip diode lasers. Third, the induced refractive index change is large compared other techniques. To compare, the only other switching technique of comparable speed is the optical Kerr effect, which has non-linear coefficients orders magnitude lower [133-135]. Finally, the change can scale linearly or quadrat- 
ically, depending on the relative frequency of the pump pulse to the switched semiconductor. Therefore, large dynamic ranges can be achieved with only small irradiance changes.

Recently, several authors [136-138] have claimed to have experimental evidence of the Kerr non-linearity. The reasoning in all cases is the instantaneous nature of the effect, with a decrease in reflectivity or transmission coinciding with the cross-correlation of the pump and probe pulses [137]. The duration of the pump and probe pulses is then the limitation to potential repetition rates, instead of the recombination times of the free carriers. Kerr switching could increase the repetition rate from $\mathrm{GHz}$ to THz. Large measured values of $n_{2}$, the nonlinear refractive index, could spark new interest in Kerr-switching. Indeed, a high Kerr nonlinearity of $n_{2}=1.4 \cdot 10^{-2} \mathrm{~cm}^{2} \mathrm{GW}^{-1}$ was inferred based on the instantaneous transmission change [137]. This is around 2 orders of magnitude higher than the value determined via a z-scan $[133 ; 139]$ on bulk Si. In this Chapter, we will critically evaluate optical processes at both instantaneous ( $\mathrm{fs}$ ) as well as ps timescales. At coincidence, we will show that two competing processes change the optical properties as witnessed from reflectivity measurements. From these two processes, we deduce non-degenerate figure of merit (NFOM), which has wide implications for fs switching, extending to DOS switching and ultimately to the dynamic control of spontaneous emission.

\subsection{Experimental setup and sample}

We have discussed the experimental setup and procedure in detail in Chapter 2. Figure 5.1 shows a high resolution scanning electron micrograph of the photonic woodpile structure made by Jim Fleming at Sandia National Laboratories [38]. It consists of 5 layers of polysilicon rods $\left(n^{\prime}=3.45\right)$ stacked orthogonally upon one another, the n-th layer shifted by half an interrod distance with respect to the $(n+2)$ th layer. This structure gives rise to a diamond symmetry, for which a first-order band gap is predicted [12]. The dimensions of the rods were chosen so as to aim the center the band gap around telecom frequencies $\left(E_{\text {Tele }}=0.735 \mathrm{eV}\right)$. The E-field of the probe beam is polarized along the [110] direction of the crystal, that is, perpendicular to the first row of rods. The last row of rods is supported by a $70 \mathrm{~nm}$ thick SiN layer of refractive index $\approx 2$. At our probe wavelengths the SiN layer is optically thin, and is not expected to influence reflectivity measurements.

The sample has been characterized extensively elsewhere [95; 140]. Over 


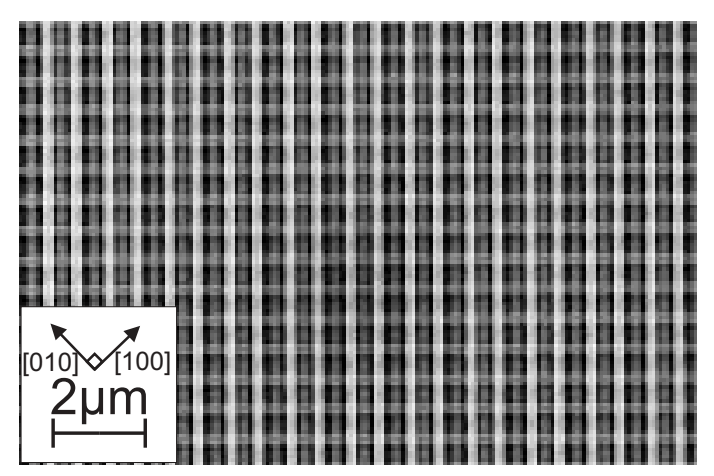

Figure 5.1.: High resolution scanning electron micrographs of the surface normal to [001] of a Si woodpile crystal at domain D4. The width and thickness of each rod is $175 \pm 10 \mathrm{~nm}$ and $155 \pm 10 \mathrm{~nm}$, respectively.

the sample, the mutual alignment of the rods differs. These differences are spatially separated, and allow the sample to be divided into 16 domains, which we denote by A1 through D4 in analogy to a chessboard. Here, we perform measurements on both $\mathrm{A} 1$ and D4, and are thus able to validate our results for different sample conditions. The symmetry of D4 is face centered orthorhombic, while A1 is body centered orthorhombic.

\subsection{Linear reflectivity}

Figure 5.2 shows a linear reflectivity spectrum of the woodpile photonic crystal. The high peak centered at $E_{0}=0.9 \mathrm{eV}$ corresponds to the $\Gamma-X$ stopgap in the band structure, which is part of the $3 \mathrm{D}$ photonic band gap of Si woodpile photonic crystals $[36 ; 96 ; 141]$. The maximum reflectivity of $95 \pm 2 \%$ and the broad width of $\Delta E=0.46 \mathrm{eV} \mathrm{FWHM} \mathrm{(full} \mathrm{width} \mathrm{at} \mathrm{half}$ maximum) confirm the strong interaction of the crystal with light [27]. We note that the large relative width of $47 \%$ is the largest measured for any photonic crystal so far. From the width and $E_{0}$, we deduce a Bragg length of $L_{B}=950 \mathrm{~nm}$ (equation 3 in the introduction), corresponding to 1.2 unit cells. In contrast, other $3 \mathrm{D}$ structures with a lower photonic strength have much higher $L_{B}$ : $\mathrm{TiO}_{2}$ inverse opals, for example, have $L_{B} \approx 4 \mu \mathrm{m}$, while PS opals have $L_{B} \approx 8 \mu \mathrm{m}$ and $\mathrm{SiO}_{2}$ opals $L_{B} \approx 9 \mu \mathrm{m}$ estimated from the bandstructure [26]. The high photonic strength also causes the steep edges of the peak, which we will in first instance probe at the blue edge of the stopband. At these frequencies, all induced changes in optical properties of the Si backbone will show a large change in reflectivity, important for 


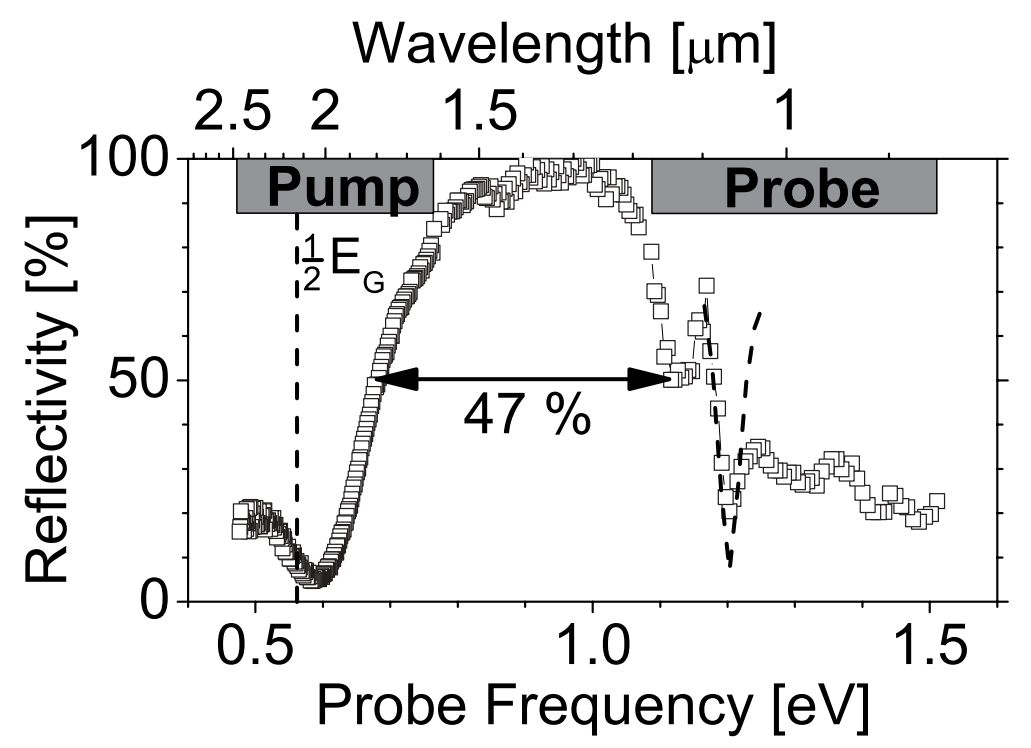

Figure 5.2.: Linear reflectivity spectrum of the woodpile photonic crystal measured normal to the [001] direction at a sample position D4 shown in figure 5.1. The $E$-field is perpendicular to the first row of rods. The $\Gamma-X$ stopgap at $0.9 \mathrm{eV}$ gives rise to a high maximum reflectivity of $95 \pm 2 \%$ and has a broad relative width of $47 \%$, indicating a high photonic strength. At high frequencies $>1.2 \mathrm{eV}$, the spectral features are attributed to Fabry-Pérot-type fringes. The pump frequencies (shaded box) were tuned at the red edge through half the electronic band gap $E_{\mathrm{G}}=1.12 \mathrm{eV}$ of silicon (vertical dashed line), and the probe frequencies at the blue edge of the stopband. The dashed curve is a calculation with the Scalar Wave Approximation in the region of interest.

switching applications. The probe frequencies (indicated by the shaded box) span both the blue edge of the stopband as well as the fringes. These have been assigned to Fabry-Pérot-type interferences [142], but the reflectivity spectrum of woodpiles is still subject to debate [95; 140].

\subsection{Switched reflectivity vs. delay at one probe frequency}

The pump frequencies $E_{\text {Pump }}$ were chosen as to tune through half the electronic band gap of silicon, where the gap is $E_{\mathrm{G}}=1.12 \mathrm{eV}$. Both the Kerr coefficient $n_{2}$ as the degenerate two-photon absorption coefficient $\beta_{11}$ have 
recently been shown to vary strongly in the vicinity of $\frac{1}{2} E_{G}[133-135 ; 143-$ 145].

On domain A1 we measured differential reflectivity $\Delta R / R$ vs. probe delay $\Delta t$ as a function of $E_{\mathrm{Pump}}$ (figure 5.3). At this sample position, the probe frequency of $E_{\text {Probe }}=1.13 \mathrm{eV}$ corresponds to the foot of the blue stopband edge. At positive probe delays $\Delta t=0.2 \mathrm{ps}$ and at pump frequencies $E_{\mathrm{Pump}}>$ $\frac{1}{2} E_{\mathrm{G}}=0.56 \mathrm{eV}$, we observe a large positive differential reflectivity $(\Delta R / R)_{\mathrm{FC}}$ due to a blue shift of the photonic features. This shift is caused by a decrease of the refractive index of of the polysilicon backbone that agrees well with a Drude description of the excited free carriers [96]. When $E_{\text {Pump }}$ is reduced to below $\frac{1}{2} E_{\mathrm{G}}$, the dispersion vanishes, since no more free carriers can be excited by a two-photon process.

Near coincidence, at $\Delta t=0 \mathrm{ps}$, a trough of magnitude $(\Delta R / R)_{\text {coinc }}$ is observed. The width of this trough varies between $240 \mathrm{fs}\left(E_{\mathrm{Pump}}=0.52 \mathrm{eV}\right)$ and $160 \mathrm{fs}\left(E_{\mathrm{Pump}}=0.62 \mathrm{eV}\right)$. The expected cross-correlation duration for two pulses of $\tau_{P}=140 \pm 10 \mathrm{fs}$ is $\tau_{P} \cdot \sqrt{2}=198 \mathrm{fs}$, in reasonable agreement to the measured values. The variation with pump frequency is partially related to the shorter pulse duration at higher frequencies which corresponds to a wider spectral width of the pulse. As $E_{\text {Pump }}$ increases to above $\frac{1}{2} E_{\mathrm{G}}$, the magnitude of $(\Delta R / R)_{\text {coinc }}$ grows until it saturates at $E_{\text {Pump }}=0.62 \mathrm{eV}$, just over $\frac{1}{2} E_{\mathrm{G}}$.

\subsection{Switched reflectivity vs. frequency}

\subsubsection{Reflectivity vs. frequency at coincidence}

In order to investigate the precise nature of $(\Delta R / R)_{\text {coinc }}$, we have measured the differential reflectivity at coincidence for a range of probe frequencies (figure $5.4(\mathrm{c})$ ) on domain D4. With increasing $E_{\mathrm{Pump}}$, the variation in the data becomes stronger. The differential reflectivity becomes increasingly negative with both increasing pump and probe frequency, indicating optical absorption. At $1.22 \mathrm{eV}$, a peak appears in the differential reflectivity that corresponds to a red edge in the linear reflectivity (figure 5.4(a)) and at $1.18 \mathrm{eV}$ at trough appears which corresponds to a blue edge. These two observations are indicative of a red shift of the photonic features. Therefore, when pump and probe are coincident, two effects contribute to a negative differential reflectivity at blue edges of photonic features: Non-linear dispersion, which we attribute to the electronic Kerr effect, and absorption. Since the latter only occurs in the presence of the pump, we conclude that the 


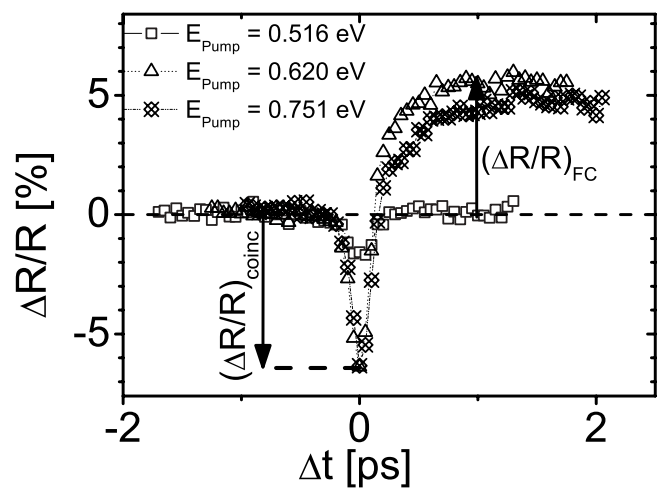

Figure 5.3.: Differential reflectivity $\Delta R / R$ versus probe delay $\Delta t$ taken at different pump frequencies $E_{\text {Pump }}$ and at probe frequency $E_{\text {Probe }}=1.13 \mathrm{eV}$, sample position A1. At $\Delta t=0 \mathrm{ps}$, the pump and probe are coincident, and the differential reflectivity $(\Delta R / R)_{\text {coinc }}$ decreases. At $\Delta t=1 \mathrm{ps}$, the differential reflectivity $(\Delta R / R)_{\mathrm{FC}}$ has increased due to the dispersion of the free carriers $(F C)$. The peak pump irradiance varies between $I_{\text {Pump }}=10 \pm 1 \mathrm{GWcm}^{-2}\left(E_{\mathrm{Pump}}=0.516 \mathrm{eV}\right)$ and $I_{\mathrm{Pump}}=25 \pm 2$ $G W \mathrm{Cm}^{-2}\left(E_{\mathrm{Pump}}=0.75 \mathrm{eV}\right)$, and the probe irradiance was $I_{\text {Probe }}=3 \pm 2 \mathrm{GWcm}^{-2}$.

negative differential reflectivity is due to an instantaneous non-degenerate two-photon process. This conclusion is consistent with the observation that at higher probe frequencies, for any given pump frequency, the negative offset increases. Recent work [136-138] has attributed the behavior at coincidence to the Kerr effect, due to the lack of dispersive data. We for the first time identify the different contributions at coincidence.

\subsubsection{Reflectivity vs. frequency at $\Delta t=1$ ps}

Figure 5.4(b) shows the differential reflectivity at one positive probe delay of $\Delta t=1 \mathrm{ps}$ that is caused by free carriers. Because the data varies around 0 , we conclude that the induced absorption is minimal. The peak at 1.2 $\mathrm{eV}$ corresponds to the blue edge of the linear reflectivity peak at $1.175 \mathrm{eV}$, the two troughs at $1.23 \mathrm{eV}$ and $1.16 \mathrm{eV}$ corresponds to the red edges of the reflectivity peaks at 1.175 and $1.25 \mathrm{eV}$, respectively. Therefore, the data is consistent with a blue shift, or a decrease of the refractive index $\Delta n^{\prime}$ as a result of the free carriers. 


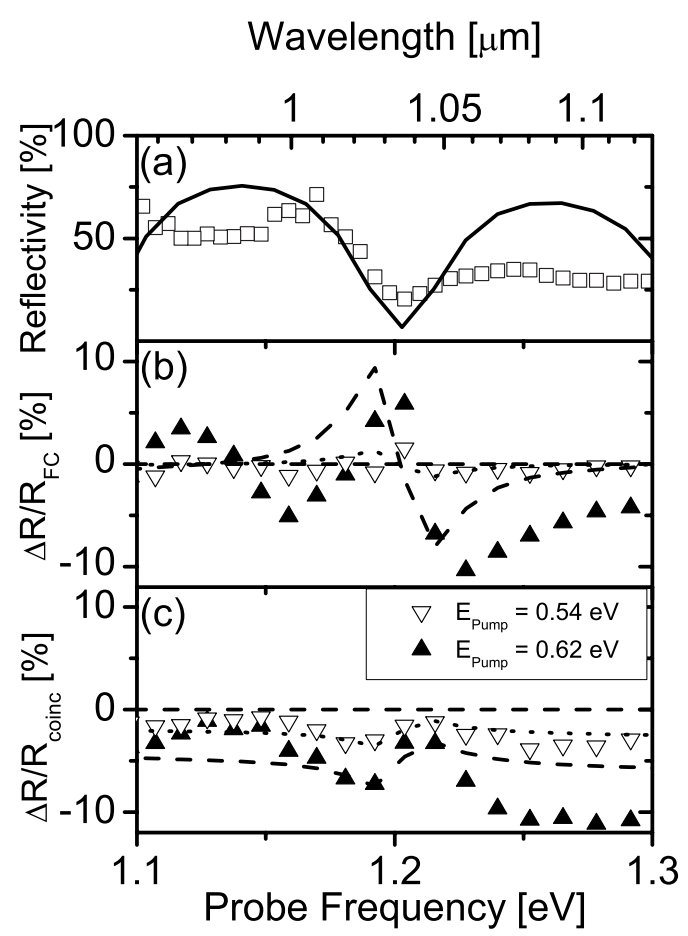

Figure 5.4: (a): Measured linear reflectivity versus probe frequency $(\square)$ on domain D4 compared to a scalar two-band model (solid curve). (b) The differential reflectivity at delay $\Delta t=1 \mathrm{ps}$ caused by the Free Carriers $(\Delta R / R)_{\mathrm{FC}}$ shows mostly dispersive features, as seen from the symmetric variation of $\Delta R / R$ around 0 . Dashed and dotted lines correspond to the fits of the extended SWA at $E_{\text {Pump }}=0.54$ and $0.62 \mathrm{eV}$, respectively. (c): Dispersive as well as absorptive elements can be observed in $\Delta R / R_{\text {coinc }}$, increasingly so when increasing the pump frequency from $E_{\mathrm{Pump}}=$ $0.54 \mathrm{eV}$ (black triangles) to $E_{\mathrm{Pump}}=0.62$ $\mathrm{eV}$. The pump irradiance was $I_{\mathrm{Pump}}=$ $36 \pm 4 \mathrm{GWcm}^{-2}\left(E_{\text {Pump }}=0.54 \mathrm{eV}\right)$ and $I_{\text {Pump }}=46 \pm 5 G_{C m^{-2}}\left(E_{\mathrm{Pump}}=0.62\right.$ $\mathrm{eV})$.

\subsubsection{Model: the extended scalar wave approximation}

The optical properties of real woodpile photonic crystals are usually calculated with an exact modal method (EMM) [141]. For each layer in $z$, the dielectric constant $\epsilon(\mathbf{r})$ is expanded in Fourier series in $x$ and $y$, perpendicular to the $z$. The electric and magnetic fields can be calculated from the vector wave equation. The solutions are then subject to boundary conditions from the solutions in adjacent layers. In that way, the fields in the complete structure can be calculated. It is different to a bandstructure calculation in that the structure is finite in one direction. From the boundary conditions at the first and the last layer, it is possible to calculate the reflectivity. The EMM has been shown to agree reasonably well to reflectivity measurements at frequencies up to the stopgap [95; 96; 142], but fails to reproduce measurements at the blue edge of the stopgap. If we quantitatively want to understand the magnitude of the induced changes, it is important for any model to correctly reproduce the linear reflectivity first.

The Scalar Wave Approximation (SWA) turns the vector wave equation, resulting from Maxwell's equations, into a scalar equation, where the scalar is in the beam direction $z$ [146]. Because of the periodicity of the lattice, both the real lattice spacings and the position dependent dielectric function 
$\epsilon(z)$ can be transformed into Fourier Space, with Fourier Transforms $G$ and $U_{G}$ respectively. For binary structures composed of materials of two different dielectric constants, it can be shown that [26]

$$
U_{G}=\Delta \epsilon f_{G}, G \neq 0,
$$

where $\Delta \epsilon$ is the difference of the dielectric constants, and $f_{G}$ is the Fourier Transform of the indicator function $f(z)$, where $\epsilon(z)=\epsilon_{1}+\Delta \epsilon f(z)$. By satisfying field continuity of the two bands labelled $k$ (incident) and $k-G$ (Bragg diffracted) at the two interfaces of the crystal, the transmission and reflectivity can be determined.

Two remarks are at order: a. Despite evidence of polarization effects on the reflectivity [142], the scalar wave approximation inherently cannot take polarization into account. However, since the incident polarization does not change during the experiment, the induced change in reflectivity can just as well be modelled with a scalar equation. b. While EMM can correctly include absorption by solving Maxwell's equations with a complex $\epsilon$ in any one layer, the SWA uses an approximated two-band bandstructure, for which modes can only exist in the absence of extinction. To nevertheless include absorption, the reflectivity was reduced by a factor $\exp \left(-2 L / \ell_{\text {abs }}\right)$, where $\ell_{\mathrm{abs}}=1 /\left(2 n^{\prime \prime} n^{\prime} k\right)$. The factor of 2 in the exponent accounts for the beam passing through the sample twice. This results in the magnitude of the reflectivity over all the spectrum to decrease, while induced absorption, such as non-degenerate absorption as in our case, normally causes the visibility to decrease, due to the increasing breakdown in interference. In view of this second point, the fitted values of $n^{\prime}$ and $n^{\prime \prime}$ must be regarded as qualitative.

We fit the linear reflectivity obtained by the scalar wave approximation to the region given in fig. 5.4(a). The best agreement to the measured reflectivity is obtained with $G=2 \pi /(393 \mathrm{~nm})$ and $U_{G}=-0.8$. Due to the scalar nature of the model, we expect no correlation between the real and fitted lattice constants. In the model, we included the dispersion and absorption of p-Si (from [147], see fig. 5.5), and have taken bulk $\mathrm{Si}$ as the substrate, neglecting the optically thin layer of $\operatorname{SiN}\left(n^{\prime}=2, d=70 \mathrm{~nm}\right)$. Since the optical features in the reflectivity spectrum are much broader than the probe bandwidth, no convolution of the calculated spectrum with the probe is necessary. 


\subsubsection{Interpretation of spectra at coincidence}

We match the calculated dispersive differential reflectivity to the strong signature of a redshift at $E_{\text {Probe }}=1.2 \mathrm{eV}$ by fitting two independent parameters $\Delta n^{\prime}=n_{2} I_{\text {Pump }}$ and $n^{\prime \prime}$ over the range $1.18 \mathrm{eV}<E_{\text {Probe }}<1.23 \mathrm{eV}$ by optimizing for least squares. The differential reflectivity was obtained as follows: using the $G$ obtained previously from the fit to the linear spectrum, the $U_{G}$ was changed via the changed $\Delta \epsilon$ (equation 5.1 ), and the switched reflectivity spectrum was calculated from the new $U_{G}$. The reduced $\chi^{2}$, i.e., the sum of the squared differences divided by the degrees of freedom, was calculated to be between 0.29 and 0.98. Error margins for the parameters were taken as the values at which the least squares had changed by $10 \%$.

Figure 5.4(c) shows the fits to two spectra obtained at two different $E_{\text {Pump }}$. The remarkable agreement of this approximate model with the data confirms that the subtle interplay of non-linear dispersion as well as non-degenerate absorption leads to the observed features. At frequencies lower than $1.2 \mathrm{eV}$, the fitted curves tend to be lower than the measurements. Also, to the blue of $1.2 \mathrm{eV}$, the calculated differential reflectivity is mostly higher than the measurements. These deviations are due to our fit not taking into account the non-degenerate nature of $\beta_{12}$, but setting it to one value for all probe frequencies.

Figure 5.5(a) and (b) shows the absorptive and dispersive part of the refractive index, respectively, as returned from the fits. The value at $E_{\text {Probe }}=$ $0.7 \mathrm{eV}$ is excluded due to experimental artifact in that measurement. Figure 5.5(a) shows that the imaginary refractive index $n^{\prime \prime}$ increases for pump frequencies $E_{\text {Pump }}>0.55 \mathrm{eV}$. Since we are working at pump-probe coincidence, those frequencies correspond to a sum of pump and probe frequencies $E_{\text {Pump }}+E_{\text {Probe }}>1.75 \mathrm{eV}$. Since these values are larger than the the optical gap of polycrystalline $\mathrm{Si}$ of $1.4 \mathrm{eV}<E_{\mathrm{opt}}<1.6 \mathrm{eV}$, depending on the degree of polycrystallinity [148], it is clear that the simultaneous presence of pump and probe photons induce optical absorption. From recent calculations [149], the N-photon process in a direct transition can be viewed as a single-photon process with an energy gap rescaled to $E_{G} / N_{\mathrm{ph}}$, where $N_{\mathrm{ph}}$ is the number of photons. In other words, $\left(E_{\text {Pump }}+E_{\text {Probe }}\right) / 2=E^{\prime}$ in the two beam case, where $E^{\prime}$ is a rescaled energy. Therefore, we can compare the shape of our extracted non-degenerate absorption coefficient to the linear absorption. We therefore also plot the linear absorption of p-Si (LPCVD, processing temperature $545^{\circ} \mathrm{C}$ ) similar to that used to make the woodpile crystals [147]. The behavior of the linear absorption agrees reasonably with 
that of the non-degenerate absorption, supporting calculations from [149]. For our intensities, the non-degenerate absorption remains below the linear absorption by a factor of 35 . Pumping and/or probing at higher frequencies causes the absorption to increase, as do higher pump intensities.

Higher probe intensities do not influence the magnitude of the nondegenerate absorption: in the absence of linear absorption, as in our case, the differential equation governing non-degenerate two photon absorption is [150]

$$
\frac{d I_{\text {Probe }}}{d z}=-2 \beta_{12}\left(E_{\text {Probe }}, E_{\text {Pump }}\right) I_{\text {Pump }} I_{\text {Probe }}(z)
$$

The non-degenerate two-photon absorption coefficient $\beta_{12}$ is related to $n^{\prime \prime}$ as $\beta_{12} I_{\text {Pump }}=2 n^{\prime \prime} n^{\prime} k$. From equation 5.2 we see that higher probe intensities do not increase absorption: The coefficient of $-I_{\text {Probe }}(z)$ is $2 \beta_{12}\left(E_{\text {Probe }}+\right.$ $\left.E_{\text {Pump }}\right) I_{\text {Pump }}=\alpha_{\text {eff }}$, an effective absorption coefficient. Higher probe intensities merely lead to a higher absorbance, commensurate to the number of absorbed photons.

The real part of the instantaneous change in $n$ is plotted in figure 5.5(b). At low pump frequencies of $0.52 \mathrm{eV}$, the change is $0.5 \cdot 10^{-3}$, while it more than doubles at $E_{\text {Pump }}=0.56 \mathrm{eV}$. At $E_{\text {Pump }}=0.62 \mathrm{eV}, \Delta n^{\prime}$ increases only marginally. We compare our measurements to other recent degenerate measurements of the nonlinear dispersion of bulk $\mathrm{Si}$ [133-135]. In order to be able to make the comparison, we made use of the rescaling condition whose validity is unknown for dispersion. We find excellent agreement to the latter, but surprisingly the measurements of [133] are an order of magnitude higher, despite being measured with the same technique. We also compare our measured data to a relation for $n_{2}$ derived for direct-gap semiconductors [151] multiplied by our pump irradiance $I_{\text {Pump }}$. The functional form agrees well with our data. At higher probe frequencies, the theoretical relation differs from the data of $[134 ; 135]$. A theory able to calculate the degenerate nonlinear properties of an indirect semiconductor such as silicon was recently proposed [149] and was shown to agree well with degenerate nonlinearites [133]. 


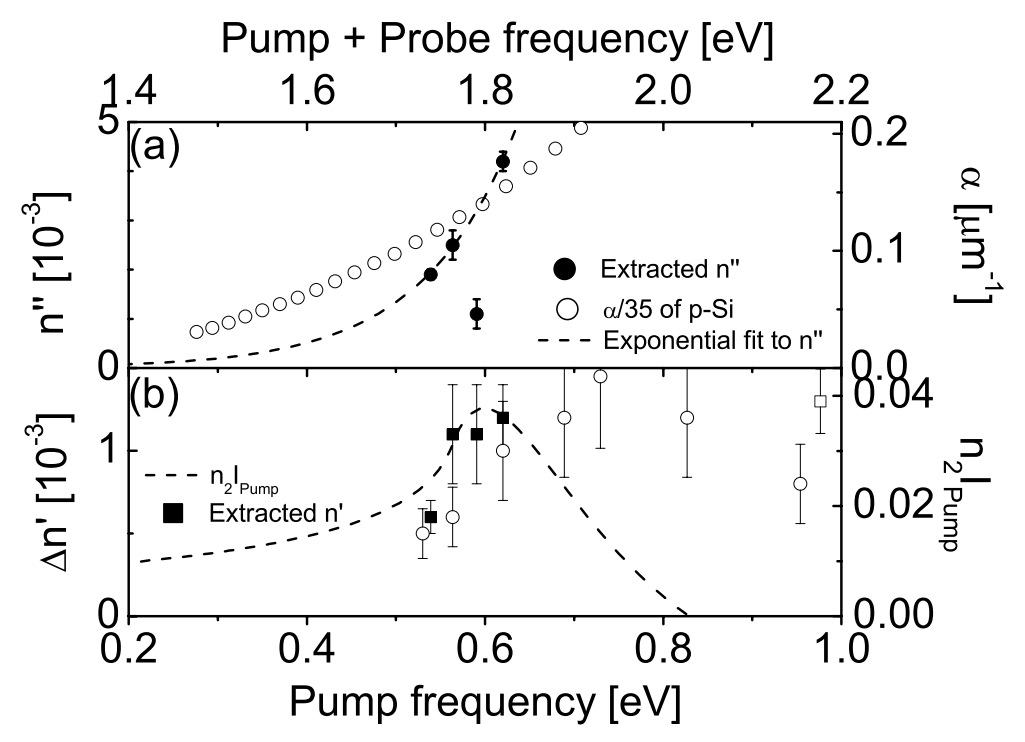

Figure 5.5.: (a): Imaginary refractive index ( $\left.n^{\prime \prime}, \bullet\right)$ vs. pump frequency $E_{\text {Pump }}$ (lower axis) obtained by modeling our data (fig. 5.4) and upper abcissa is the summed pump and probe frequencies $E_{\text {Total }}$. The right scale shows the corresponding absorption coefficient. To compare to our effective $\alpha$, we plot the absorption coefficient for LPVCD p-Si (O) annealed at $545^{O}$ C, similar to the backbone of our woodpiles crystals. (b): Change in real refractive index $\Delta n^{\prime}$ (left scale, $\mathbf{\square}$ ) vs. pump frequency $E_{\mathrm{Pump}}$ from our non-degenerate measurements. We plot other recent degenerate measurements for comparison: $\square$ from [135] and $\bigcirc$ from [134]. Data from [133] exceeds the scale by one order of magnitude. The theoretical relation predicted by [151] is also shown (solid curve, right scale). 


\subsubsection{Interpretation of ps switching}

Using the same two-band model from section 5.5.3, we can also interpret the picosecond free-carrier behavior. Therefore, the calculated differential reflectivity can be fitted to $(\Delta R / R)_{\mathrm{FC}}$ by calculating the complex refractive change according to the Drude model (figure 5.4(b)). For carrier densities $N<10^{28} \mathrm{~m}^{-3}$, well above densities created in our experiments, $N$ is given by $[73]$

$$
N=\frac{I_{\text {Pump }}^{2} \tau_{P} \beta_{11}}{2 E_{\text {Pump }} e},
$$

where $\beta_{11}$ is the degenerate two photon absorption coefficient. At our pump frequencies, we can neglect linear absorption. Then, the change in dielectric constant due to free carriers is

$$
\epsilon_{\mathrm{fc}}=-\frac{\omega_{P}^{2}}{\omega^{2}}\left(1-i\left(\omega \tau_{D}\right)^{-1}\right),
$$

where $\omega_{P}^{2}=N e^{2} / m^{*} \epsilon_{0}$ is the plasma frequency squared in $(\mathrm{rad} / \mathrm{s})^{2}$ and $e$ and $m^{*}$ are the charge and effective optical mass of the carriers, and $\omega$ the probe frequency in $\mathrm{rad} / \mathrm{s}$. Equation 5.4 is valid for $\left(\omega \tau_{D}\right)^{-1}<<1$, which is the case for our probe frequencies and damping times. For a given $I_{\mathrm{Pump}}$ therefore, we can fit the differential reflectivity resulting from a changing $\beta_{11}$ to $(\Delta R / R)_{\mathrm{FC}}$.

The $\beta_{11}$ vs. $E_{\text {Pump }}$ as returned from the fits is plotted in figure 5.6. We find reduced $\chi^{2}$ values between 5 and 25 . These values are higher than the reduced $\chi^{2}$ from the fits at coincidence, mainly because of a shift in spectral features of the calculated spectrum, a behavior which is not well understood. As $E_{\text {Pump }}$ decreases to below $\frac{1}{2} E_{G}, \beta_{11}$ vanishes, as expected. Increasing $E_{\text {Pump }}$ to above $0.54 \mathrm{eV}$, there is a marked increase. At $E_{\text {Pump }}=0.62 \mathrm{eV}, \beta_{11}$ has increased to as much as $5 \pm 1 \mathrm{cmGW}^{-1}$. This value is considerably higher than other published values [133-135], measured with the z-scan technique on crystalline Si. For our sample however, values as high as $\beta_{11}=60 \pm 15$ $\mathrm{cmGW}^{-1}$ have been reported [95]. Amorphous silicon formed by CVD has very recently been shown to posses a higher non-linearity [152], in agreement to our data.

We are now able to calculate some parameters describing the switching quality. We find that the carrier induced absorption length $\ell_{\mathrm{abs}}=123 \pm$ $50 \mu \mathrm{m}$, much longer than $L_{B}$, confirming that the absorption is negligible indeed. The pump homogeneity length, another important parameter describing the spatial homogeneity of carriers, is $\ell_{\mathrm{hom}}=1 / I_{\mathrm{Pump}} \beta_{11}=44 \pm 10 \mu \mathrm{m}$, 


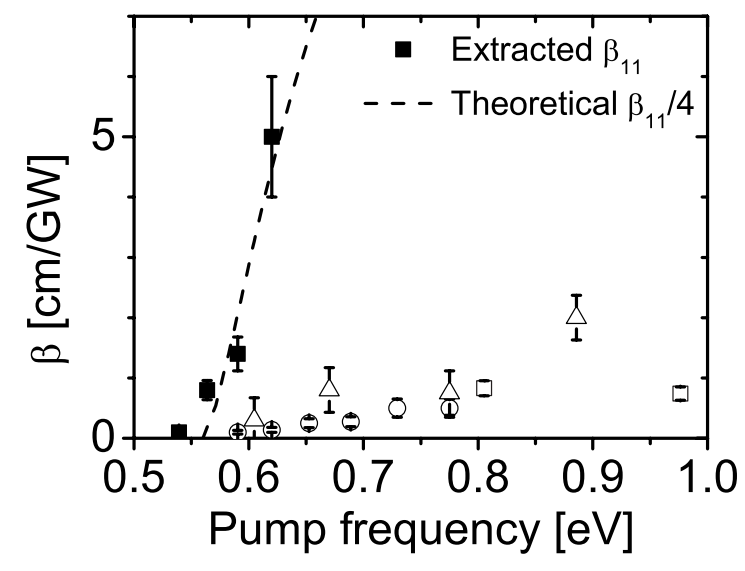

Figure 5.6: $\beta_{11}$ vs. pump frequency (ם). We fit the differential reflectivity obtained from the two-band model to the data with $\beta_{11}$ as the only free parameter. Other data are from $\square([135]), \bigcirc([134])$, and $\triangle$ $([133])$.

or $170 \pm 40 L_{B}$ at $E_{\text {Pump }}=0.62 \mathrm{eV}$. This calculation highlights the advantage of two-photon absorption to one-photon absorption $[102 ; 136 ; 138]$ in which absorption limits the pump homogeneity. In contrast, the absorption length of the nondegenerate two-photon process is $\approx 10 \mu \mathrm{m}$.

\subsection{Reflectivity vs. pump frequency at coincidence}

Having analyzed the reflectivity spectra at coincidence and at $\Delta t=1 \mathrm{ps}$, we return to figure 5.3, where we had measured the differential reflectivity vs. probe delay at only one probe frequency, but at many pump frequencies. Experimental conditions had prevented us from taking more than 4 probe frequency- and time-resolved spectra, mostly related to the long measurement times. Having identified two competing processes at coincidence, we can therefore now focus on the time-resolved differential reflectivity, which we had measured at many pump frequencies. Figure 5.7 shows $(\Delta R / R)_{\text {coinc }}$ at different $E_{\text {Pump }}$. Here, $I_{\text {Pump }}$ has been corrected for three important pump beam parameters: i., the increase in irradiance with increasing $E_{\text {Pump }}$ because of the decreasing Gaussian focus (see 2.2); ii. the change in pump irradiance due to the frequency dependent reflectivity of the pump, see figure 5.2 ; and iii. the measured $10 \%$ change in pump power over the frequency range of the Topas. For i., $(\Delta R / R)_{\text {coinc }}$ is assumed to vary quadratically with $E_{\text {Pump }}$ (fig. 2.2), assuming that $(\Delta R / R)_{\text {coinc }}$ changes linearly with $I_{\text {Pump }}[140]$. For (ii), the pump reflectivity was treated as a Fresnel reflection and thus $(\Delta R / R)_{\text {coinc }}$ was linearly corrected with $\left(1-R\left(E_{\text {Pump }}\right)\right)$ in b. Finally, in iii., we corrected $(\Delta R / R)_{\text {coinc }}$ linearly with $I_{\text {Pump }}$. The two highest corrections are by $80 \%\left(E_{\text {Pump }}=0.52 \mathrm{eV}\right)$ and $34 \%\left(E_{\mathrm{Pump}}=0.56\right.$ 
$\mathrm{eV})$, but otherwise the corrections are less than $20 \%$.

In figure 5.7 we observe an increasing $(\Delta R / R)_{\text {coinc }}$ with increasing $E_{\text {Pump }}$ up to $0.62 \mathrm{eV}$, then a decrease until $0.69 \mathrm{eV}$ before a subsequent increase. With the analysis of the previous data (fig. 5.5), two important regimes can now be identified: for $E_{\text {Pump }}<0.69 \mathrm{eV}\left(E_{\text {Total }}<1.82 \mathrm{eV}\right),(\Delta R / R)_{\text {coinc }}$ is mostly governed by dispersion. For $E_{\mathrm{Pump}}>0.69 \mathrm{eV},(\Delta R / R)_{\text {coinc }}$ increases again due to a combination of both dispersive $\left(n_{2}\right)$ as well as absorptive parts $\left(\beta_{12}\right)$.

In the same figure 5.7, the differential reflectivity expected from the extended SWA is also plotted. Because this different reflectivity spectrum was obtained on domain A1, we first have to apply the SWA to the linear reflectivity from A1 (not shown). We obtain best agreement for $G=2 \pi / 363 \mu \mathrm{m}$ and $U_{G}=-1.2$. From the theoretical relation for a degenerate $n_{2}$ of a direct bandgap semiconductor [151], $(\Delta R / R)_{\text {coinc }}$ is readily obtained. We note that a non-degenerate theory for indirect bandgap semiconductors is not available. We therefore have to interpret the measurements with respect to the theoretical relation cautiously. For $E_{\text {Pump }}<0.7 \mathrm{eV}$, the shape of the theoretical relation agrees excellently to our measurements. A strong divergence from the data is found for $E_{\text {Pump }}>0.7 \mathrm{eV}$, or $E_{\text {Total }}>1.83 \mathrm{eV}$. We interpret this threshold frequency marking a transition from dispersive behavior of $(\Delta R / R)_{\text {coinc }}$ to an absorptive behavior.

To verify the consistency of the results of sections 5.5.1 and 5.5.2 with the previous ones, both $n_{2}$ and $\beta_{12}$ (figure 5.5) were inserted into the SWA, and plotted in figure 5.7. Good agreement to the measured differential reflectivity is found, from which we conclude that the extended SWA provides consistent description of our various data, measured at different pump intensities and on sample domains.

\subsection{Non-degenerate instantaneous figure of merit}

To quantify how useful a switching device is, it is instructive to consider a figure of merit (FOM). Garmire proposed that the device length be longer than the pump absorption length, for which the corresponding pump irradiance would induce a phase shift of $2 \pi f$. From this requirement a figure of merit has been derived [153],

$$
\operatorname{FOM}\left(E_{\text {Pump }}\right)=f=n_{2} / \lambda \beta_{11} .
$$

For propagation switching, it is necessary that $\mathrm{FOM}=\frac{1}{2}$. The notion of a FOM can be extended to include non-degenerate absorption. Not only is the 


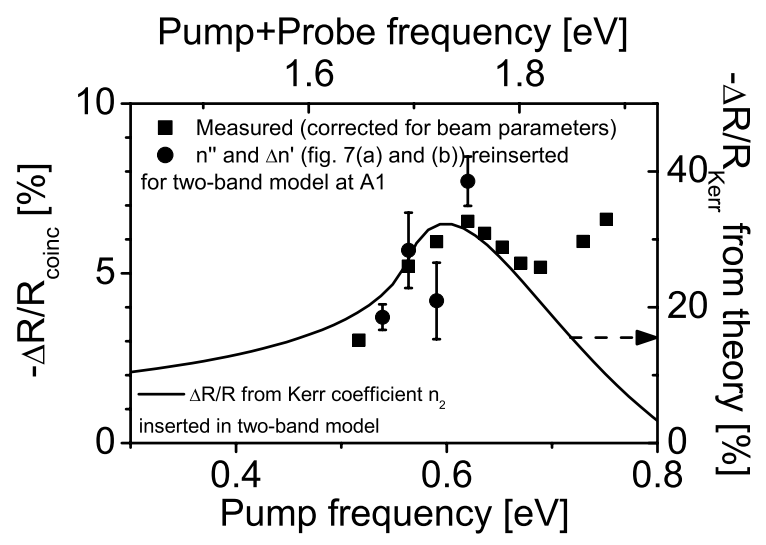

Figure 5.7.: Differential reflectivity at coincidence ( $\mathbf{\square}$, left scale) vs. pump frequency extracted from figure 5.3 and corrected for pump beam parameters. We have calculated the differential reflectivity expected from a non-linearly purely dispersive direct bandgap material [151] by inserting the theoretical relation for $n_{2}$ into the SWA. We can clearly see the 'pure dispersive assumption' is wrong as from $E_{\text {Pump }}=0.69 \mathrm{eV}$, or $E_{\text {Total }}=1.82 \mathrm{eV}$. Extracted complex values of $n$ (from figure 5.5) have been re-inserted into this model. Reasonable agreement to the measurements $\mathbf{\text { is found. }}$

pump absorbed because it excites free carriers, but the probe is absorbed in the presence of the pump. The nondegenerate FOM (NFOM) is then

$$
\operatorname{NFOM}\left(\mathrm{E}_{\text {Pump }}, \mathrm{E}_{\text {Probe }}\right)=\frac{\mathrm{n}_{2}\left(\mathrm{E}_{\text {Pump }}\right)}{\lambda_{\text {Pump }}\left(\beta_{11}\left(\mathrm{E}_{\text {Pump }}\right)\right)+\beta_{12}\left(\mathrm{E}_{\text {Pump }}, \mathrm{E}_{\text {Probe }}\right)} .
$$

From our measured data, the NFOM has been calculated as follows: $n_{2}$, where $n_{2}=\left(E_{\text {Pump }}\right)$ has been derived from figure 5.5(b), where we have used the functional form of $n_{2}\left(E_{\mathrm{Pump}}\right)$ from [151], but scaled it by a constant factor to match the magnitude of our data. A analytic form of $\beta_{12}$ was derived by fitting an exponential to linear absorption coefficient $\alpha\left(E_{\text {Pump }}+E_{\text {Probe }}\right)$. The exponential behavior of the linear absorption coefficient $\alpha$ with $E_{\text {Probe }}$ close to the gap region is well documented [154]. From $\alpha\left(E_{\text {Pump }}+E_{\text {Probe }}\right)$, we deduce $\beta_{12}=\alpha\left(E_{\text {Pump }}+E_{\text {Probe }}\right) / 2 I_{\text {Pump }}$ from equation 5.2. Finally, an analytic expression for $\beta_{11}\left(E_{\text {Pump }}\right)$ is obtained from the theoretical relation for $\beta_{11}$ ( $E_{\text {Pump }}$ for direct bandgap semiconductors from [151], but again scaled by one factor to match our measurements (fig. 5.6).

Figure 5.8 shows the NFOM for different $E_{\text {Pump }}$ and $E_{\text {Probe }}$. At summed frequencies $E_{\text {Total }}=E_{\text {Pump }}+E_{\text {Probe }}$, below the optical gap silicon $\left(E_{\text {Total }}<\right.$ 


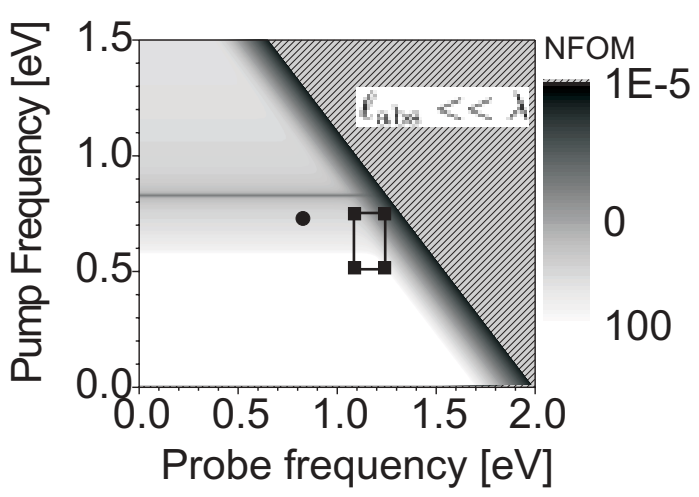

Figure 5.8.: Nondegenerate Figure of Merit (NFOM) for instantaneous switching vs. pump and probe frequency for silicon (see eq. 5.6). For $E_{\mathrm{Pump}}<\frac{1}{2} E_{G}$, pump absorption is absent as we do not consider higher order photon absorption. If in addition $E_{\text {Probe }}<E_{\text {Opt }}$ (white area), the total absorption is 0 leading to large NFOM outside of the scale of graph. Cross-sections of higher order photon absorption are required to calculate the NFOM at $E_{\text {Probe }}<E_{\text {Opt }}$ and $E_{\text {Pump }}<$ $\frac{1}{2} E_{G}$. The horizontal line at $E_{\mathrm{Pump}}=0.8 \mathrm{eV}$ marks the region where $n_{2}\left(E_{\mathrm{Pump}}\right)=0$ $\mathrm{cm}^{2} G W^{-2}$, and so NFOM $=0$. For $E_{\text {Total }}>E_{\mathrm{Opt}}$, the absorption length is shorter than one wavelength (hatched area). The parameter space from our measurements are bounded by the box $(\mathbf{\square})$, the only other measurement of $n_{2}$ on a Si based photonic crystal is [137] (•)

$\left.E_{\text {Opt }}\right)$, the non-degenerate two-photon absorption is small, tantamount to a long probe absorption length, which is desired. If in addition $E_{\text {Pump }}<$ $1 / 2 E_{G}$, the pump absorption length becomes infinite. Higher order photon absorption has to be considered to give a quantitative estimate of the NFOM in this regime. Around $E_{\text {Pump }}=0.8 \mathrm{eV}, n_{2}$ crosses the abscissa and the NFOM becomes 0 . For all other frequencies, NFOM depends strongly on both pump and probe frequencies. To achieve a high NFOM, it is advisable for $E_{\text {Total }}<E_{\text {Opt }}$ and $E_{\text {Pump }} \approx \frac{1}{2} E_{G}$. In that case, $n_{2}$ is high, while $\beta_{11}$ is kept low, and thus pump homogeneity can be vouchsafed. Our measurements have taken place in the parameter space indicated by the box. The only other measurement of $n_{2}$ on a Si based photonic crystal is [137].

\subsection{Conclusions and recommendations}

We have switched a silicon woodpile photonic woodpile crystal optically by both Kerr nonlinearities as well as optical free carrier injection. For the latter we achieve refractive index changes of up to $0.1 \%$ and changes in reflectivity of up to $\Delta R=1 \%$. At pump coincidence, we find that for 
$E_{\text {Total }}<1.8 \mathrm{eV}$, the reflectivity changes are due to the Kerr nonlinearity. In this case, the probe absorption length is limited by the pump power. For $E_{\text {Total }}>1.8 \mathrm{eV}$, the reflectivity starts to be dominated by pump-assisted absorption. For lower $E_{\text {Total }}$, it seems feasible to switch via the Kerr effect. Spontaneously emitted photons from an emitter placed inside the crystal are then amenable to switching. We have extended the figure of merit for Kerr switching to include nondegenerate two-photon absorption. For TiSaph lasers used in several previous experiments, $(E=1.55 \mathrm{eV})$, the NFOM is forbidding.

For future experiments, some technical issues are proposed. First, knowledge of the linear optical properties of bulk p-Si is necessary if its nonlinear properties are to be determined precisely. Neither $E_{\text {opt }}$ nor $n^{\prime}, n^{\prime \prime}$ were known for this sample, so these were essentially unknown parameters inserted into the extended SWA model. Furthermore, the degenerate and non-degenerate non-linear optical properties of bulk p-Si should be measured. This facilitates comparison to the same properties measured on a photonic bandgap crystal. Furthermore, we propose to validate the extended scalar wave model to the exact modal method, where responses of nonlinearities are compared at frequencies exhibiting similar spectral properties. Inspite of the difficulties in analyzing non-degenerate optical properties of a photonic bandgap crystal, we have measured a clear difference in response of $E_{\text {Total }}<E_{\text {Opt }}$ and $E_{\text {Total }}>E_{\mathrm{Opt}}$. This difference has ramifications on future experiments on non-degenerate instantaneous switching photonic bandgap crystals. DOS switching, and ultimately spontaneous emission switching is limited by the magnitude of pump and emission frequencies.

To exploit Kerr switching, that is to maximize NFOM, following improvements are proposed. In first instance it seems useful to pump and probe with the same polarization. For collinear polarization, a factor 3 improvement is predicted with respect to orthogonal polarization [155]. Furthermore, the optical properties of the photonic crystal could be so tailored as to enhance the field. Calculations by us on a 1D DBR have revealed that the field can significantly be enhanced when pumping at either the red or blue stopgap edge, while the position of the field maximum depends critically on the exact pump frequency.

While a high-bandgap semiconductor will be less prone to non-degenerate absorption, $n_{2}$ scales with $E_{G}^{-3}$. The absorption can also be diminished by a lower pump irradiance, which also goes at the expense of $\Delta n=n_{2} I_{\mathrm{Pump}}$. We therefore conclude that many obstacles will have to be overcome before Kerr switching can usefully be exploited. 



\section{Enhancement and reduction of an opal's photonic strength by atomic dispersion}

\subsection{Introduction}

\subsubsection{Atoms in photonic crystals}

The interplay between atomic and photonic resonances has recently lead to a surge of interest in the quantum optics community. From Fermi's golden rule it can be calculated that the spontaneous emission rate of an atom is no immutable property, but that it depends on the electromagnetic environment [156; 157]. Decay properties of atoms have been studied near interfaces [158], gratings [159], waveguides [160], fibers [161], and photonic crystals $[17 ; 19 ; 91]$. Modification of the decay rate becomes especially prominent when the atoms are in high-Q cavities $[162 ; 163]$ : at nodes of the cavity, the emission is enhanced by the Purcell factor [99], which depends on the quality factor $Q$ of the cavity. For very high-Q cavities, the atom and cavity couple strongly, and the atom-cavity system like a pair of coupled oscillators [164-168], evidenced by the avoided crossing of the two resonances.

Not only can the electromagnetic environment influence the decay rate of the atoms, but under given circumstances the atoms can alter the photonic properties of the host medium such as a photonic crystal [169]. The atom-photonic crystal system offers exciting prospects, such as creation of new modes in the photonic crystal's bandgap, induced transparency, nonlinearities and modified pulse propagation $[61 ; 170 ; 171]$. To achieve this, an ensemble of many atoms have to be placed in the crystal. The relative dielectric function $\epsilon(\omega)=\epsilon^{\prime}(\omega)+\epsilon^{\prime \prime}(\omega)$ of the atomic medium changes drastically around a resonance, $\epsilon^{\prime}(\omega)$ exceeding unity below resonance, and being below 


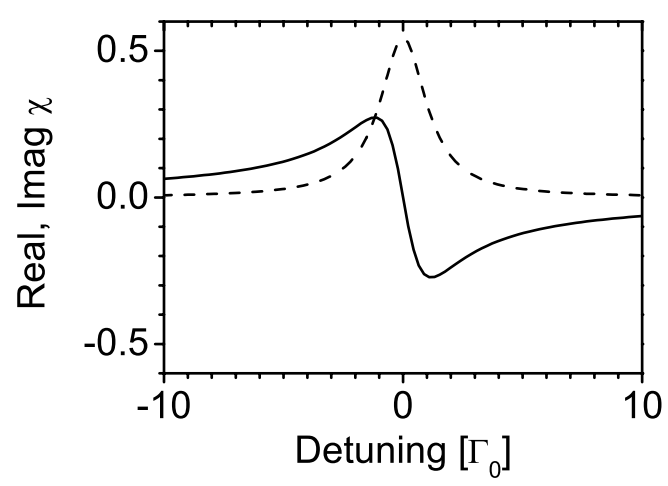

Figure 6.1.: Real (solid curve) and imaginary (dashed curve) Doppler-free electric susceptibility $(\chi=\epsilon-1)$ of $C$ s vapor at a density of $7.1 \cdot 10^{19} \mathrm{~m}^{-3}$.

unity above the resonant frequency $\omega_{0}$, see figure 6.1. To achieve $\epsilon^{\prime}(\omega)<1$, the transitions must be isolate. The isolation is necessary as otherwise $\epsilon^{\prime}(\omega)$ below resonance of the higher energy transition offsets the low $\epsilon^{\prime}(\omega)$ above resonance of the lower energy transition. To have $\epsilon^{\prime}(\omega)$ change considerably over resonance, it is desirable for the atomic medium to be dense. Since the imaginary part of $\epsilon(\omega)$, associated to absorption, is related to the dispersion by the Kramers-Kronig relations [67], $\epsilon^{\prime}(\omega)$ can differ considerably from unity while $\epsilon^{\prime \prime}(\omega)<<1$ for a select frequency range outside resonance, see figure 6.1. Thus, atomic systems have a large modulation of $\epsilon(\omega)$ which warrants a study of their effect on photonic systems.

Only a limited number of studies have been performed of infiltrating photonic crystals with resonant media. Infiltration of strongly absorbing dye in a photonic crystal was achieved in [169], and liquid crystals have also been proposed $[131 ; 172]$. The main disadvantage of dye is the low useful concentration, as Förster energy transfer [173], which is proportional to the density of dye, increases the homogeneous width [174]. The large inhomogeneous width of semiconductor quantum dots (e.g., $5 \%$ for CdSe [175]) at the desired densities precludes large changes in $\epsilon^{\prime}(\omega)$. To avoid inhomogeneous width by atoms, a system was proposed by van Coevorden [32]: here, the crystal consists of cold atoms in a lattice. A conceivable realization of this was obtained in $[176 ; 177]$, with a low lattice site occupancy of $<1 \%$. A bandgap is shown to open when the resonance wavelength equals the lattice spacing, for one atom per lattice site, a condition experimentally achieved recently [178], but where no photonic properties were probed. 


\subsection{2. ${ }^{133} \mathrm{Cs}$ - the ideal resonator}

In this section we will elaborate what medium is eligible to be infiltrated into a photonic crystal, and what its optical properties are. Consequently, we will discuss what the limitations of the maximum possible change in $\Delta \epsilon^{\prime}(\omega)\left(=\Delta \epsilon_{\max }^{\prime}\right)$ are, after showing how $\epsilon(\omega)$ can be derived from a simple model. We chose to use a hot, dense vapor of ${ }_{55}^{133} \mathrm{Cs}$ as a strongly polarizable resonant medium, Cs being the most suited of all alkali metals. As with all alkali metals, it has only one electron in the outer shell (state $6^{2} S_{1 / 2}$ ). For that reason, the transition from the ground state to the $6 P$ manifold (known as the $\mathrm{D}$ transition) is strong. In particular, we look at the $D_{1}$ transitions of ${ }^{133} \mathrm{Cs}$, which consist of four isolated hyperfine hyperfine transitions (see fig. 6.2(a)). The long lifetime of the excited state gives rise to a narrow intrinsic linewidth $\left(\Gamma_{0}=2 \pi 4.56 \mathrm{MHz}\right)$, and thus large polarizability at resonance [179-181]. In the ground state, the hyperfine splitting is $9.193 \mathrm{GHz}$, a wellknown transition which defines the unit of time [182].

The polarizability $\alpha(\omega)$ of a single atom at rest is given by (see, e.g., $[183 ; 184])$

$$
\alpha(\omega)=\frac{e^{2} f}{m_{e}} \frac{1}{\omega_{0}^{2}-\omega^{2}-i\left(\omega^{3} / \omega_{0}^{2}\right) \Gamma_{0}},
$$

where $\Gamma_{0}$ is the inverse lifetime of the excited state, $e$ and $m_{e}$ the charge and mass of the electron, and $\omega_{0}$ and $\omega$ the resonance and frequency in angular units, respectively. The absorption oscillator strength $f$ relates the strength of the Lorentz oscillator to a real electron in an atom subject to finite transition probabilities. For small detunings, $\Delta \equiv \omega-\omega_{0}$, and

$$
\alpha(\omega) \approx \frac{e^{2} f}{2 m_{e} \omega_{0}\left(\omega_{0}-\omega-i \Gamma_{0} / 2\right)},
$$

where we have made use of the rotating wave approximation. An ensemble of atoms at density $N$ at rest has the relative dielectric function $\epsilon(\omega)$ in mks units

$$
\begin{aligned}
\epsilon(\omega) & =1+\chi \\
& =1+N f \alpha(\omega) / \epsilon_{0} \\
& =1+\frac{f \omega_{P}^{2}}{2 \omega_{0}} \frac{1}{\left(\omega_{0}-\omega\right)-i \Gamma / 2} \\
& =1+\frac{f \omega_{P}^{2}}{2 \omega_{0}}\left(\frac{-\Delta}{\Delta^{2}+(\Gamma / 2)^{2}}+i \frac{\Gamma / 2}{\Delta^{2}+(\Gamma / 2)^{2}}\right),
\end{aligned}
$$


where $\Gamma=\Gamma_{0}+\Gamma_{b}, \Gamma_{b}$ the width to due elastic and inelastic collisions, $\omega_{P}^{2}=N e^{2} / \epsilon_{0} m_{e}$ the square of the plasma frequency, and $\epsilon_{0}$ the permittivity of vacuum. The maxima $\epsilon_{\max }^{\prime}$ and minima $\epsilon_{\min }^{\prime}$ are at $-\Gamma / 2$ and $+\Gamma / 2$, respectively. There can thus be a large change in $\epsilon^{\prime}(\omega)$ in just one linewidth. In contrast, the width of a $\mathrm{SiO}_{2}$ photonic crystal stopgap at $\nu=11178 \mathrm{~cm}^{-1}$ is around $612 \mathrm{~cm}^{-1}$, or $18.3 \cdot 10^{6} \mathrm{MHz}$, around 6 orders of magnitude larger than the intrinsic linewidth of the Cs hyperfine $D$ transitions.

At low temperatures $\left(T<250^{\circ} \mathrm{C}\right)$, Doppler broadening limits the change in $\epsilon^{\prime}(\omega)$ : an atom with a velocity $\mathbf{v}$ becomes excited when the excitation frequency equals $\omega=\omega_{0}+\mathbf{k} \cdot \mathbf{v}$, where $\omega_{0}$ is the resonance at rest and $\mathbf{k}$ the wavevector of the excitation beam. In any one dimension, the distribution of velocities is Gaussian. The FWHM width $\Delta \omega_{D}$ of this distribution is equal to $[185]$

$$
\Delta \omega_{D}=\frac{2 \omega_{0}}{c} \sqrt{\frac{2 R T \ln 2}{M}}
$$

where $M$ is the molar mass and $R$ the universal gas constant. The inhomogeneous Doppler width is taken into account by convolving $\chi(\omega)$ with the Doppler velocity distribution. At ambient temperature, and at the $D$ transitions, the lineshape of Cs vapor is determined by Doppler broadening.

As an example for both Doppler as well as Doppler-free spectra [186], we show transmission spectra of a monochromatic beam at frequencies around the $D_{1}$ line measured in a double-pass configuration through a $8 \mathrm{~cm}$ long Cs ampule (figure 6.2(b)). The four troughs correspond to the four hyperfine $D_{1}$ levels. Zero detuning is chosen at a frequency $20 \mathrm{GHz}$ below the lowest energy transition $\left(4 \rightarrow 3^{\prime}\right)$. Outside resonance, in the first pass, atoms of a particular velocity class are excited and saturated, but the reflected beam probes atoms of a different velocity class. Outside resonance thus the double-pass spectrum will look similar to an unsaturated transmission spectrum. At resonance, only those atoms are saturated for which $\mathbf{k} \cdot \mathbf{v}=0$ holds. Then, the reflected beam is not absorbed, because the reflected beam probes atoms of a same velocity class $\mathbf{k} \cdot \mathbf{v}=0$. Therefore, the centers of all four transitions show maxima, as shown in the inset. While the Doppler width of all four transitions is around $397 \pm 20 \mathrm{MHz}$, agreeing reasonably well with the calculated value of $361 \mathrm{MHz}$, the saturation peak at resonance has a width of $26 \mathrm{MHz}$, significantly higher than the expected $4.56 \mathrm{MHz}$. The deviation stems from a combination of possible power broadening and collision broadening.

We conclude that the large vapor pressure of $\mathrm{Cs}$, its narrow intrinsic linewidth, and its low sensitivity to Doppler broadening with temperature in 
(a)

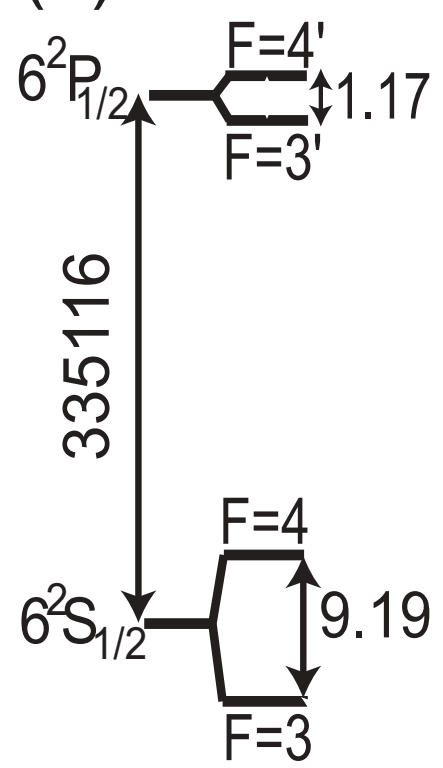

(b)

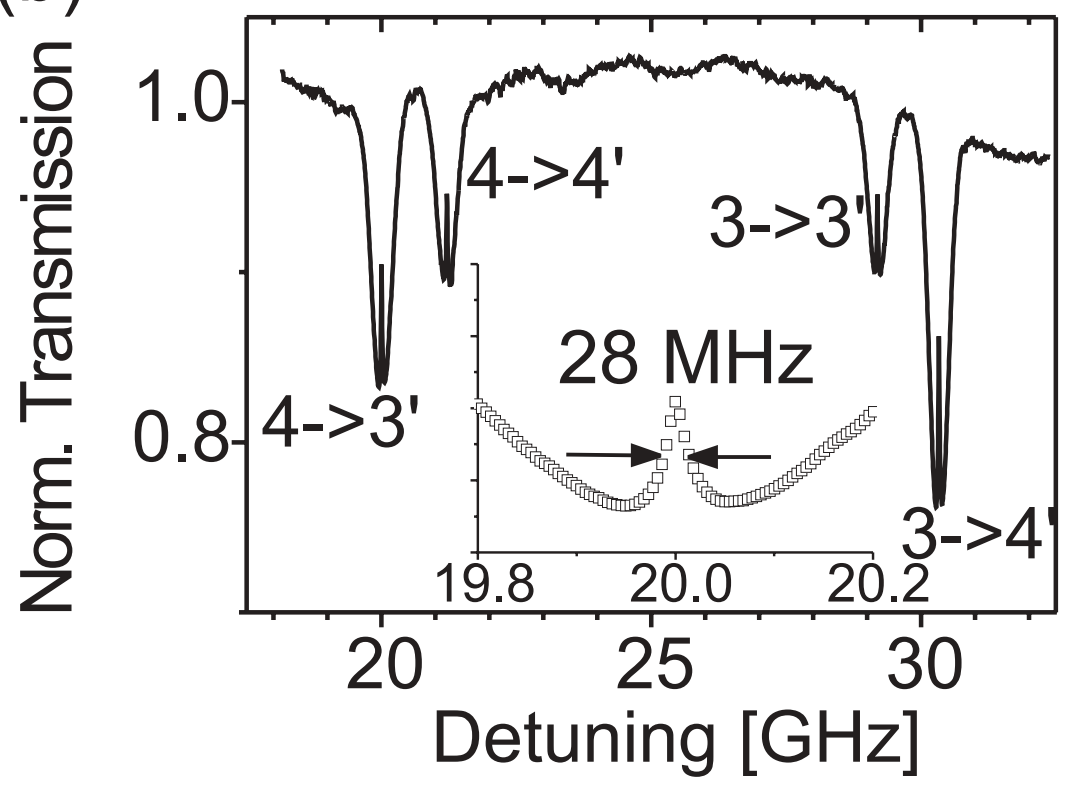

Figure 6.2.: (a) Grotrian diagram of the hyperfine $D_{1}$ transitions of Cs [187]. All units in GHz. (b) Double-pass transmission through an ampule of ${ }^{133} \mathrm{Cs}$ at room temperature. The 4 troughs correspond to the 4 Doppler broadened hyperfine $D_{1}$ transitions. The peaks in the centers of the absorption troughs are the Doppler-free resonances. Zero detuning is chosen at a frequency $20 \mathrm{GHz}$ below the lowest energy transition $\left(4 \rightarrow 3^{\prime}\right)$.

the temperature range of interest make it an ideal candidate for modifying a photonic crystal's optical properties.

\subsubsection{Cs as a strongly photonic medium}

In the following section, we discuss effects of collision broadening, which become dominant at elevated temperatures and will be shown to ultimately limit $\Delta \epsilon_{\max }^{\prime}$. The magnitude of the change in $\epsilon^{\prime}(\omega)$ in the Doppler-broadened regime depends on the density: the highest vapor density is reached near a liquid-vapor phase, i.e., above the melting temperature $\left(T_{M}=28.44^{\circ} \mathrm{C}\right)$. The vapor pressure in equilibrium is given by the Clausius-Clapeyron equation [188], and can be shown to vary as $\exp \left(-L_{0} / R T\right)$, where $L_{0}$ is the latent heat of vaporization and $R$ is the universal gas constant. Since the vapor pressure increases exponentially with temperature, large changes in density can be obtained by comparatively small changes in $T$, which seems opportune for large changes in $\epsilon^{\prime}(\omega)$. 
Chapter 6. Enhancement and reduction of an opal's photonic strength by atomic dispersion

\begin{tabular}{||l|l|l|l|l|l||}
\hline$T$ & $N$ & $\omega_{D} / 2 \pi$ & $\Gamma / 2 \pi$ & $\ell_{\text {abs }}$ at HM & $\Delta \epsilon^{\prime}$ \\
{$[K]$} & {$\left[\lambda_{0}^{-3}\right]$} & {$[\mathrm{MHz}]$} & {$[\mathrm{MHz}]$} & {$[\mu \mathrm{m}]$} & \\
\hline 300 & 0.0434 & 360.37 & 4.57 & 109350 & $2.44 \cdot 10^{-5}$ \\
\hline 370 & 9.20 & 400.21 & 5.68 & 577.12 & $4.71 \cdot 10^{-3}$ \\
\hline 400 & 50.85 & 416.11 & 10.77 & 110.31 & $2.47 \cdot 10^{-2}$ \\
\hline 420 & 138.38 & 426.39 & 21.46 & 42.91 & $6.42 \cdot 10^{-2}$ \\
\hline 500 & 3344.90 & 465.23 & 413.05 & 4.41 & 0.70 \\
\hline
\end{tabular}

Table 6.1.: This table shows optical parameters of interest of Cs vapor vs. temperature $T$. The density $N$ has been calculated from a Langmuir-Taylor expansion of the saturated Cs pressure [189], the Doppler width $\Delta \omega_{D} / 2 \pi$ from equation 6.7 and $\Gamma$ from [190] using a oscillator strength $f=0.3438$ [187]. The absorption length $\ell_{\text {abs }}$ at the half-maximum of the Doppler-broadened $\chi(\omega)$ has been extracted from the square root of a numerically generated dispersion curve from equation 6.6.

To compare, the saturated vapor pressure of $\mathrm{Rb}$ is 30-60 times lower, and its melting temperature is $39.31^{\circ} \mathrm{C}$ [189]. Moreover, $\mathrm{Rb}$ has two stable isotopes, ${ }^{85} \mathrm{Rb}$ and ${ }^{87} \mathrm{Rb},{ }^{1}$ which are not amenable to separation. Other alkali metals' vapor pressure is even lower, requiring prohibitively high temperatures to attain the desired density.

Outside the Doppler-broadening regime, at high temperatures, the width due to collision broadening exceeds that of Doppler broadening, see table 6.1. Here, an atom in the excited states interacts with a ground state atom. A phase variation in the resonant transition is incurred, which gives rise to new frequency components. Inelastic collisions transfer energy from the upper state to the lower state, and thus quench the upper state population. Since these mechanisms work on all atoms equally, it is a homogeneous broadening. The width due to collision broadening is proportional to the density $N$, and is described by a density-width constant of $N \Gamma_{b} / f=2 \pi \cdot 0.3$ $\mathrm{cm}^{-3} \mathrm{~s}^{-1}$ for the Cs $D_{1}[190]$.

We will now argue that the precise magnitude of Doppler broadening governs the temperature at which the change from Doppler- to collision broadened regime occurs. At the extrema of $\epsilon^{\prime}(\omega), \epsilon^{\prime}(\omega)=1 \pm f \omega_{P}^{2} / 4 \omega_{0}\left(\Gamma_{b}+\right.$ $\left.\Gamma_{0}\right)$. For atoms at rest, the obtainable strength asymptotically approaches a maximum as from $\Gamma_{0}=\Gamma_{b}$, which is at $N=5 \cdot 10^{19} \mathrm{~m}^{-3}$, giving $\Delta \epsilon^{\prime}=0.47$. In a bulk vapor whose Doppler width is given by equation 6.7 , that $\Delta \epsilon^{\prime}$ is not obtained until a much higher density of $N=2.7 \cdot 10^{21} \mathrm{~m}^{-3}$, corresponding to $T=200^{\circ} \mathrm{C} . \Delta \epsilon_{\max }^{\prime}$ can thus be reached by operating outside the Doppler-

\footnotetext{
${ }^{187} \mathrm{Rb}$ is not stable, but has a lifetime of a few billion years, and is thus effectively stable.
} 
broadened regime, i.e., by increasing the temperature and thus the vapor pressure. In principle thus, Doppler broadening does not limit $\Delta \epsilon_{\max }^{\prime}$. A precise estimation of Doppler broadening mechanisms in our situation is thus essential if the correct temperature is to be chosen. The following sections will show how Doppler broadening close to resonance can indeed be reduced close to an interface, after some basic properties of reflection spectra of vapors have been discussed.

\section{Reflection of a vapor behind a glass interface}

The reflectivity probed by a light beam passing from a dielectric with index $n_{G}$ into an atomic vapor of refractive index $n_{v}^{\prime}(\omega)+i n_{v}^{\prime \prime}(\omega)$ is given by [191]

$$
R(\omega)=\frac{(m(\omega)-1)^{2}+\kappa^{2}(\omega)^{2}}{(m(\omega)+1)^{2}+\kappa^{2}(\omega)},
$$

where $m(\omega)+i \kappa(\omega)=\left(n_{v}^{\prime}(\omega)+i n_{v}^{\prime \prime}(\omega)\right) / n_{G}$. For low gas densities, or far from resonance, $\kappa^{2}(\omega)$ can be neglected, and $m(\omega)$ can be expanded as $m_{0}+\delta m(\omega)$. The resulting reflectivity is

$$
R(\omega)=\frac{\left(m_{0}-1\right)^{2}}{\left(m_{0}+1\right)^{2}}+\frac{2 \delta m(\omega)\left(m_{0}-1\right)}{\left(m_{0}+1\right)^{2}},
$$

where we have also assumed $\left(m_{0}+1\right)>>2 \delta m(\omega)$. For the above conditions, the change in reflectivity of a vapor depends purely on $\delta m(\omega)$. At high densities however, especially at resonance, when $n_{v}^{\prime \prime}(\omega)$ is apreciable, $n_{v}^{\prime \prime}(\omega)$ has a considerable influence on $R(\omega)$ : as seen in eq. $6.6, n_{v}^{\prime \prime}(\omega) \approx 1+\frac{1}{2} \operatorname{Im}(\chi)$ decreases as the detuning squared, while $n_{v}^{\prime}(\omega) \approx 1+\frac{1}{2} \operatorname{Re}(\chi)$ decreases linearly with detuning. While the tail in the wings is still indicative of $n_{v}^{\prime}(\omega)$, the reflectivity close to resonance is modified strongly by $n_{v}^{\prime \prime}\left(\omega=\omega_{0}\right)$. The extrema in $R(\omega)$ are therefore not coincident with extrema in $n_{v}^{\prime}(\omega)$.

\section{Selective reflection}

An effect known as selective reflection $[192 ; 193]$ essentially destroys Doppler broadening at resonance. Atoms with a velocity component $v_{\|}$parallel to the monochromatic beam must have suffered a collision with the wall, which is most probably inelastic [194]. The phase of the transition has been scrambled, or the atoms have decayed to the ground state. In both cases they will not be amenable to polarization for a maximum of $1 / \Gamma_{0}=35 \mathrm{~ns}$ (for an atom on resonance). In that time it will have travelled around $10 \mu \mathrm{m}$, a sizeable 


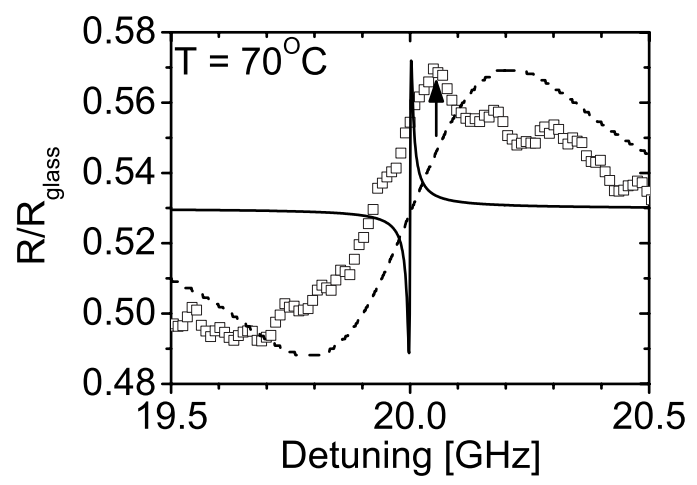

Figure 6.3.: Oblique $\left(27 \pm 2^{\circ}\right)$ reflection of the $4 \rightarrow 3^{\prime}$ transition of a moderatly hot Cs vapor $T=70^{\circ} \mathrm{C}$ versus frequency. A sharp peak can be observed at $21 \mathrm{GHz}$, indicated by the arrow. To compare, the Doppler-broadened (dashed curve) and unbroadened (solid curve) lineshapes are also plotted. The theoretical curves have been scaled and offset for clarity.

fraction of the absorption length of $\ell_{\mathrm{abs}}=55 \mu \mathrm{m}$ at $T=130^{\circ} \mathrm{C}$. Atoms with antiparallel velocity components also need time $35 \mathrm{~ns}$ to polarize with the probe field. Thus atoms with large $\pm v_{\|}$will hardly be probed, leading to a narrowing of the spectral line. As an approximation, only atoms for which $\ell_{\text {abs }}>\bar{v} / \Gamma$ holds will be probed.

As an example, we have probed the reflection of hot vapor Cs over the $D_{1}$ transitions. The vapor is confined behind a glass interface. The monochromatic, scannable laser beam is incident at an angle of $\theta=16 \pm 2^{\circ}$ (externally $27 \pm 2^{\circ}$ ). The effect of the oblique angle is that the Doppler width is rebroadened by $\Delta \omega_{D} \sin (\theta)$ [194]. The results are shown in figure 6.3. Just blue of the resonance $(21 \mathrm{GHz})$, we observe a sharp peak, while at larger blue detunings, the peak considerably broadens. The reflection spectrum for a collision broadened transition is calculated, with Doppler broadening (dashed curve) and Doppler-broadening (solid curve). While the Doppler broadened spectrum matches well at large detunings, close to resonance the measured spectrum is much sharper. We conclude that boundaries effectively decrease the width of the velocity distribution, although the effect of wall collisions has not been included in our analysis. However, since the self-broadening mean free path scales as $N^{-\frac{1}{3}}$, but the wall collision mean free path scales as $N^{-1}$, wall collisions become negligible for higher densities. The decrease of the effective velocity distribution may lower the temperature for which $\Delta \epsilon_{\max }^{\prime}$ is reached. 


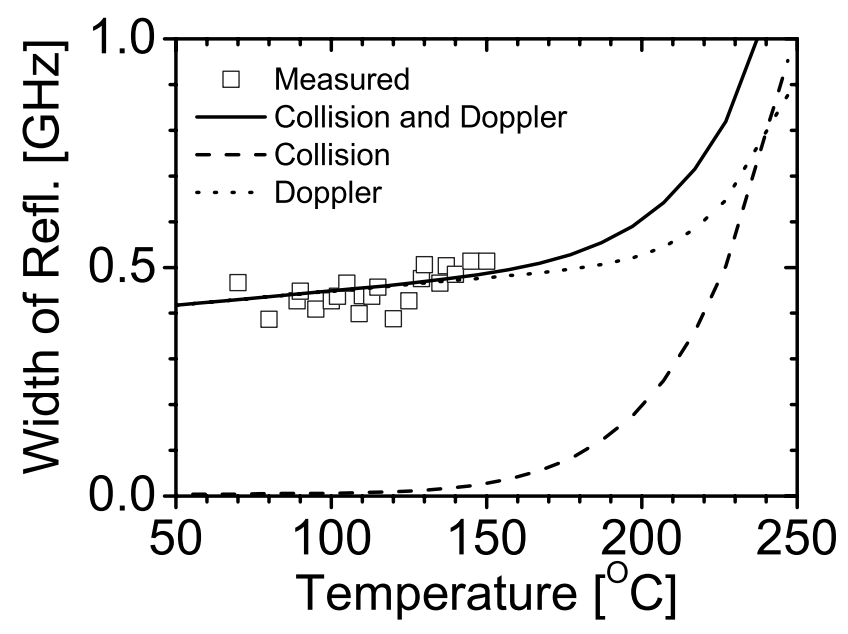

Figure 6.4.: Measured Width (FWHM) of the reflectance at $27^{\circ} \pm 2$ of the hyperfine $4 \rightarrow 3^{\prime} D_{1}$ transition of Cs vs. temperature at saturated vapor pressure. We calculate the linewidth from the Lorentz model, convolved with a Gaussian of a width corresponding to Doppler broadening (solid curve). The linewidth without such an inhomogeneous broadening is shown by the dashed curve. Some raw data is shown in figure 6.3.

\section{Reflection at large detunings}

In the last section we focussed on the narrowed spectral width close to resonance due to selective reflection. In this section, the properties of $R(\omega)$ far from resonance are discussed, for which selective reflection is negligible. For all temperatures, the widths as determined from the extrema of the reflectivity spectra (fig. 6.3) are plotted in figure 6.4. We observe a slow increase in width with temperature, in agreement with the square root increase expected from eq. 6.7 in the Doppler limited regime. To compare, the widths expected from an exclusively Doppler broadened (dotted curve) or collision broadened $R(\omega)$ have also been plotted. At low temperatures, the width is dominated by Doppler broadening. At $T=150^{\circ} \mathrm{C}$, the effects of collision broadening become significant, and at $250^{\circ}$, the width due to collisions has overtaken the widths due to Doppler broadening, as expected.

From the last sections we conclude that for $T<250^{\circ} \mathrm{C}$, the change in $\epsilon^{\prime}(\omega)$ over resonance is limited by Doppler broadening. Selective reflection reduces Doppler broadening near resonance of atoms bounded by an interface, and it has unknown consequences for a vapor in a photonic crystal. 


\subsection{4. $\mathrm{SiO}_{2}$ opals as photonic media}

Having discussed requirements of the polarizable medium, we now turn to the desirable properties of the photonic sample. The most important condition to be met is that the photonic media be chemically stable. While not anticipated, it is deemed possible that the chemically highly active vapor corrodes the samples at hand. If the sample's chemical inertness to Cs cannot be vouchsafed, the samples must be available inexpensively and quickly. For this reason, strongly photonic 2 or 3D photonic crystals fabricated by state-of-the-art processing techniques are not appropriate. 1D samples with air as the main dielectric are not available, although porous Si crystals have been proposed [195]. In contrast, thick and large photonic crystals made from self-assembled colloids can be grown within a few days [40-42] and can be inverted to achieve a higher air fraction [46]. Colloidal spheres are usually well characterized and of low polydispersity $\left(2-4 \%\right.$ for $\mathrm{SiO}_{2} \mathrm{mi}-$ crospheres). High quality opals can be grown from polystyrene, but the low glass transition temperature of $100^{\circ} \mathrm{C}$ [189] is incompatible with the high temperatures desired for a high $\mathrm{Cs}$ density. $\mathrm{TiO}_{2}$ inverse opals were found to corrode within a few hours of Cs contact. Because the experimental cell in which the vapor was probed contained $\mathrm{SiO}_{2}$ which appeared not to be attacked for several days, we opted to use $\mathrm{SiO}_{2}$ opals, layers grown on the window substrate.

Optically, the most important condition is that the blue edge of a stopgap of the photonic crystal be at the Cs $D_{1}$ resonance. Then, the change in the vapor's dielectric function will have large effects on the change in reflectivity $R(\omega)$, as the gradient of the reflectivity is highest at the edges of the stopband. While the antinodes of the probe's electric field are preferentially in the dielectric for probe frequencies near the red edge of the stopgap, high electric fields are in the voids of an opal at the blue edge [8]. Therefore, $\mathrm{SiO}_{2}$ opals are desired whose stopgap is slightly red shifted with respect to the Cs $D_{1}$ resonance.

\subsection{Experimental setup}

\subsubsection{Excitation of ${ }^{133} \mathrm{Cs}$}

The resonances are probed by exciting the hyperfine transitions with ultra narrowband single mode cw laser (MBR-110, Coherent), pumped by a frequency doubled Nd:YAG (Verdi V-10). Once locked, the laser can scan over 


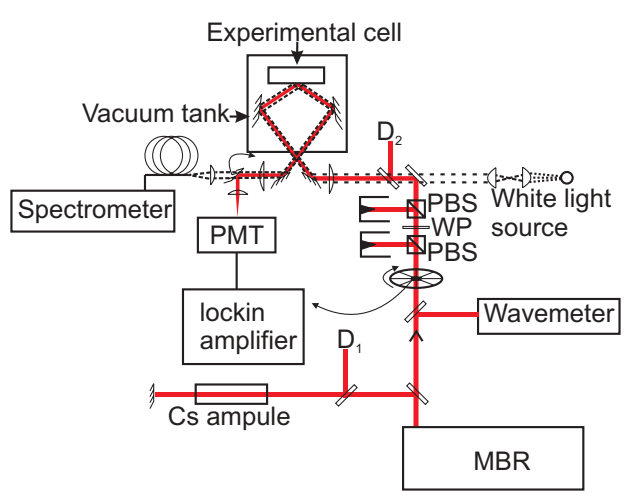

Figure 6.5.: Figure of experimental setup. The $1 \mathrm{~W}$ output of the linearly polarized light from the narrowband tunable laser (MBR) gets attenuated by polarizing beamsplitters $(P B S)$ and a $\lambda / 2$ waveplate $W P$ after being modulated by a chopper. The diode $D_{2}$ monitors the power. The reflected light from the sample in the heated experimental cell is picked up by the photomultiplier tube (PMT). For a fine frequency reference, diode $D_{1}$ measures the saturated absorption from a reference ampule (see figure 6.2), while a coarse reference is performed by the wavemeter. Light from a halogen lamp is used to measure the optical properties of the sample in the experimental cell (for details of experimental cell, see fig. 6.6.). A vacuum pump evacuates the tank to reduce air convection around all heated elements.

$40 \mathrm{GHz}$ and can thus excite all four transitions in one scan, and probe the dispersion region. The $1 \mathrm{~W}$ linearly polarized beam is attenuated by two polarizing beamsplitters and a $\lambda / 2$ waveplate combination (Lens Optics, Germany) after being amplitude modulated by a chopper (see figure 6.5). The beam is focussed by an achromat $(\mathrm{f}=300 \mathrm{~mm})$ under an angle of $\theta=27 \pm 2^{\circ}$ onto the experimental cell containing the vapor and the opal. The reflection of the narrowband source is collected by a Hamamatsu R928 Photomultiplier tube (PMT). Note that the extremely small solid angle subtended by the PMT at the viewport $\left(\Omega \approx 10^{-6} \mathrm{sr}\right)$ essentially prevents fluorescence being measured. The PMT's photocurrent was measured by a phase sensitive amplifier, where the time constant was chosen to be $\tau=30 \mathrm{~ms}$, or one sample period. While the coarse laser frequency was monitored by a Burleigh WA-10L wavemeter (resolution: $0.1 \mathrm{~cm}^{-1}$ ), the precise frequency was measured with Doppler free spectroscopy (see figure 6.2). To verify the photonic structure stayed intact, a much wider bandwidth is required: broadband reflectivity was measured with a spatially-filtered halogen white light source and a VIS-NIR 2048 channel spectrometer (Ocean Optics, USB 2000). 


\subsubsection{Control of the vapor}

The experimental setup is depicted in figure 6.6. The need for a high vapor density required heating liquid Cs contained in the reservoir. Therefore, a cell was designed in which Cs can be both be heated as well as spatially confined. Heating was accomplished by two heating elements (Thermocoax) brazed into the cylindrical reservoir at the top and the bottom. Upon opening valve $V_{1}$ (Swagelok SS-4BG-V51), hot Cs vapor diffuses into the experimental cell, also heated by two heating elements. Because Cs reacts strongly with both oxygen and water, the reservoir and experimental cell are kept under vacuum $(<10 \mu$ bar, detection limited by the low conductance $(0.08 \mathrm{l} / \mathrm{s})$ of the valve and the thin tube [196]). To discard any moisture and oxygen, the assembly was baked and evacuated at $150^{\circ} \mathrm{C}$ by a Turbo-Molecular pump (Pfeiffer Balzers TPH-170, pump speed $170 \mathrm{l} / \mathrm{s}$ ) and a rotary valve pump DUO-008 B (2 l/s) for at least 24 before admitting Cs. The assembly is evacuated through valve $V_{2}$. The heating to the desired temperature would cause air convection around the experimental cell, were it not for the vacuum tank of volume $=0.037 \mathrm{~m}^{-3}$ in which the optical assembly consisting of reservoir, valves, and experimental cell is mounted. Therefore, the tank was kept under vacuum $\left(10^{-3}\right.$ mbar $)$ throughout. A second advantage of the tank was that hazards due to possible leaks in the assembly are contained. Also, the reduced pressure difference between the evacuated tank and the experimental cell would reduce the flow rate into the experimental cell because of a possible leak. The experimental cell was leak tested with a Balzers HLT-160 before every experimental run to verify that the leak rate was $<10^{-9} \mathrm{mbar} 1 / \mathrm{s}$. A disadvantage of the tank was that once the experiment was running, no adjustments could be made. At the end of each experiment, a thin diffuse film was observed on the reflecting optics, degrading the reflectivity. Both the reservoir and the experimental cell were independently temperature controlled by a 2604 PID temperature controller. The temperature was measured by ring J-type thermocouples. Heatingtape was wound around the tubes and valves to prevent cold spots, which would otherwise determine the vapor pressure of Cs. The experimental cell, valves and tubes are kept at a temperature $30-50 \mathrm{~K}$ above that of the reservoir to prevent condensation. In independently performed calibration runs we found the time-constant of the thermal system to be $1 \mathrm{~h}$.

The experimental cell required particular attention, as it comes in contact with both well characterized samples and aggressive Cs vapor [197]. After several experimental runs we found that viewports by Hositrad, The 


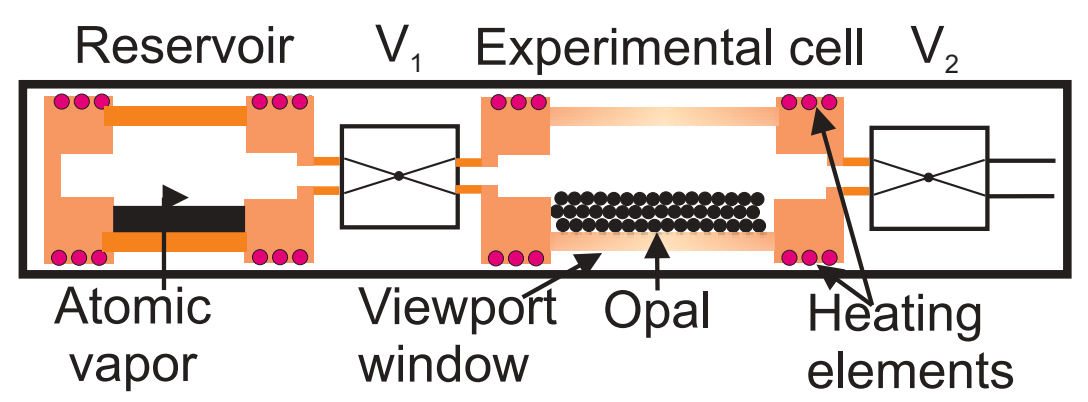

Figure 6.6.: The optical assembly including the experimental cell, two valves, and the reservoir. The experimental cell is connected via valve $V_{1}$ to a reservoir containing liquid $C$ s. At the other side valve $V_{2}$ closes the experimental cell off from a vacuum pump.

Netherlands, showed substantial cracks and leaks after having been in contact with Cs for only a few hours, which we attributed to corrosion of the Kovar metal-borosilicate glass seal. Viewports by VacGen, England showed no such degradation over several days. Moreover, these viewports protruded less into the tank, which enabled probing at a much larger angle. Oblique probing was necessary for two reasons: first, it largely eschewed the $2 \mathrm{~mm}$ thick window acting as a Fabry-Pérot étalon, with a fringe spacing of $1 /(2 \cdot 0.2$ $\left.\mathrm{cm} \cdot n_{\text {glass }}\right)=1.7 \mathrm{~cm}^{-1}$, or $50 \mathrm{GHz}$, just over the scan range of the laser. Second, it blue shifted the reflectivity peaks of the opal to the Cs resonance, using the angle as tuning parameter.

\subsubsection{Samples}

\section{Initial experiments}

In initial experiments, $\mathrm{TiO}_{2}$ inverse opals were used as samples [46]. $\mathrm{TiO}_{2}$ has a large refractive index of 2.5 at $\lambda=1 \mu \mathrm{m}$, giving rise to a high photonic strength. Moreover, inverse opals have a low filling fraction which permits a high density of $\mathrm{Cs}$, around 3 times higher than that in opals. $\mathrm{TiO}_{2}$ inverse opals can be grown with a thickness of several hundreds of lattice spacings, giving rise to high reflectivities and they are thus sensitive to changes in the vapor's refractive index. In contact with Cs however, the inverse opals degraded rapidly and were rendered unrecognizable. Moreover, the surface roughness, estimated to be in the order of a several sphere diameters $(\Phi=$ $520 \mathrm{~nm})$, permitted a finite thickness of vapor between the glass and the opal, and caused the beam to be largely absorbed at resonance $\left(\ell_{\mathrm{abs}}(T=\right.$ $\left.150^{\circ} \mathrm{C}\right)=21 \mu \mathrm{m}$ ), thus not probing the inverse opal. 


\section{Sample preparation}

To prevent surface roughness and an absorbing vapor under the sample, $\mathrm{SiO}_{2}$ opals were grown on the viewport windows themselves. A vial and viewports are cleaned overnight in a solution of $30 \mathrm{~g} \mathrm{NaOH}, 30 \mathrm{ml}$ of ultra-pure water, and $200 \mathrm{ml}$ of ethanol. All glassware is then rinsed first in water from a Direct-Q system and then ethanol. The glassware is thoroughly dried in a stream of nitrogen. A suspension of $0.1-0.5 \% \mathrm{SiO}_{2}$ colloids in ethanol is placed in the dried vial. The viewport is inserted at an angle between $40^{\circ}$ and $60^{\circ}$ into the vial, which is heated in an oven to between $30^{\circ} \mathrm{C}$ and $60^{\circ} \mathrm{C}$. Upon evaporation of the suspension, the colloids move towards the area of fastest evaporation, which is the wetting (suspension) film, and get deposited on the substrate. The structure of one such array is close packed. As the evaporation proceeds, more and more of these arrays form on top of each other until finally there is no suspension left and the growth stops [40]. Despite consistently scanning the parameter space in temperature, angle, and colloid density, the opals on the viewports were of low quality, with a reflectivity of $2 R_{\text {glass }}$, where $R_{\text {glass }}$ is the reflectivity of viewport window. Opals grown on microscope slides showed clearly defined peaks in excess of $13 R_{\text {glass }}$. From optical microscope images, the layer thickness was estimated to be between 1 and 3 (111) lattice spacings. However, since greenish opalescence was observed, we use the term opal for these thin structures.

Figure 6.7(a) shows the reflectivity spectrum of such an opal. In this chapter only, reflectivity is measured with respect to $R_{\text {glass }}$ of the viewport window. Two broad peaks are observed at $10800 \mathrm{~cm}^{-1}$ and $18500 \mathrm{~cm}^{-1}$, while the latter seems to be split into 3 separate peaks. While the origin of the triple peak is not well understood, it has been observed elsewhere [57]. The large width of $48 \%$ is due to lack of periodicity in the beam direction. At $11178 \mathrm{~cm}^{-1}$, the Cs $D_{1}$ resonance has been indicated (dashed line), which is just blue of the first peak, as aimed for. The absence of FabryPérot fringes indicates that the fringes are averaged out due to disorder. The bandstructure from $\mathrm{L}$ to $\mathrm{U}$ for a $\mathrm{SiO}_{2}$ opal ( $\mathrm{n}^{\prime}=1.45$ ) has also been plotted (b). The peak at $10800 \mathrm{~cm}^{-1}$ corresponds well to the frequency expected from the forbidden band. Only one of the small peaks within the broad peak at $18500 \mathrm{~cm}^{-1}$ corresponds well to the forbidden band. The second peak is probably due to the presence of higher order gaps and bands to which external light cannot couple. 


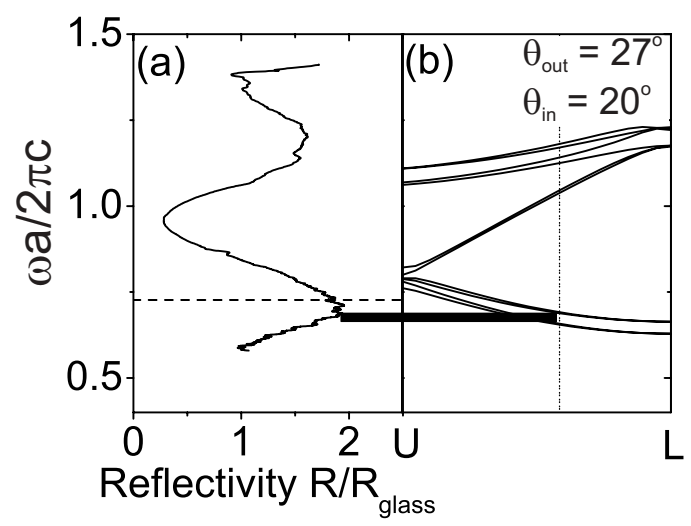

Figure 6.7.: (a) Reflectivity spectrum of the $\mathrm{SiO}_{2}$ opal grown on the viewport with respect to the reflectance of the viewport, probed at oblique incidence $\left(27 \pm 2^{\circ}\right.$, internally $\left.20 \pm 2^{\circ}\right)$. The first peak at $10050 \mathrm{~cm}^{-1}$ can be identified with the first order stopgap of a $\mathrm{SiO}_{2}$ opal. The second reflectivity peak is probably due to flat bands and higher order stopgaps to which external light cannot couple to. The vertical dashed line indicates the resonance of the Cs $D_{1}$ transition. (b) Bandstructure from $X$ to $U$ in the irreducible part of the Brillouin zone of an fcc crystal. The black bar indicates the forbidden band at an internal angle of $20 \pm 2^{\circ}$.

\subsection{Results and Discussion}

\subsubsection{Reflection at Cs resonances}

In a typical measurement, spectra of the resonances were taken while sweeping the laser frequency while the Cs density was controled by changing the reservoir temperature. After evacuating both the vacuum tank and subsequently the optical assembly, the temperature of the reservoir was increased to $100^{\circ} \mathrm{C}$ after which it was increased by $10-20^{\circ} \mathrm{C}$ every hour. At the beginning and at the end of every such ramp, a broadband reflection spectrum was taken.

Figure 6.8(a) to (d) show the narrowband spectra at the end of each ramp. The four hyperfine resonances can clearly be observed. Panel (a) shows a spectrum for $T=100^{\circ} \mathrm{C}$. Its base reflectivity is around $0.065 R_{\text {glass }}$, or $0.26 \%$. At the $4 \rightarrow 3^{\prime}$ transition, the measured spectrum shows a short tail with a sizeable trough. Close to resonance, the reflectivity peaks strongly. With increasing temperature (b), the peak becomes more pronounced, in contrast to the trough, but its tail gets longer. Note also that the base reflectivity has decreased to 0.013 . At $140^{\circ} \mathrm{C}$, only $10 \mathrm{~K}$ higher, the spectrum has changed 
Chapter 6. Enhancement and reduction of an opal's photonic strength by atomic dispersion

Figure 6.8: Measured narrowband reflection spectra of the Cs hyperfine $D_{1}$ transitions. The exact resonances are marked by the dashed lines. From panel (a) to (c), the Cs vapor density has increased by increasing the temperature $T$. These narrowband spectra correspond to the broadband spectra shown in fig. 6.9.

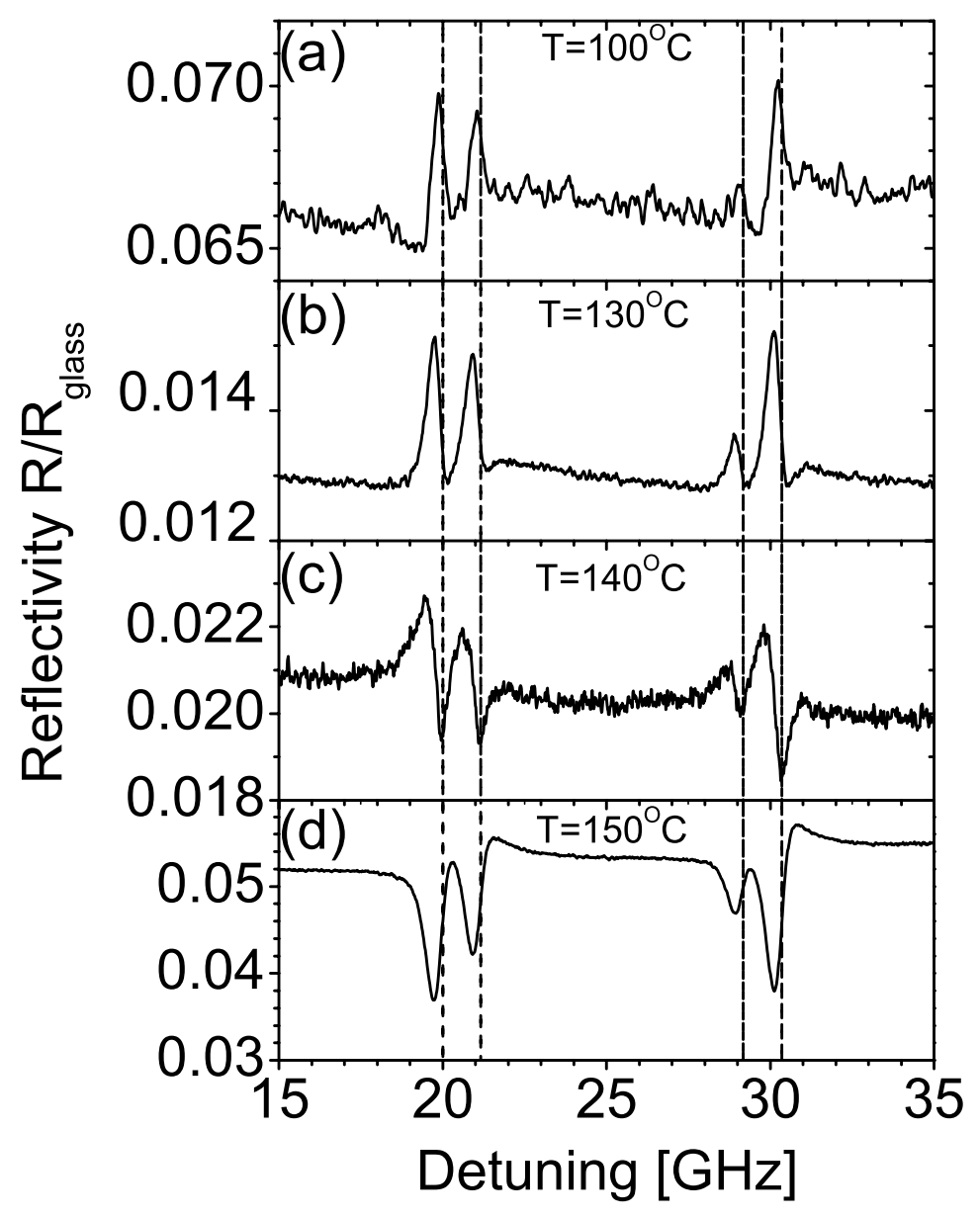

dramatically (c): a trough after the first peak has appeared, the trough being much below the baseline. The initial trough below resonance has completely disappeared. The baseline has recovered to 0.02 . In (d) $\left(T=150^{\circ} C\right)$, yet another $10 \mathrm{~K}$ higher, another striking change occurs: the peak as seen in (c) and (b) has now vanished and has completely given way to a marked trough. The complete spectra is without any peaks, exactly the opposite to (b). Moreover, the baseline has recovered even further to 0.05. We will discuss the spectra after inspection of the broadband data.

\subsubsection{Reflection of the opal}

To understand why the lineshape, baseline and strength of the reflectivity change with vapor temperature it is instructive to inspect the broadband reflectivity spectra, see left set of panels in figure 6.9. At first contact with Cs, even before spectra are observed, the first peak has shifted from 10800 
$\mathrm{cm}^{-1}$ to $9770 \mathrm{~cm}^{-1}(9.5 \%)$, while the second peak has shifted from 18500 $\mathrm{cm}^{-1}$ to $16300 \mathrm{~cm}^{-1}(12 \%)$. With increasing vapor temperature, the first peak shifts out of the spectrometer's spectral range, but second peak can still be traced. At a vapor temperature of $T=130^{\circ} C$, the second reflectivity peak has shifted to $14900 \mathrm{~cm}^{-1}$, or $20 \%$. At $T>140^{\circ} \mathrm{C}$, the peaks cannot be identified unambiguously. This is because the overall reflectivity decreases with increasing temperature, and is attributed to the thin oily film which settled on the reflecting optics in the tank during the experiment.

The shifting of the reflectivity peaks is attributed to the partial reduction of the $\mathrm{SiO}_{2}$ colloids [198]. Since Cs is strongly caustic, it will reduce $\mathrm{SiO}_{2}$ to $\mathrm{SiO}_{x}$ [199], where $x$ varies from 2 to 0 , and its refractive index varies between that of $\mathrm{SiO}_{2}\left(\mathrm{n}^{\prime}=1.45\right)$ and that of $\mathrm{Si}\left(\mathrm{n}^{\prime}=3.5\right)$ [200]. The peak is thus expected to red shift according to Bragg's law containing the average refractive index (see eq. 1.1). If an outer shell of the colloids has completely been reduced to $\mathrm{Si}$, we estimate the reduced volume to be $18 \%$, or a shell thickness to be $9 \mathrm{~nm}$. If a homogeneous reduction to $\mathrm{SiO}$ has taken place, the colloids' refractive index matches that of $\mathrm{SiO}$ well $\left(n_{\mathrm{SiO}}=2.0 \pm 0.1\right)$. In practice, there will be a mixture between $\mathrm{SiO}_{x}$ and $\mathrm{Si}$ present in the colloids.

To compare to theory, a Transfer-Matrix calculation was performed on a monolayer of colloids surrounded by an atomic vapor, and probed at an angle of $27^{\circ}$ (see Appendix A). In the right hand side of figure 6.9, a Transfer Matrix calculation of a single layer of colloids is shown, where the probe beam is incident at a similar angle in the experiment. Here, the colloids' refractive index is changed as indicated, and is chosen to match the shifting second peak on the left. For the reference spectrum, before contact with $\mathrm{Cs}$, the agreement is excellent (first row). Both the absolute reflectivity and the spectral positions of the two peaks match well, although the calculated second peak is at a higher frequency than that of the measured peak. This is because the Transfer Matrix calculation cannot take into account the bandstructure, whose bands give rise to the second peak. The $D_{1}$ resonance is just blue of the first peak. On first contact with Cs, both the first and second peak red shift (not shown). At $T=100^{\circ} \mathrm{C}$, the second peak has shifted to $9770 \mathrm{~cm}^{-1}$, which is matched by the calculation for $n^{\prime}=1.95$. The $D_{1}$ resonance is at the blue foot of the first order peak. At $T=130^{\circ} \mathrm{C}$, the $D_{1}$ resonance is exactly in the trough between two peaks, obtained for $n^{\prime}=2.0$. As the temperature is increased even further, the second peak shifts so that the $D_{1}$ resonance is to the red of the second peak, for which an $n^{\prime}=2.15$ is needed.

Using the information obtained from the broadband spectra, we will dis- 


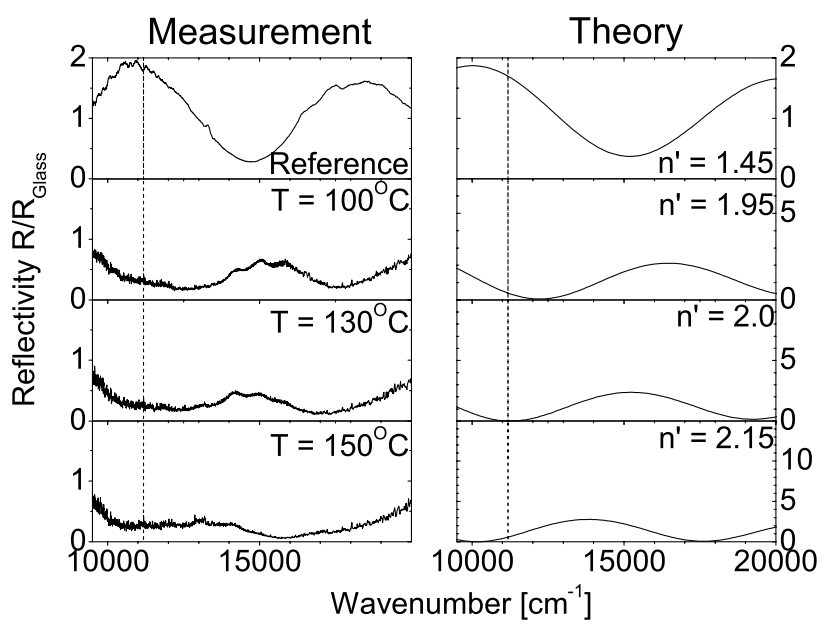

Figure 6.9.: Left side: Measured broadband reflectivity of the sample at different temperatures. The second reflectivity peak red shifts with increasing temperature. On first contact with of the opal with Cs, the peaks already red shift (not shown). The $D_{1}$ transition is marked by the vertical dashed line. Right side: Transfer-Matrix calculation of a layer of $\mathrm{SiO}_{x}$ colloids, where $x$ decreases from 2 to 0, resulting in an increasing effective refractive index $n^{\prime}$.

cuss the effects of a polarizable medium inside a photonic structure, whose reflectivity peak shifts due to a changing refractive index. We identify 4 characteristic cases:

1. An atomic resonance at the center of the reflectivity peak will lead to following behavior, as detected by a reflected beam. Since the gradient of the reflectivity peak at its maximum is small, the induced shift by the change in real $n_{v}^{\prime}$ will have only little effect on measured reflectivity. Also, because $\Delta n_{v}^{\prime}$ is small, the effect on the photonic strength will be marginal. The imaginary part of $n_{v}$ however will cause remove the constructive interference, and thus an increase in $n_{v}^{\prime \prime}$ will cause a trough at resonance.

2. When the resonance of the vapor is at the blue edge of the reflectivity peak, an increase in $n_{v}^{\prime}$ from unity (below resonance) will cause the stopgap to red shift for that frequency. A red shift at the blue edge of a peak lowers reflectivity. Above resonance, the opposite happens, as $n_{v}^{\prime}$ is below unity. The change in $n_{v}^{\prime}$ over resonance thus results in an anticorrelated reflection. At resonance, $n_{v}^{\prime \prime}$ will increase absorption, 


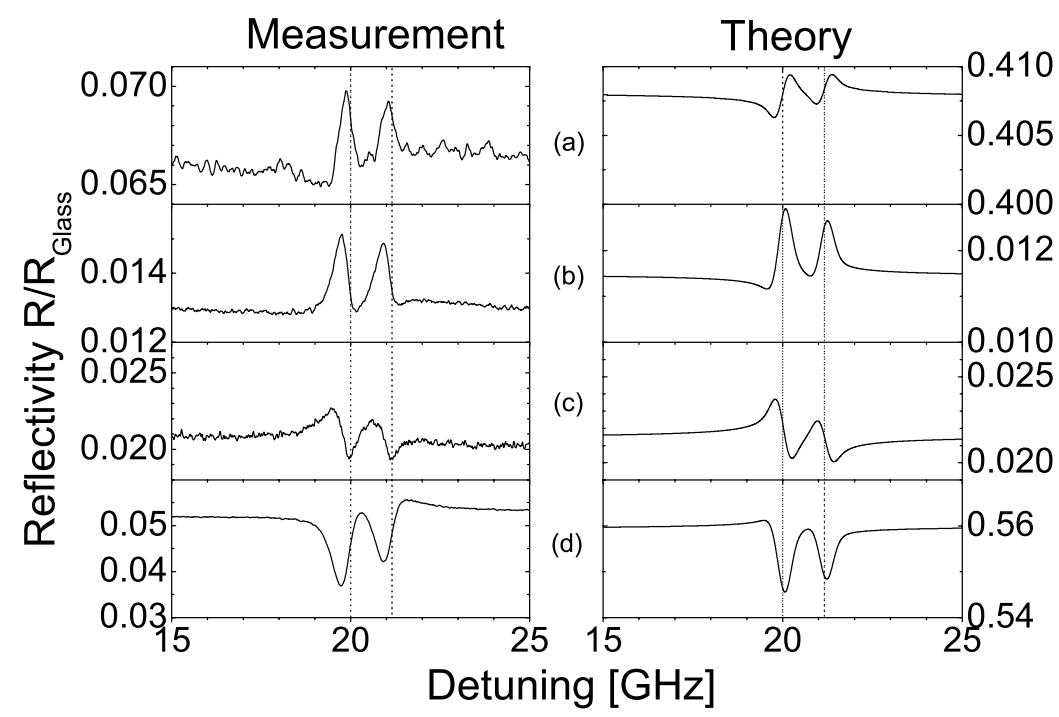

Figure 6.10.: Left set of panels: Measured narrowband reflection spectra of the $4 \rightarrow 3^{\prime}$ and $4 \rightarrow 4^{\prime}$ transitions (from fig. 6.8). The exact resonances are marked by the dashed lines. Right: spectra calculated with an extended Transfer-Matrix model including the absorption and dispersion of Cs.

and thus destroy the destructive interference, thereby increasing the reflectivity.

3. For a resonance exactly at the trough between the two reflectivity peaks, only the change in $n_{v}^{\prime \prime}$ will have an effect on the reflectivity, just as in the $1^{\text {st }}$ case. The gradient of the opal's reflectivity vanishes, and any change in $n_{v}^{\prime}$ will, while shifting the reflectivity peaks, result in no change in $R(\omega)$. The increase in $n_{v}^{\prime \prime}$ will destroy destructive interference, thus showing a peak at resonance.

4. At the red edge of the second peak, the increase in $n_{v}^{\prime}$ below resonance will red shift the peak, with the result this time that the reflectivity rises, while the $R(\omega)$ decreases for $n_{v}^{\prime}<1$ above resonance. The measured reflectivity has positive correlation with $n_{v}^{\prime}$. The effect of increase in $n_{v}^{\prime \prime}$ depends on whether the resonance is at the foot of the opal reflectivity peak or close to the summit: in the former case, destructive interference is removed, while in the latter case, constructive interference is removed.

In figure 6.10, right hand side, we show the same Transfer-Matrix calcu- 
lations as in figure 6.9, in the vicinity of the Cs resonances. For comparison, the left hand side shows the measurements as in figure 6.8. The dielectric function of the atomic vapor $\epsilon_{1}(\omega)$ is calculated from equations 6.6, 6.7, and 6.8. In figure 6.10(a), the Cs resonance is at the blue foot of the first reflectivity peak. Referring to our explanation above, the change in $n_{v}^{\prime}$ over the Cs resonance will cause the measured narrowband reflectivity to move anticorrelated with $n_{v}^{\prime}$, while $n_{v}^{\prime \prime}$ causes them to be positive due to removal of destructive interference. These features are clearly observed: the shallow dip below resonance followed by a peak above resonance. When the Cs resonance is exactly in between the two broad reflectivity peaks (b), the Transfer-Matrix calculations show that while the change in $R(\omega)$ due to $n_{v}^{\prime}(\omega)$ is immaterial, $R(\omega)$ is predominantly influenced by $n_{v}^{\prime \prime}(\omega)$, and two large peaks are observed close to the Cs resonance. When the Cs resonance is at the red foot of the second reflectivity peak (c), the calculations reveal peaks in $R(\omega)$ below resonance, and troughs above resonance. $R(\omega)$ is now positively correlated with $n_{v}^{\prime}(\omega)$. For even further shifts, when the Cs resonance is at the red edge of the second reflectivity peak, two marked troughs are observed at resonance, attributed to the sensitivity of $R(\omega)$ on $n_{v}^{\prime \prime}(\omega)$.

The calculations agree with the measurements in three important respects: first, the lineshapes show the same features. For example, the trough below resonance (a) turns into a long tail (b). At higher temperatures, in (c), the initial trough has turned into a peak, which is followed by a trough. This trough becomes dominant, so much so that the initial peak disappears, and the spectrum only consists of troughs (d). Second, the baseline decreases from (a) to (b), and increases from (c) to (d). Third, the peaks have inverted in (d) relative to (b). From the good agreement we conclude that a strongly dispersive vapor inside a photonic structure has been probed. Our calculations differ in only one aspect from the measurement, this being the relative position of the exact resonance to the observed extrema. This discrepancy is attributed to pressure shift caused by remnant $N_{2}$ in the reservoir, which had not been evacuated prior to the experiment. From [201], the shift due to a $N_{2}$ buffer gas is $-10.97 \mathrm{MHz} / \mathrm{mbar}$, requiring a partial pressure of 27.3 mbar. This is in excellent agreement with the pressure deduced from later measuring the evacuated pressure during evacuation, yielding $27 \pm 10 \mathrm{mbar}$.

\subsection{Conclusions and recommendations}

Experiments of a hot vapor in a $\mathrm{SiO}_{2}$ photonic opal have been performed. We note that this is the first time ever evidence of sub-unity refractive in- 
dex in a photonic structure. The reflectivity peaks shifts in excess of $20 \%$ during the experiment, which is attributed to the reduction of the silica to $\mathrm{SiO}_{x}$. This shift changes the relative frequency of the Cs $D_{1}$ transition to the photonic features. During this time, the resonances change their strength, off-resonance reflectivity, and shape. A transfer-matrix calculation including the dispersion and absorption of two of the hyperfine transitions give excellent agreement to all observed features. We therefore conclude that the probed vapor was in the opal. Moreover, from the agreement we deduce that $n_{v}^{\prime}$ must have been less than unity for probe frequencies above resonance.

In the future, it is desirable to assert even more control over experimental conditions. The aggressive hot Cs vapor necessitated a number of precautions that indeed were taken. However, the leak due to corrosion of the copper gaskets in the reservoir was observed only late on, and will have to be prevented in the future. The leaks lead to a shift of the resonances. Measurement in a leak tight system might shed light upon theoretically predicted giant Lamb shifts [202]. Moreover, the film deposited on the reflecting optics must be avoided to properly assess the bandstructure of the sample. This could be achieved by means of a cold trap placed outside of the tank.

Although it was interesting to observe shifting reflectivity peaks, chemically inert (inverse) opals should be used. $\mathrm{Al}_{2} \mathrm{O}_{3}$ (alumina), which can be used as a backbone for inverse opals [203], is reported to be more inert than $\mathrm{SiO}_{2}$ [204]. To prevent optical absorption by the vapor of the probe beam, a method is needed to fabricate the crystals directly on the glass viewport.

While not essential for our experiments, the role of selective reflection of atoms in a photonic crystal should be studied, also to obtain information on how many atoms contribute to the optical density. Other exciting prospects include pulse delaying, see e.g. [205], performed on Cs vapor. A polarizable medium in a photonic crystal supports a polariton [32; 170], for which the wavenumber $k$ strongly depends on $n^{\prime}$. At the edges of the resonance, the bands in the crystal bend due to the presence of the atoms' dispersion. If the resonance is chosen e.g. below the first order stopgap, then the group velocity can greatly be decreased at these frequencies. Pulse delaying at the band edges of a photonic structure without a polarizable medium has been demonstrated elsewhere [173]. Finally, we propose to perform lifetime measurements on dense Cs in a photonic crystal with a high photonic strength, where strongly non-exponential decays have been predicted close to a band gap [206]. 

Appendix $A$

\section{Derivation of the position dependent effective dielectric constant of a colloid monolayer}

We describe how the optical properties of a three dimensional structure can be approximated by a one dimensional dielectric constant.

The colloids are placed on a silica substrate at $z=-r$. An incident plane wave in the $z$ direction sees the colloids' dielectric areal density $\epsilon(z)$ of the dielectric function $\epsilon(x, y, z)[207]$

$$
\epsilon(z)=1 / A \int_{A} d x d y \epsilon(x, y, z),
$$

where $\epsilon(z)$ can also be written in terms of the areal filling fraction

$$
\epsilon(z)=f(z) \epsilon_{2}+(1-f(z)) \epsilon_{1}(\omega)
$$

where the subscripts 2 and 1 indicate the silica and the atomic vapor, respectively. The dispersion of silica is not taken into account. For a colloid, $f(z)$ takes the form

$$
f(z)=\frac{\pi}{4}\left(r^{2}-z^{2}\right) .
$$

The factor $\pi r^{2} /(2 r)^{2}=\pi / 4$ results from the normalization condition that at $z=0$, the areal fraction of a circle to its smallest encompassing square is $\pi / 4$. $f(z)$ can be quantized in to $P$ slices, and the reflection and transmission coefficients can be calculated via the usual method of matrices [191; 208]. 



\section{Nederlandse samenvatting}

In dit proefschrift gaat het erom de optische eigenschappen van fotonische kristallen te beïnvloeden. In eerste instantie zal ik uitleggen wat fotonische kristallen zijn, verderop zal ik erop ingaan wat precies met 'optische eigenschappen' bedoeld is en hoe ze beïnvloed kunnen worden.

Iedereen weet wat kristallen zijn: vaste stoffen, die uit periodieke herhaling van atomen of moleculen bestaan. De afstand tussen de atomen is heel erg klein, ongeveer een nanometer. Een meter is een miljard, of duizend miljoen nanometer lang. Typische kristallen zijn ijs, kwarts of zout. Fotonische kristallen krijg je als de afstand tussen die atomen ongeveer duizend keer groter is. In dat geval is de afstand vergelijkbaar met de golflengte van zichtbaar licht, ongeveer 400 tot $800 \mathrm{~nm}$. Meestal worden fotonische kristallen dan ook niet van 'echte' atomen gemaakt (het is nogal lastig om echte atomen op hun plaats te houden), maar uit colloïdale bolletjes. Fotonische kristallen die uit bolletjes gemaakt zijn noemen wij opalen, omdat ze er opaalachtig uitzien: afhankelijk van de kijkrichting blijken ze een andere kleur te hebben. ${ }^{1}$ Zo een opaal is in figuur A.1 te zien. Een andere gebruikelijke manier om fotonische kristallen te maken is heel kleine stafjes loodrecht op elkaar te stapelen. Dit soort kristal noemen we fotonisch houtstapel kristal. Natuurlijk bestaan noch opalen noch de houtstapels uit hout.

${ }^{1} \mathrm{Om}$ precies te zijn, zeg je van perfekt periodieke structuren dat ze kleurenspel hebben (engl. iridescence), en van periodiek structuren met wanorde dat ze opaalachtig zijn. 


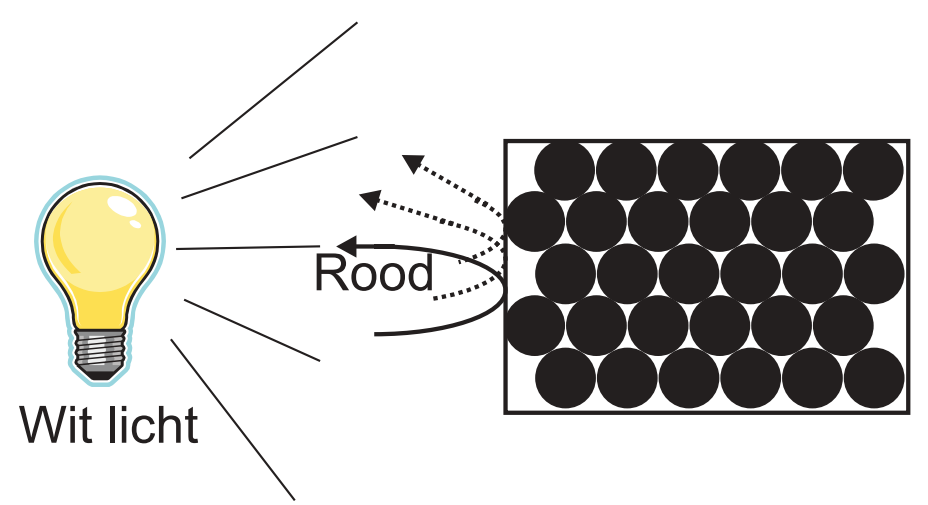

Figure A.1.: Schema van een opaal fotonisch kristal. Als zo'n fotonisch kristal met wit licht wordt verlicht, reflecteren bepaalde kleuren terug, in dit voorbeeld rood. Ondere andere kijkhoeken reflecteren andere kleuren.

Gebruikelijke materialen zijn halfgeleiders, materialen die licht heel sterk verstrooien. Het verstrooien (oftewel reflecteren) is ook noodzakelijk om het kristal ook echt fotonisch te maken. Hoe sterker de halfgeleider verstrooit, hoe sterker is het kristal fotonisch. De sterkte van de verstrooiing wordt door de brekingsindex bepaald. Hoe groter het verschil in de brekingsindex, hoe meer kleuren kunnen het kristal niet in, maar worden volledig teruggestrooid, zie figuur A.1. Dit frequentiegebied noemen we een stopband, en is een indicator voor de fotonische sterkte. Als de brekingsindex heel hoog is, ontstaat er een bandkloof, licht van bepaalde kleur kan dan in geen enkel richting propageren.

Fotonische kristallen zijn heel interessant en worden al ruim 20 jaar intensief onderzocht. Ze bieden een manier om licht op de nanoschaal te sturen. In dit proefschrift echter laten we op verschillende manieren de brekingsindex veranderen. Daardoor kun je de voortplanting van licht aktief beïnvloeden. Omdat fotonische kristallen heel gevoelig zijn voor veranderingen, lijkt het bijzonder interessant om dit te bestuderen. Een heel bijzondere fotonische kristal is een kristal met een gewenste defect, zie figuur A.2. Hierbij wordt de periodiciteit van het rooster op een plaats expres onderbroken. Wat verrassend is, is dat dit defekt de optische eigenschappen van het hele kristal verandert. Door het onderbroken rooster mag ineens wel licht in een stopband propageren, echter in een nauw frequentiegebied. Dit defekt werkt ook als trilholte: Het inkomende licht wordt een tijdje opgesloten. Als we nu de brekingsindex op korte termijn wijzigen, op tijdschalen die evenredig zijn met de opsloottijd, hebben we een heel interessant systeem. In hoofdstuk 3 laten we metingen zien hoe zich de trilholte in de tijd gedraagt. De 


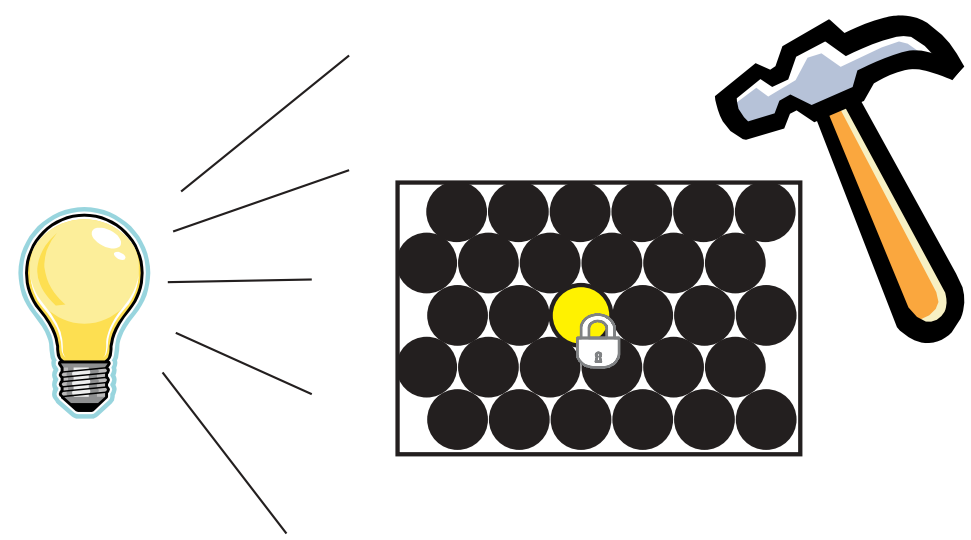

Figure A.2.: Trilholte in een fotonisch kristal. Licht wordt een tijdje in de trilholte opgesloten. Met behulp van een externe stimulus, zoals een korte pomp laser puls (gesymboliseerd als een hamer) kunnen we het licht bevrijden.

brekingsindex wordt met behulp van een sterke pomp laser puls binnen 100 fs veranderd (in die tijd propageert licht 30 miljoenste meter in de vrije ruimte). Met een tweede (probe) puls die net later komt kunnen we de frequentie van de trilholte meten. In figuur A.3, linker kolom, kunnen we zien wat er gebeurt: voor aankomst van de pomp gebeurt niets (a). Als de pomp aankomt, gebeurt er in eerste instantie niets (b). Maar een korte tijd later, worden de spiegels gedeeltelijk doorzichtig, en tegelijkertijd verandert de resonantie van de trilholte (c). Als je nog langer wacht herstelt het systeem zich weer in de uitgangssituatie (d-f). Met onze experimentele apparatuur kunnen we duidelijk en voor het allereerst zien hoe de frequentie van de trilholte verandert.

In hoofdstuk 4 bekijken we het opgesloten licht zelf. $\mathrm{Nu}$ we weten hoe zich de trilholte in de tijd gedraagt, willen we weten wat met het opgesloten licht gebeurt. Dit doen we door de volgorde van de twee pulsen om te draaien: de zwakke probe puls gaat eerst de trilholte in, pas een tijdje later wordt de trilholte dan door de pomp puls geschakeld, zie figuur A.3, rechter kolom. Als we met onze speciale meetapparatur hiernaar kijken, zien we duidelijk hoe de opgesloten puls vrij wordt gelaten. Met behulp van een fysische model bestaande uit een massa op een veer, kunnen we bijna alle verschijnsel begrijpen.

Hoofdstuk 5 gaat het over het schakelen van fotonische bandkloof kristallen. Deze zijn bijzonder interessant omdat ruim 20 jaar geleden voorspeld werd dat een lichtbron binnen zo een kristal volledig wordt onderdrukt, als in een kooi voor licht. Onze kristal is een silicium houtstapel kristal. Omdat sili- 


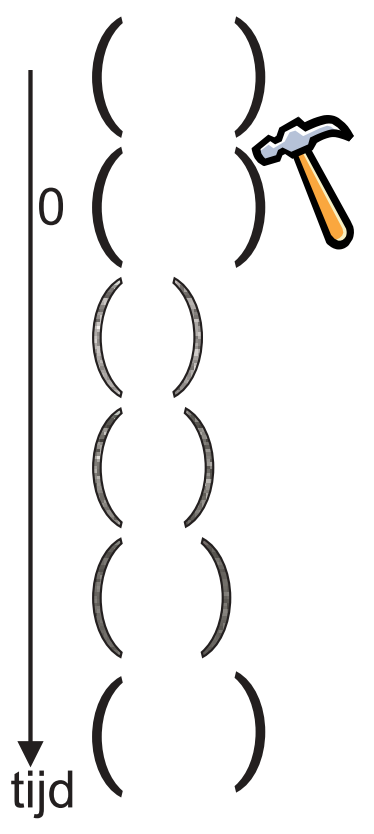

(a)

(b)

(c)

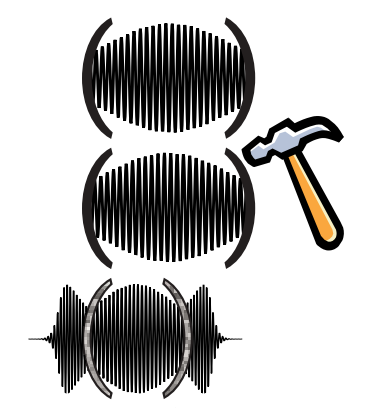

(d)

(e)

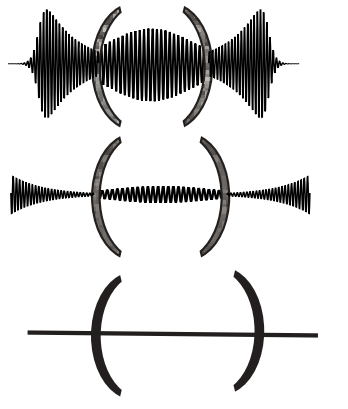

Figure A.3.: Schakeling van een trilholte in een fotonisch kristal. Linker kolom (hoofdstuk 3): Voor aankomst van de pomp (gesymboliseerd door de hamer, net als in figuur A.3) is de trilholte statisch (a). Bij aankomst van de pomp gebeurt er niet veel (b), maar wel een korte tijd later: niet alleen verplaatst de resonantie, maar ook de spiegels van de trilholte worden gedeeltelijk doorzichtig (c). De trilholte herstelt zich weer in de uitgangssituatie, en de spiegels worden minder doorzichtig (d-f). Rechte column (hoofdstuk 4): We bekijken een probe puls in een geschakelde trilholte. In (a) is er geen pomp puls aanwezig. In (b) is de probe een tijdje opgesloten geweest, en de pomp komt aan. Door de toegenomene doorzichtigheid van de spiegels, kan de probe pulse ontwijken (b). In (c-f) wordt de trilholte herstelt, maar de opgesloten puls is al lang ontsnapt. 


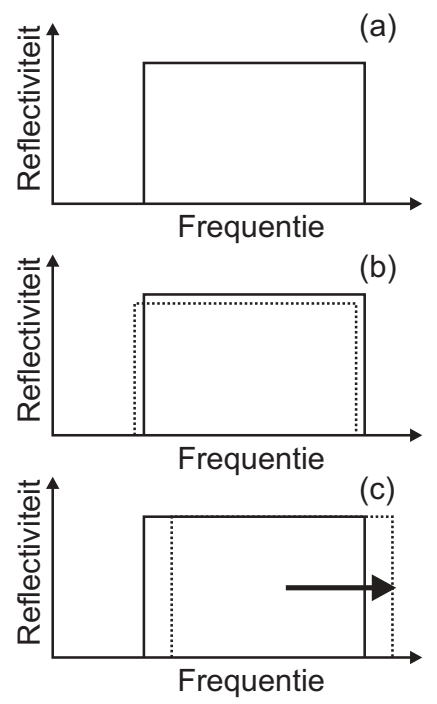

Figure A.4: Schakelen van een fotonisch houtstapel kristal. De hoge reflectiviteit in (a) is de brede stopband. Als de pomp tegelijkertijd komt met de probe, zien we stopband inzakken, maar ook naar het rode verschuiven (b). Als de probe later komt dan de pomp, zien we dat de hele stopband naar het blauw verplaatst.

cium een hoge brekingsindex heeft en er een voordelige verdeling van silicium is lucht is, heeft het houtstapel kristal een heel brede stopband. We hebben een schema van z'n brede stopband in figuur A.4(a) getekend. Net als in het vorig hoofdstuk doen we pomp-probe spectroscopie. Als de probe duidelijk later komt dan de pomp (c), zien we dat stopband verandert van frequentie naar het blauw. Het kristal verkleurt als het ware heel snel! Hoewel spectaculair, was dit effect al eerder waargenomen. Echter, als de pomp en probe tegelijk aankomen (b), nemen we waar dat enerzijds de probe enigzins geabsorbeerd wordt en anderzijds schakelt de stopband naar het rode, dus in de andere richting dan op langere probe vertragingen. Dit gezamenlijke gedrag geeft aanleiding tot volgende interpretatie: het schakelen naar rode komt door de tijdelijke, sterke elektrisch veld van het pomp, dat tijdens de aanwezigheid van de pomp het brekingsindex verandert. De rood schakeling is heel interessant omdat de tijdsduur daarvan alleen van de pomp afhangt. De schakelduratie kan dus in principe willekeurig snel gemaakt worden, gelegen voor ultiem snelle schakeltoepassingen. De aanwezigheid van absorptie komt door het feit dat de pomp de absorptie van de probe 'helpt'. Omdat en roodschakeling en absorptie sterk van zowel pomp als ook probe frequentie afhankelijk is, kunnen we door middel van een diagram aantonen voor welke pomp en probe frequenties een groot roodschakel effect mogelijk met minimale absorptie.

In het laatste hoofdstuk wijzigen we de brekingsindex van een fotonisch kristal op een heel ander manier. Het doel is om uit te vinden of we de fotonische sterkte kunnen verhogen door een ander medium met een resonant gedrag in het kristal te brengen. In figuur A.5 zien we een schema van een resonant medium in een fotonisch kristal en het effect hiervan. In de 


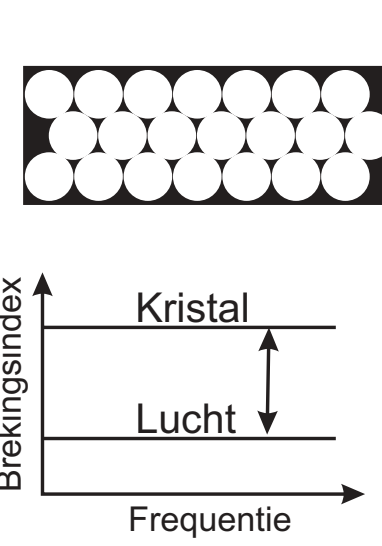

(a)
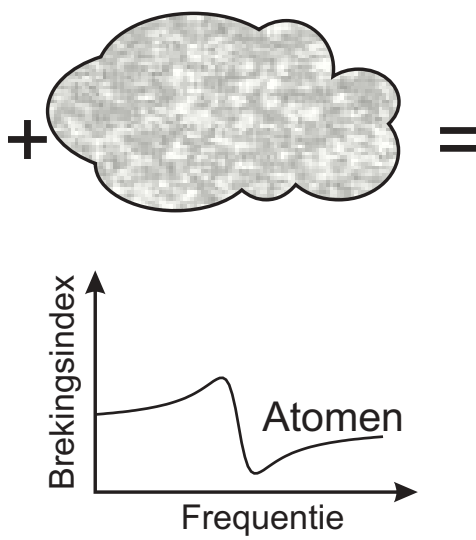

(b)
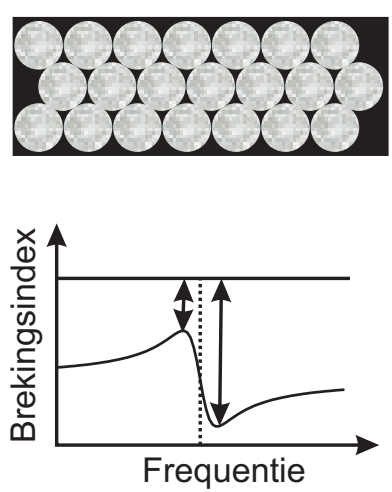

(c)

Figure A.5.: 'Schakelen' van een opaal. Bovenste rij: (a) Schema van een invers opaal. (b) Schema van een atomaire damp. (c) Atomaire damp in het kristal. Onderse rij: (a) Effective brekingsindex van een fotonisch kristal uitgezet tegen frequentie. (b) Brekingsindex van een atomaire damp. (c) Doordat de damp in het kristal is, wordt het verschil tussen de brekingsindices verandert. Onder resonantie (gestippeld lijn), is de fotonische sterkte lager, terwijl hij boven resonantie hoger is.

bovenste rij (a) zien we een invers opaal. Figuur A.5(b) laat een schema zien van een atomaire damp, die we als resonant medium gebruiken. Als de damp in het kristal is gediffundeerd, hebben we een situatie als in (c). In de onderste rij zijn de verwachte effecten getekend: De effectieve brekingsindex van het kristal is min of meer constant als functie van de frequentie en is dus onafhankelijk van de kleur, zie (a). Voor de damp geldt dit niet (b): het toont een dispersief gedrag. Als de damp in het kristal is, zien we dat de fotonische sterkte van het kristal met atomaire damp per frequentie heel erg verschillend is. Door het resonante gedrag van de atomen kunnen we de sterkte en verhogen en verlagen. Inderdaad is het ons als eersten gelukt om dit te bereiken. Als mogelijke vervolg experimenten stellen we voor om een kristal te gebruiken dat net geen bandkloof heeft. Door het infiltreren van een resonant medium maak je dan wel een bandkloof kristal bij een bepaalde frequentie.

Kortom, in dit proefschrift hebben we drie verschillende manieren bestudeerd om fotonische kristallen te schakelen. In eerste instantie is ons onderzoek erop gericht geweest om de verschijnselen te begrijpen en dit is grotendeels gelukt. Op grond van onze resultaten kan toch al met voorzichtige optimisme worden gespeculeerd over nieuwe toepassingen: hierbij wordt informatie gecodeerd als licht ultiem snel geschakeld en dus gemanipuleerd. 


\section{Dankwoord}

Danken doe je iemand om hem of haar te laten weten dat je blij bent met de onverdiende hulp die je hebt mogen ontvangen. Het dankwoord is terecht een deel van het proefschrift, omdat het de hulp op die plaats waardeert waar je het ontvangen hebt. In het Duits praat je van het 'zusammenschreiben' van een proefschrift (schrijven). Ik zal het hier ook over het 'zusammen schreiben' hebben (gezamenlijk schrijven).

In eerste instantie dank ik mijn promotor Willem. Willem, voor jou was nooit iets 'te erg', je kon goed tegen mijn soms pessimistische kant. We hebben na een wat droog begin heel goed samen gewerkt, vooral tijdens mijn verblijf op AMOLF. Ik heb heel erg veel van je geleerd: wat wetenschappelijk werk inhoudt, het goed schrijven van een verslag ('neem de lezer bij de hand!'), het systematisch werken, het presenteren van resultaten, het plannen, het uitkijken naar nieuwe resultaten ('Wat is het doel?'). Bedankt ook voor de ongedwongen, opgewekte sfeer die je in de groep verspreidt. Je letterlijke vertalingen van het Nederlands naar diverse Europese talen (en weer terug, met verrassende resultaten) zal ik nooit vergeten.

Ten tweede wil ik mijn co-promotor Allard bedanken. Bedankt voor de vele instructieve discussies, de wilde ideeën (die helaas niet altijd haalbaar waren), het zorgvuldig en gezamenlijk kijken naar data, en het grondig bespreken van experimentele en theoretische problemen. Ik dank je ook voor jouw inzet in het meedenken en oplossen van theoretische vragen. Jouw bek- 
waamheid om binnen twee minuten probleemloos over Italiaanse bruggenbouw in de 18de eeuw te praten, uit te rekenen hoe ver een auto op een Snickers rijdt, de geschiedenis achter de naamgeving van een onbekende formule te vertellen, en daarbij tenminste twee woorden (waarvan één een Russische wetenschapper) te gebruiken die ik niet kende, verbaasde me telkens weer.

Ad wil ik bedanken voor het zorgen voor een constructieve, discussie-rijke sfeer in de groep. Bovendien waardeerde ik het zeer dat je er nooit moe van werd onze presentaties kritisch te bekijken.

De groep waarin ik de afgelopen $41 / 2$ jaar gewerkt heb heeft een heel belangrijke rol gespeeld: In de groep konden plaatjes, data, mogelijkheden, maar ook kritische opmerkingen altijd bediscussieerd worden. Daar ben ik heel blij om. Toen ik begon waren er Peter Lodahl, Martijn, Karen, Arie, Ivo (succes met je nieuwe baan, waar die ook is, en hou je verscheidenheid bij!), Tom (wiens kookkunsten ik zeer gewaardeerd heb), Ivan (bedankt voor de leuke, Russisch-geïnspireerde avonden met Vita, Nina en jou), Boris (de supergoochelaar en gezellige kamergenoot), Lydia (die succesvol haar passie voor chemie aan ons door heeft gegeven, zie 'Chemie is leuk!'), Karin, Floris (wiens interesse voor de Italiaanse Renaissance me geïspireerd heeft ook ernaar te kijken), Willem Tjerkstra ('Heb je nog iets leuks met Linux gedaan?'), Léon 'MasterChief' Woldering (wiens genuanceerde, subtiele, ondersteunende en voorzichtige vragen naar het afmaken van mijn proefschrift me bemoedigden - not!), Bas (bedankt voor je computer ondersteuning), Raymond (je hebt mijn muzieksmaak duurzaam beïnvloed...), Karen Munnink (bedankt voor je administratieve hulp) en Tijmen: Ik wens je veel succes in Erlangen, bedankt voor je experimentele steun in de begintijd op AMOLF, en voor vele inspirerende discussies! Dan kamen er in de loop van de tijd Peter Zijlstra, Rob, Vitaly, prof. Valentin Freilikher, Wouter, Frerik, Elbert, Timo, Hannie, Bernard Kaas (bedankt voor je uitleg over anisotrope diffusie. Ik zal 51/20 nooit vergeten en ook niet dat Ivan de overloopkamer uitmoest omdat 'ie anders zou stikken van het lachen), Bart Hüsken (bedankt voor het lenen van de spectrometer en het uitlijnen ervan! Ook voor gesprekken tussendoor over de uitdagingen van een promotie). Cock, jouw praktische ondersteuning heb ik zeer gewaardeerd. Pepijn, je experimentele en theoretische expertise heeft het Cs experiment een heel stuk makkelijker gemaakt. Ik ben ervan overtuigt dat er nog iets leuks bij uitkomt. Femius Koenderink wil ik bedanken voor het beschikbaar stellen van zijn bandenstructuurprogramma 'HBand', waarmee ik twee figuren heb berekend. Klaus Boller wil ik bedanken voor het grondig bekijken van mijn proefschrift, en zijn commentaren. 
In Amsterdam op het AMOLF kwam ik een heel ander stel mensen en collega's tegen: Alex (bedankt voor de fijne samenwerking! Succes met de komende metingen! Het stelt me gerust dat de switchopstelling in vaardige handen is), Merel (onontbeerlijk bij NM producties), Oscar Bok, Adriaan Molenaar (ik ben onder de indruk dat je je passie voor het evangelie werkelijkheid hebt laten worden), Steven Kettelarij, Edwin en Iwert. In de Photon Scattering groep heb ik leren kennen Paolo, Sanli, Ramy, Otto, Pedro, Timmo, Patrick, Frans, Bergin, Rob Troost. Het was heel leerzaam om met jullie te discussiëren. Jord en Matteo, jullie waren twee fantastische kamergenoten. Daan wil ik bedanken voor interessante discussies over alles, en voor de spontane beslissing naar de zee te reizen! Bovendien wil ik Albert, Piet, Roudy, Richard en Rutger bedanken voor het hartelijke welkom op AMOLF, en voor de administratie die daar voor mij geregeld werd. Rob Kemper wil ik bedanken voor zijn hulp met laser gerelateerde dingen. Cher Jean-Michel et chère Yoanna, je voudrais vous remercier de la coopération productive - mais cette thèse de doctorat n'est point la dernière correspondence avec vous! Ook wil ik Huib Bakker bedanken voor de zeer inspirerende discussies die tot een deel van hoofdstuk 4 hebben geleid.

In Enschede ben ik redelijk snel in de christelijke studentenvereniging Agapé terecht gekomen. Ik wil de kringleden bedanken voor inspirerende kringavonden. Teun, bedankt voor de leerzame en inspirerende discussies over God. Ik waardeer het zeer dat je een van de paranimfen bent.

Als ich Steinfurt das erste Mal sah, war ich etwas verunsichert. Hier soll ich hinziehen? Aber es hat sich als eine sehr gute Entscheidung herausgestellt. Wir fühlen uns in der Borghorst Gemeinde wohl wohl (das münsterländische 'wohl'), und haben dort viele Freunde gefunden. Danke, dass Ihr es uns so einfach gemacht habt!

Tini und Ulf, auch Ihr kommt nicht ungeschoren davon. Die Tatsache, dass Ihr auch in der Gegend seid, hat vieles einfacher gemacht - an dieser Stelle danke ich Tini, dass ich doch das Flugzeug nach Cargèse bekommen habe! Danke für Eure Unterstützung in vielerlei Hinsicht. Tini - bald bist Du auch soweit! Viel Erfolg noch!

Wenn ich an Hamburg denke, fallen mir spontan einige Freunde ein, die mich und uns in dieser Zeit unterstützt haben: Achmed und Rebekka, Wiebke (na gut - eigentlich überall auf der Welt!) und Rahel. Danke Euch! Familie MacKenzie, auch Ihr habt zu dieser Dissertation beigetragen: Danke für die Anteilnahme! Mum, thank you for supporting me in this. You're special. Dad, thanks for proofreading (and certainly not only that!), and that you got me interested in science in the first place. Desweiteren will ich mich 
bei allen Lehrern bedanken, die meine Neugierde unterstützt haben.

Meine Süße, was hast Du mitgefiebert. Ich dank Dir so sehr, dass Du dran geblieben bist und dass Du mich so sehr unterstützt hast. Lass uns gemeinsam weitergehen! Ich lieb Dich:

\section{岸}

"In all these things we are more than conquerors through him who loved us."

Rm. 8:37 


\section{Bibliography}

[1] T. W. Ebbesen, H. J. Lezec, H. F. Ghaemi, T. Thio, and P. A. Wolff, Extraordinary optical transmission through sub-wavelength hole arrays, Nature 391, 667 (1998).

[2] V. G. Veselago, Electrodynamics of substances with simultaneously negative values of $\sigma$ and $\mu$, Sov. Phys. Uspeki-USSR 10, 509 (1968).

[3] J. B. Pendry, Negative refraction makes a perfect lens, Phys. Rev. Lett. 85, 3966 (2000).

[4] S. Iijima and T. Ishihashi, Single-shell carbon nanotubes of 1-nm diameter, Nature 363, 603605 (1993).

[5] F. J. P. Schuurmans, D. Vanmaekelbergh, J. van de Lagemaat, and A. Lagendijk, Strongly photonic macroporous GaP networks, Science 284, 141 (1999).

[6] W. L. Barnes, A. Dereux, and T. W. Ebbesen, Surface plasmon subwavelength optics, Nature 424, 824 (2003).

[7] K. J. Vahala, Optical microcavities, Nature 424, 839 (2003).

[8] J. D. Joannopoulos, S. G. Johnson, J. N. Winn, and R. D. Meade, Photonic Crystals - Molding the Flow of Light, 2nd edition ed. (Princeton University Press, Princeton, 2008).

[9] E. Yablonovitch, Photonic band-gap structures, J. Opt. Soc. Am. B 10, 283 (1993).

[10] W. L. Bragg, The Diffraction of Short Electromagnetic Waves by a 
Crystal, Proc. Camb. Phil. Soc. 17, 43 (1913).

[11] L. Rayleigh, On the Maintenance of Vibrations by Forces of Double Frequency, and on the Propagation of Waves through a medium endowed with a Periodic Structure, Phil. Mag. 24, 145 (1887).

[12] K. M. Ho, C. T. Chan, and C. M. Soukoulis, Existence of a photonic gap in periodic dielectric structures, Phys. Rev. Lett. 65, 3152 (1990).

[13] H. S. Sözüer, J. W. Haus, and R. Inguva, Photonic Bands - Convergence problems with the plane-wave method, Phys. Rev. B 45, 13962 (1992).

[14] S. Y. Lin, J. G. Fleming, D. L. Hetherington, B. K. Smith, R. Biswas, K. M. Ho, M. M. Sigalas, W. Zubrzycki, S. R. Kurtz, and J. Bur, A three-dimensional photonic crystal operating at infrared wavelengths, Nature 394, 251 (1998).

[15] S. Noda, K. Tomoda, N. Yamamoto, and A. Chutinan, Full ThreeDimensional Photonic Bandgap Crystals at Near-Infrared Wavelengths, Science 289, 604 (2000).

[16] E. Yablonovitch, Inhibited Spontaneous Emission in Solid-State Physics and Electronics, Phys. Rev. Lett. 58, 2059 (1987).

[17] P. Lodahl, A. F. van Driel, I. S. Nikolaev, A. Irman, K. Overgaag, D. Vanmaekelbergh, and W. L. Vos, Controlling the dynamics of spontaneous emission from quantum dots by photonic crystals, Nature 430, 654 (2004).

[18] M. Fujita, S. Takahashi, Y. Tanaka, T. Asano, and S. Noda, Simultaneous Inhibition and Redistribution of Spontaneous Light Emission in Photonic Crystals, Science 308, 1296 (2005).

[19] A. Kress, F. Hofbauer, N. Reinelt, M. Kaniber, H. J. Krenner, R. Meyer, G. Böhm, and J. J. Finley, Manipulation of the spontaneous emission dynamics of quantum dots in two-dimensional photonic crystals, Phys. Rev. B 71, 241304 (R) (2005).

[20] D. Englund, D. Fattal, E. Waks, G. Solomon, B. Zhang, T. Nakaoka, Y. Arakawa, Y. Yamamoto, and J. Vučković, Controlling the Spontaneous Emission Rate of Single Quantum Dots in a Two-Dimensional Photonic Crystal, Phys. Rev. Lett. 95, 013904 (2005).

[21] B. K. Tanner, Introduction to the Physics of Electrons in Solids (Cambridge University Press, Cambridge, 1995).

[22] A. Lagendijk and B. A. van Tiggelen, Resonant multiple scattering of light, Phys. Rep. 270, 143 (1996).

[23] S. John, Localization of light, Phys. Today 32 (1991).

[24] W. L. Vos, R. Sprik, A. van Blaaderen, A. Imhof, A. Lagendijk, 
and G. H. Wegdam, Strong effects of photonic band structures on the diffraction of colloidal crystals, Phys. Rev. B 53, 16231 (1996).

[25] R. J. Spry and D. J. Kosan, Theoretical Analysis of the Crystalline Colloidal Array Filter, Appl. Spect. 40, 782 (1986).

[26] A. F. Koenderink, Emission and Transport of Light in Photonic Crystals, Ph.D. thesis, University of Amsterdam, 2003.

[27] W. L. Vos, H. M. van Driel, M. Megens, A. F. Koenderink, and A. Imhof, in Proceedings of the NATO ASI "Photonic Crystals and Light Localization in the 21st century", Experimental probes of the optical properties of photonic crystals, edited by C. M. Soukoulis (Kluwer, Dordrecht, 2001), p. 181.

[28] M. Galli, F. Marabelli, and G. Guizzetti, Direct measurement of refractive-index dispersion of transparent media by white-light interferometry, Appl. Opt. 42, 3910 (2004).

[29] H. Míguez, V. Kitaev, and G. A. Ozin, Band spectroscopy of colloidal photonic crystal films, Appl. Phys. Lett. 84, 1239 (2004).

[30] C. G. Darwin, A Theory of X-ray Reflexion, Phil. Mag. 27, 675 (1914), as discussed in [9].

[31] W. L. Vos, M. Megens, C. M. van Kats, and P. Bösecke, Transmission and diffraction by photonic colloidal crystals, J. Phys.: Condens. Matter 8, 9503 (1996).

[32] D. V. van Coevorden, R. Sprik, A. Tip, and A. Lagendijk, Photonic band structure of atomic lattices, Phys. Rev. Lett. 77, 2412 (1996).

[33] Z. Zhang and S. Satpathy, Electromagnetic wave propagation in periodic structures: Bloch wave solution of Maxwell's equations, Phys. Rev. Lett. 65, 2650 (1990).

[34] K. M. Leung and Y. F. Liu, Full vector wave calculation of photonic band structures in face-centered-cubic dielectric media, Phys. Rev. Lett. 65, 2646 (1990).

[35] E. Yablonovitch, T. J. Gmitter, and K. M. Leung, Photonic band structure: The face-centered-cubic case employing nonspherical atoms, Phys. Rev. Lett. 67, 2295 (1991).

[36] K. M. Ho, C. T. Chan, C. M. Soukoulis, R. Biswas, and M. Sigalas, Photonic band gaps in three dimensions: New layer-by-layer periodic structures, Sol. St. Commun. 89, 413 (1994).

[37] E. Özbay, A. Abeyta, G. Tuttle, M. Tringides, R. Biswas, C. T. Chan, C. M. Soukoulis, and K. M. Ho, Measurement of a three-dimensional photonic band gap in a crystal structure made of dielectric rods, Phys. Rev. B 50, 1945 (1994). 
[38] J. G. Fleming and S. Lin, Three-dimensional photonic crystal with a stop band from 1.35 to $1.95 \mu \mathrm{m}$, Opt. Lett. 24, 49 (1999).

[39] L. A. Woldering, Fabrication of Photonic Crystals and Nanocavities, Ph.D. thesis, Universiteit Twente, 2008.

[40] P. Jiang, J. Bertone, K. Hwang, and V. Colvin, Single-Crystal Colloidal Multilayers of Controlled Thickness, Chem. Mater. 11, 2132 (1999).

[41] A. S. Dimitrov and K. Nagayama, Continuous convective assembling of fine particles into two-dimesional arrays on solid surfaces, Langmuir 12, 1303 (1996).

[42] D. J. Norris, E. G. Arlinghaus, L. Meng, R. Heiny, and L. E. Scriven, Opaline Photonic Crystals: How does self-assembly work?, Adv. Mat. 16, 1393 (2004).

[43] A. Turner Jr., E. B. Bradford, J. W. Vanderhoff, and G. Oster, Optical properties of uniform particle-size latexes, J. Opt. Soc. Am. 44, 603 (1954).

[44] R. Mayoral, J. Requena, J. S. Moya, C. López, A. Cintas, H. Miguez, F. Meseguer, L. Vázquez, M. Holgado, and A. Blanco, D Long-range ordering in a $\mathrm{SiO}_{2}$ submicrometer-sphere sintered superstructure, Adv. Mat. 9, 257 (1997).

[45] W. L. Vos, M. Megens, C. M. van Kats, and P. Bösecke, X-ray diffraction of photonic colloidal single crystals, Langmuir 13, 6004 (1997).

[46] J. E. G. J. Wijnhoven and W. L. Vos, Preparation of photonic crystals made of air spheres in titania, Science 281, 802 (1998).

[47] A. Blanco, E. Chomski, S. Grabtchak, M. Ibisate, S. John, S. W. Leonard, C. Lopez, F. Meseguer, H. Miguez, J. P. Mondia, G. A. Ozin, O. Toader, and H. M. van Driel, Large-scale synthesis of a silicon photonic crystal with a complete three-dimensional bandgap near 1.5micrometres, Nature 405, 437 (2000).

[48] Y. A. Vlasov, X. Z. Bo, J. C. Sturm, and D. J. Norris, On-chip natural assembly of silicon photonic bandgap crystals, Nature 414, 289 (2001).

[49] T. Rivera, J.-P. Debray, J. M. Gérard, B. Legrand, L. Manin-Ferlanzzo, and J. L. Oudar, Optical losses in plasma-etched AlGaAs microresonators using reflection spectroscopy, Appl. Phys. Lett. 74, 911 (1999).

[50] A. Löffler, J. P. Reithmaier, G. Sek, C. Hofmann, S. Reitzenstein, M. Kamp, and A. Forchel, Semiconductor quantum dot microcavity pillars with high-quality factors and enlarged dot dimensions, Appl. Phys. Lett. 86, 111105 (2005).

[51] S. Reitzenstein, C. Hofmann, A. Gorbunov, M. Strauß, S. H. Kwon, C. Schneider, A. Löffler, S. Höfling, M. Kamp, and A. Forchel, AlAs/GaAs 
micropillar cavities with quality factors exceeding 150.000, Appl. Phys. Lett. 90, 251109 (2007).

[52] A. F. Koenderink, L. Bechger, H. P. Schriemer, A. Lagendijk, and W. L. Vos, Broadband Fivefold Reduction of Vacuum Fluctuations Probed by Dyes in Photonic Crystals, Phys. Rev. Lett. 88, 143903 (2002).

[53] I. Nikolaev, P. Lodahl, and W. Vos, Quantitative analysis of directional spontaneous emission spectra from light sources in photonic crystals, Phys. Rev. A 71, 053813 (2005).

[54] M. S. Thijssen, R. Sprik, J. E. G. J. Wijnhoven, M. Megens, T. Narayanan, A. Lagendijk, and W. L. Vos, Inhibited Light Propagation and Broadband Reflection in Photonic Air-Sphere Crystals, Phys. Rev. Lett. 83, 2730 (1999).

[55] H. M. van Driel and W. L. Vos, Multiple Bragg wave coupling in photonic band-gap crystals, Phys. Rev. B 62, 9872 (2000).

[56] J.-F. Galisteo-López, E. Palacios-Lidón, E. Castillo-Martínez, and C. C. López, Optical study of the pseudogap in thickness and orientation controlled artificial opals, Phys. Rev. B 68, 115109 (2003).

[57] J.-G. Galisteo-López and C. López, High-energy optical response of artificial opals, Phys. Rev. B 70, 035108 (2004).

[58] A. F. Koenderink and W. L. Vos, Optical properties of real photonic crystals: anomalous diffuse transmission, J. Opt. Soc. Am. B 22, 1075 (2005).

[59] A. F. Koenderink, A. Lagendijk, and W. L. Vos, Optical extinction due to intrinsic structural variations of photonic crystals, Phys. Rev. B 72, 153102 (2005).

[60] J.-F. Galisteo-López and W. L. Vos, Angle resolved reflectivity of single-domain photonic crystals, effects of disorder, Phys. Rev. E 66, 036616 (2002).

[61] M. M. Sigalas, C. M. Soukoulis, C. T. Chan, and K. M. Ho, Electromagnetic-wave propagation through dispersive and absorptive photonic-band-gap materials, Phys. Rev. B 49, 11080 (1994).

[62] E. Yablonovitch, T. J. Gmitter, R. D. Meade, A. M. Rappe, K. D. Brommer, and J. D. Joannopoulos, Donor and Acceptor Modes in Photonic Band Structure, Phys. Rev. Lett. 67, 3380 (1991).

[63] I. Tarhan and G. H. Watson, Photonic Band Structure of fcc Colloidal Crystals, Phys. Rev. Lett. 76, 315 (1996).

[64] E. Flück, N. F. van Hulst, W. L. Vos, and L. Kuipers, Near-field optical investigation of three-dimensional photonic crystals, Phys. Rev. E 68, 
015601 (2003).

[65] K.-J. Boller, A. Imamoğlu, and S. E. Harris, Observation of Electromagnetically Induced Transparency, Phys. Rev. Lett. 66, 2593 (1991).

[66] T. Tanabe, M. Notomi, E. Kuramochi, A. Shinya, and H. Taniyama, Trapping and delaying photons for one nanosecond in an ultrasmall high-Q photonic-crystal nanocavity, Nat. Phot. 1, 49 (2007).

[67] G. F. Bohren and D. R. Huffman, Absorption and Scattering of Light by Small Particles (Wiley, New York, 1983).

[68] N. W. Ashcroft and N. D. Mermin, Solid State Physics, 1st ed. (Brooks Cole, New York, 1976).

[69] G. C. Cho, W. Kütt, and H. Kurz, Subpicosecond Time-Resolved Coherent-Phonon Oscillations in GaAs, Phys. Rev. Lett. 65, 764 (1990).

[70] W. A. Hügel, M. F. Heinrich, M. Wegener, Q. T. Vu, L. Bányai, and H. Haug, Photon Echoes from Semiconductor Band-to-Band Continuum Transitions in the Regime of Coulomb Quantum Kinetics, Phys. Rev. Lett. 83, 3313 (1999).

[71] R. Huber, F. Tauser, A. Brodschelm, M. Bichler, G. Abstreiter, and A. Leitenstorfer, How many-particle interactions develop after ultrafast excitation of an electronhole plasma, Nature 414, 286 (2001).

[72] L. Huang, J. P. Callan, E. N. Glezer, and E. Mazur, GaAs under Intense Ultrafast Excitation: Response of the Dielectric Function, Phys. Rev. Lett. 80, 185 (1998).

[73] K. Sokolowski-Tinten and D. von der Linde, Generation of dense electron-hole plasmas in silicon, Phys. Rev. B 61, 2643 (2000).

[74] D. Vujic and S. John, Pulse reshaping in photonic crystal waveguides and microcavities with Kerr nonlinearity: Critical issues for all-optical switching, Phys. Rev. A 72, 013807 (2005).

[75] M. Scalora, J. P. Dowling, C. M. Bowden, and M. J. Bloemer, Optical Limiting and Switching of Ultrashort Pulses in Nonlinear Photonic Band Gap Materials, Phys. Rev. Lett. 73, 1368 (1994).

[76] S. G. Hense and M. Wegener, Ultrafast switch-off of a vertical-cavity semiconductor laser, Phys. Rev. B 55, 9255 (1997).

[77] U. Keller, K. J. Weingarten, F. X. Kartner, D. Kopf, B. Braun, I. D. Jung, R. Fluck, C. Honninger, N. Matuschek, and J. A. der Au, Semiconductor saturable absorber mirrors (SESAM's) for femtosecond to nanosecond pulse generation in solid-state lasers, IEEE J. Sel. Top. Quant. Elec. 2, 435 (1996).

[78] H. M. Gibbs, T. N. C. Venkatesan, S. L. McCall, A. Passner, A. C. 
Gossard, and W. Wiegmann, Optical Modulation by optical tuning of a cavity, Appl. Phys. Lett. 34, 511 (1979).

[79] J. L. Jewell, S. L. McCall, A. Scherer, H. H. Houh, N. A. Whitaker, A. C. Gossard, and J. H. English, Transverse modes, waveguide dispersion, and 30 ps recovery in submicron GaAs/AlAs microresonators, Appl. Phys. Lett. 55, 22 (1989).

[80] T. Tanabe, M. Notomi, S. Mitsugi, A. Shinya, and E. Kuramochi, All-optical switches on a silicon chip realized using photonic crystal nanocavities, Appl. Phys. Lett. 87, 151112 (2005).

[81] V. R. Almeida, C. A. Barrios, R. R. Panepucci, and M. Lipson, Alloptical control of light on a silicon chip, Nature 431, 1081 (2004).

[82] Q. Xu, V. R. Almeida, and M. Lipson, Micrometer-scale all-optical wavelength converter on silicon, Opt. Lett. 30, 2733 (2005).

[83] P. M. Johnson, A. F. Koenderink, and W. L. Vos, Ultrafast switching of potonic density of states in photonic crystals, Phys. Rev. B. 66, 081102(R) (2002).

[84] M. Notomi and S. Mitsugi, Wavelength conversion via dynamic refractive index tuning of a cavity, Phys. Rev. A 73, 051803 (2006).

[85] P. Dong, S. F. Preble, J. T. Robinson, S. Manipatruni, and M. Lipson, Inducing Photonic Transitions between Discrete Modes in a Silicon Optical Microcavity, Phys. Rev. Lett. 100, 033904 (2008).

[86] B. P. J. Bret, T. L. Sonnemans, and T. W. Hijmans, Capturing a light pulse in a short high-finesse cavity, Phys. Rev. A 68, 023807 (2003).

[87] Q. Xu, P. Dong, and M. Lipson, Breaking the delay-bandwidth limit in a photonic structure, Nat. Phys. 31, 406 (2007).

[88] A. M. Yacomotti, F. Raineri, C. Cojocaru, P. Monnier, J. Levenson, and R. Raj, Nonadiabatic Dynamics of the Electromagnetic Field and Charge Carriers in High-Q Photonic Crystal Resonators, Phys. Rev. Lett. 96, 093901 (2006).

[89] T. S. Moss, Optical Properties of Semiconductors (Butterworth, Oxford, 1959), p. 48.

[90] H. A. Lorentz, Over de theorie der terugkaatsing en breking van het licht, Ph.D. thesis, Universiteit Leiden, 1875.

[91] J. M. Gérard, B. Sermage, B. Gayral, B. Legrand, E. Costard, and V. Thierry-Mieg, Enhanced Spontaneous Emission by Quantum Boxes in a Monolithic Optical Microcavity, Phys. Rev. Lett. 81, 1110 (1998).

[92] M. Soljačić and J. D. Joannopolous, Enhancement of nonlinear effects using photonic crystals, Nat. Mat. 3, 211 (2004).

[93] P. W. H. Pinkse, T. Fischer, P. Maunz, and G. Rempe, Trapping an 
atom with single photons, Nature 404, 365 (2000).

[94] J. Yu, S. Yuan, J.-Y. Gao, and L. Sun, Optical pulse propagation in a FabryPerot etalon: analytical discussion, J. Opt. Soc. Am. A 18, 2153 (2001).

[95] T. G. Euser, Ultrafast optical switching of photonic crystals, Ph.D. thesis, University of Twente, 2007.

[96] T. G. Euser, A. J. Molenaar, J. G. Fleming, B. Gralak, A. Polman, and W. L. Vos, All-optical ultrafast switching of Si woodpile photonic band gap crystals, Phys. Rev. B 77, 115214 (2008).

[97] J.-C. Diels and W. Rudolph, Ultrashort Laser Pulse Phenomena: Fundamentals, Techniques, and Applications on a Femtosecond Time Scale (Academic, San Diego, California, 1996).

[98] Measured with an OceanOptics USB2000 spectrometer.

[99] E. M. Purcell, , Phys. Rev. 69, 681 (1946).

[100] M. Först, J. Niehusmann, T. Plötzing, J. Bolten, T. Wahlbrink, C. Moormann, and H. Kurz, High-speed all-optical switching in ionimplanted silicon-on-insulator microring resonators, Opt. Lett. 32, 2046 (2007).

[101] F. Raineri, C. Cojocaru, R. Raj, P. Monnier, A. Levenson, C. Seassal, $\mathrm{X}$. Letartre, and P. Viktorovitch, Tuning a two-dimensional photonic crystal resonance via optical carrier injection, Opt. Lett. 30, 64 (2005).

[102] I. Fushman, E. Waks, D. Englund, N. Stoltz, P. Petroff, and J. Vučković, Ultrafast nonlinear optical tuning of photonic crystal cavities, Appl. Phys. Lett. 90, 091118 (2007).

[103] S. F. Preble, Q. Xu, and M. Lipson, Changing the colour of light in a silicon resonator, Nat. Phot. 1, 293 (2007).

[104] R. J. Warburton, C. S. Dürr, K. Karrai, J. P. Kotthaus, G. MedeirosRibeiro, and P. M. Petroff, Charged Excitons in Self-Assembled Semiconductor Quantum Dots, Phys. Rev. Lett. 79, 5282 (1997).

[105] J. M. Gérard, private communication, 2007.

[106] A. V. Uskov, E. P. O'Reilly, D. McPeake, N. N. Ledentsov, D. Bimberg, and G. Huyet, Carrier-induced refractive index in quantum dot structures due to transitions from discrete quantum dot levels to continuum states, Appl. Phys. Lett. 84, 272 (2004).

[107] R. P. Stanley, R. Houdré, U. Oesterle, M. Ilegems, and C. Weisbuch, Impurity modes in one-dimensional periodic systems: The transition from photonic band gaps to microcavities, Phys. Rev. A 48, 2246 (1993).

[108] M. D. Sturge, Optical absorption of Gallium Arsenide between 0.6 to 
2.75 eV, Phys. Rev. 127, 768 (1962).

[109] J. S. Blakemore, Semiconducting and other major properties of gallium arsenide, J. Appl. Phys. 53, R123 (1982).

[110] R. E. Fern and A. Onton, Refractive Index of AlAs, J. Appl. Phys. 42, 3499 (1971), note that the refractive index is given by $\operatorname{Re}\left(\epsilon^{1 / 2}\right)$.

[111] S. W. Leonard, H. M. van Driel, J. Schilling, and R. B. Wehrspohn, Ultrafast band-edge tuning of a two-dimensional silicon photonic crystal via free-carrier injection, Phys. Rev. B. 66, 161102(R) (2002).

[112] C. Becker, S. Linden, G. von Freymann, M. Wegener, N. Tétreault, E. Vekris, V. Kitaev, and G. A. Ozin, Two-color pump-probe experiments on silicon inverse opals, Appl. Phys. Lett. 87, 091111 (2005).

[113] T. Tanabe, K. Nishiguchi, A. Shinya, E. Kuramochi, H. Inokawa, and M. Notomi, Fast all-optical switching using ion-implanted silicon photonic crystal nanocavities, Appl. Phys. Lett. 90, 031115 (2007).

[114] M. E. Levinshtein and S. L. Rumyantsev, in Semiconductor Parameters, edited by M. E. Levinshtein, S. L. Rumyantsev, and M. Shur (World Scientific, Singapore, 1996).

[115] G. Fasol, W. Hackenberg, H. P. Hughes, K. Ploog, E. Bauser, and H. Kano, Continuous-wave spectroscopy of femtosecond carrier scattering in GaAs, Phys. Rev. B 41, 1461 (1990).

[116] H. M. van Driel, private communication, 2006.

[117] P. C. Becker, H. L. Fragnito, C. H. B. Cruz, R. L. Fork, J. E. Cunningham, J. E. Henry, and C. V. Shank, Femotosecond Photon Echoes from Band-to-Band Transitions in GaAs, Phys. Rev. Lett. 61, 1647 (1988).

[118] W. G. Spitzer and J. M. Whelan, Infrared Absorption and Electron Effective Mass in n-Type Gallium Arsenide, Phys. Rev. 114, 59 (1959).

[119] G. Segschneider, T. Dekorsy, H. Kurz, R. Hey, and K. Ploog, Energy resolved ultrafast relaxation dynamics close to the band edge of lowtemperature grown GaAs, Appl. Phys. Lett. 71, 2779 (1997).

[120] D. C. Hutchings and E. W. V. Stryland, Nondegenerate two-photon absorption in zinc blende semiconductors, J. Opt. Soc. Am. B 9, 2065 (1992).

[121] P. Dawson and K. Woodbridge, Effects of prelayers on minority-carrier lifetime in GaAs/AlGaAs double heterostructures grown by molecular beam epitaxy, Appl. Phys. Lett. 45, 1227 (1984), note that the first equation (unnumbered) should be: $\frac{1}{\tau_{m}}=\frac{1}{\tau_{R}}+\frac{1}{\tau_{N R}}+\frac{S_{1}+S_{2}}{d}$, stated correctly in [123].

[122] J. M. Gérard, B. Sermage, L. Bergomi, and J. Y. Marzin, Differentia- 
tion of the non radiative recombination properties of the two interfaces of MBE grown GaAs-GaAlAs quantum wells, Superlattices and Microstructures 8, 417 (1990).

[123] R. G. Nelson and R. J. Sobers, Interfacial recombination velocity in GaAlAs/GaAs heterostructures, Appl. Phys. Lett. 32, 761 (1978).

[124] B.-S. Song, S. Noda, T. Asano, and Y. Akahane, Ultra-high-Q photonic double-heterostructure nanocavity, Nat. Mat. 4, 207 (2005).

[125] P. Michler, A. Kiraz, C. Becher, W. V. Schoenfeld, P. M. Petroff, L. Zhang, E. Hu, and A. Imamoglu, A Quantum Dot Single-Photon Turnstile Device, Science 290, 2282 (2000).

[126] P. J. Harding, T. G. Euser, Y. R. Nowicki-Bringuier, J.-M. Gérard, and W. L. Vos, Ultrafast optical switching of planar GaAs/AlAs photonic microcavities, Appl. Phys. Lett. 91, 111103 (2007).

[127] H. J. Bakker, private communication, 2007.

[128] X. Hu, P. Jiang, C. Ding, H. Yang, and Q. Gong, Picosecond and low-power all-optical swtiching based on an organic photonic-bandgap microcavity, Nat. Phot. (2008).

[129] A. Chin, K. Y. Lee, B. C. Lin, and S. Horng, Picosecond photoresponse of carriers in Si ion-implanted Si, Appl. Phys. Lett. 69, 653655 (1996).

[130] K. Yoshino, S. Satoh, Y. Shimoda, Y. Kawagishi, K. Nakayama, and M. Ozaki, Tunable optical stop band and reflection peak in synthetic opal infiltrated with liquid crystal and conducting polymer as photonic crystal, Jpn. J. Appl. Phys. Part 2 38, L961 (1999).

[131] K. Busch and S. John, Liquid-Crystal Photonic-Band-Gap Materials: The Tunable Electomagnetic Vacuum, Phys. Rev. Lett. 83, 967 (1999).

[132] S. W. Leonard, J. P. Mondia, H. M. van Driel, O. Toader, S. John, K. Busch, A. Birner, U. Gosele, and V. Lehmann, Tunable twodimensional photonic crystals using liquid-crystal infiltration, Phys. Rev. B 61, 2389 (2000).

[133] A. D. Bristow, N. Rotenberg, and H. M. van Driel, Two-photon absorption and Kerr coefficients of silicon for 8502200 nm, Appl. Phys. Lett. 90, 191104 (2007).

[134] Q. Lin, J. Zhang, G. Piredda, R. W. Boyd, P. M. Fauchet, and G. P. Agrawal, Dispersion of silicon nonlinearities in the near infrared region, Appl. Phys. Lett. 91, 021111 (2007).

[135] M. Dinu, F. Quochi, and H. Garcia, Third-order nonlinearities in silicon at telecom wavelengths, Appl. Phys. Let. 82, 2954 (2003).

[136] S. R. Hastings, M. J. A. de Dood, H. Kim, W. Marshall, H. S. Eisenberg, and D. Bouwmeester, Ultrafast optical response of a high- 
reflectivity GaAs/AlAs Bragg mirror, Appl. Phys. Lett. 86, 031109 (2005).

[137] A. Haché and M. Bourgeois, Ultrafast all-optical switching in a siliconbased photonic crystal, Appl. Phys. Lett. 77, 4089 (2000).

[138] D. A. Mazurenko, R. Kerst, J. I. Dijkhuis, A. V. Akimov, V. G. Golubev, D. A. Kurdyukov, A. B. Pevtsov, and A. V. Sel'kin, Ultrafast Optical Switching in Three-Dimensional Photonic Crystals, Phys. Rev. Lett. 91, 213903 (2003).

[139] M. Sheik-Bahae, A. A. Said, T. H. Wei, D. J. Gahan, and E. W. van Stryland, Sensitive measurement of optical nonlinewarities using a single beam, IEEE J. Quantum Electron. 26, 760 (1991).

[140] A. Molenaar, Ultrafast optical switching of woodpile photonic crystals, Master's thesis, Complex Photonic Systems, Universiteit Twente, 2006.

[141] B. Gralak, M. J. A. de Dood, G. Tayeb, S. Enoch, and D. Maystre, Theoretical study of photonic band gaps in woodpile crystals, Phys. Rev. E 67, 066601 (2003).

[142] M. J. A. de Dood, B. Gralak, A. Polman, and J. G. Fleming, Superstructure and finite-size effects in a Si photonic woodpile crystal, Phys. Rev. B 67, 035322 (2003).

[143] T. G. Euser and W. L. Vos, Spatial homogeneity of optically switched semiconductor photonic crystals and of bulk semiconductors, J. Appl. Phys. 97, 043102 (2005).

[144] J. F. Reintjes and J. C. McGroddy, Indirect Two-Photon Transitions in Si at $1.06 \mu m$, Phys. Rev. Lett. 30, 901 (1973).

[145] H. K. Tsang, C. S. Wong, T. K. Liang, I. E. Day, S. W. Roberts, A. Harpin, J. Drake, and M. Asghari, Optical dispersion, two-photon absorption and self-phase modulation in silicon waveguides at $1.5 \mu \mathrm{m}$ wavelength, Appl. Phys. Lett. 80, 416 (2002).

[146] K. W.-K. Shung and Y. C. Tsai, Surface effects and bnad measurements in photonic crystals, Phys. Rev. B 48, 11265 (1993).

[147] Variable Angle Spectroscopic Ellipsometry Handbook (WVASE32), J. A. Woollam Co., Inc., 1987.

[148] C. Rotaru, S. Nastase, and N. Tomozeiu, Amorphous Phase Influence on the Optical Bandgap of Polysilicon, Phys. Stat. Sol. (a) 171, 365 (1999).

[149] H. Garcia and R. Kalyanaraman, Phonon-assisted two-photon absorption in the presence of a dc-field:the nonlinear Franz-Keldysch effect in indirect gap semiconductors, J. Phys. B 39, 2737 (2006). 
[150] D. C. Hutchins and E. W. V. Stryland, Nondegenerate two-photon abssorption in zinc blende semiconductors, J. Opt. Soc. Am. B. 9, 2065 (1992).

[151] M. Sheik-Bahae, D. J. Hagan, and E. W. V. Stryland, Dispersion and Band-Gap Scaling of the Electronic Kerr Effect in Solids Associated with Two-Photon Absorption, Phys. Rev. Lett. 65, 96 (1991).

[152] K. Ikeda, Y. Shen, and Y. Fainman, Enhanced optical nonlinearity in amorphous silicon and its application to waveguide devices, Opt. Expr. 15, 17761 (2007).

[153] E. Garmire, Nonlinear optics in semiconductors, Phys. Today 42 (1994).

[154] C. Klingshirn, Semiconductor Optics (Springer, Berlin, 2005).

[155] M. Sheik-Bahae, J. Wang, and E. W. V. Stryland, Nondegenerate optical Kerr effect in semiconductors, IEEE J. Quant. Elect. 30, 249 (1994).

[156] E. Fermi, Quantum Theory of Radiation, Rev. Mod. Phys. 4, 87 (1932).

[157] D. Kleppner, Inhibited Spontaneous Emission, Phys. Rev. Lett. 47, 233 (1981).

[158] K. H. Drexhage, H. Kuhn, and F. P. Schafer, Variation of fluorescence decay time of a molecule in front of a mirror, Ber. d. Bunsen-Ges. f. Phys. Chem. 72, 329 (1968).

[159] R. M. Amos and W. L. Barnes, Modification of spontaneous emission lifetimes in the presence of corrugated metallic surfaces, Phys. Rev. B 59, 7708 (1999).

[160] G. Lecamp, P. Lalanne, and J. P. Hugonin, Very large spontaneousemission beta factors in photonic-crystal waveguides, Phys. Rev. Lett 99, 023902 (2007).

[161] S. J. Myers, D. P. Fussell, J. M. Dawes, E. Mägi, R. C. McPhedran, B. J. Eggleton, and C. M. de Sterke, Manipulation of spontaneous emission in a tapered photonic crystal fiber, Opt. Express 14, 12439 (2006).

[162] P. Goy, J. M. Raimond, M. Gross, and S. Haroche, Observation of cavity-enhanced single-atom spontaneous emission, Phys. Rev. Lett. 50, 1903 (1983).

[163] M. Trupke, J. Goldwin, B. Darquié, G. Dutier, S. Eriksson, J. Ashmore, and E. A. Hinds, Atom Detection and Photon Production in a Scalable, Open, Optical Microcavity, Phys. Rev. Lett. 99, 063601 (2007).

[164] T. Yoshie, A. Scherer, J. Hendrickson, G. Khitrova, H. M. Gibbs, 
G. Rupper, C. Ell, O. B. Shchekin, and D. G. Deppe, Vacuum Rabi splitting with a single quantum dot in a photonic crystal nanocavity, Nature 432, 200 (2004).

[165] J. P. Reithmaier, S. Sek, A. Loffler, C. Hofmann, S. Kuhn, S. Reitzenstein, L. V. Keldysh, V. D. Kulakovskii, T. L. Reinecke, and A. Forchel, Strong coupling in a single quantum dot-semiconductor microcavity system, Nature 432, 197 (2004).

[166] E. Peter, P. Senellart, D. Martrou, A. Lemaitre, J. Hours, J. M. Gérard, and J. Bloch, Exciton-photon strong-coupling regime for a single quantum dot embedded in a microcavity, Phys. Rev. Lett. 95, 067401 (2005).

[167] T. Aoki, B. Dayan, E. Wilcut, W. P. Bowen, A. S. Parkins, T. J. Kippenberg, K. J. Vahala, and H. J. Kimble, Observation of strong coupling between one atom and a monolithic microresonator, Nature 443, 671 (2006).

[168] D. Englund, A. Faraon, I. Fushman, N. Stoltz, P. Petroff, and J. Vuckovic, Controlling cavity reflectivity with a single quantum dot, Nature 450, 857 (2007).

[169] N. Eradat, A. Y. Sivachenko, M. E. Raikh, Z. V. Vardeny, A. A. Zakhidov, and R. H. Baughman, Evidence for braggoriton excitations in opal photonic crystals infiltrated with highly polarizable dyes, Appl. Phys. Lett. 80, 3491 (2002).

[170] A. Y. Sivachenko, M. E. Raikh, and Z. V. Vardeny, Excitations in photonic crystals infiltrated with polarizable dye, Phys. Rev. A 64, 013809 (2001).

[171] V. Kuzmiak, A. A. Maradudin, and A. R. McGurn, Photonic band structures of two-dimensional systems fabricated from rods of a cubic polar crystal, Phys. Rev. B 55, 4298 (1997).

[172] D. Kang, J. E. Maclennan, N. A. Clark, A. A. Zakjidov, and R. H. Baughman, Electro-optic Behavior of Liquid-Crystal-Filled Silica Opal Photonic Crystals: Effect of Liquid-Crystal Alignment, Phys. Rev. Lett. 86, 4052 (2001).

[173] A. Imhof, W. L. Vos, R. Sprik, and A. Lagendijk, Large Dispersive Effects near the Band Edges of Photonic Crystals, Phys. Rev. Lett. 83, 2942 (1999).

[174] M. Megens, J. E. G. J. Wijnhoven, A. Lagendijk, and W. L. Vos, Fluorescence lifetimes and linewidths of dye in photonic crystals, Phys. Rev. A 59, 4727 (1999).

[175] D. J. Norris and M. G. Bawendi, Measurement and assignment of the 
size-dependent optical spectrum in CdSe quantum dots, Phys. Rev. B 53, 16338 (1996).

[176] M. Weidemüller, A. Hemmerich, A. Gorlitz, T. Esslinger, and T. W. Hänsch, Bragg-diffraction in an atomic lattice bound by light, Phys. Rev. Lett. 75, 4583 (1995).

[177] G. Birkl, M. Gatzke, I. H. Deutsch, S. L. Rolston, and W. D. Phillips, Bragg Scattering from Atoms in Optical Lattices, Phys. Rev. Lett. 75, 2823 (1995).

[178] M. Greiner, O. Mandel, T. Esslinger, T. W. Hänsch, and I. Bloch, Quantum phase transition from a superfluid to a Mott insulator in a gas of ultracold atoms, Nature 415, 39 (2002).

[179] R. J. Rafac, C. E. Tanner, A. E. Livingston, and H. G. Berry, Fast-beam laser lifetime measruements of the cesium $6 p^{2} P_{1 / 2,3 / 2}$ states, Phys. Rev. A 60, 3648 (1999), as discussed by [187].

[180] L. Young, W. T. Hill III, S. J. Sibener, S. D. Price, C. E. Tanner, C. E. Wieman, and S. R. Leone, Precision lifetime measurements of Cs $6 p^{2} P_{1 / 2}$ and $6 p^{2} P_{3 / 2}$ levels by single-photon counting, Phys. Rev. A 50, 2174 (1994), as discussed by [187].

[181] R. J. Rafac and C. E. Tanner, Measurement of the ratio of the cesium D-line transition strengths, Phys. Rev. A 58, 1087 (1998), as discussed by $[187]$.

[182] in General Conference on Weights and Measures (CGPM) (CGPM, Paris, 1967).

[183] U. Fano and L. Fano, Physics of Atoms and Molecules (The University of Chicago Press, Chicago, 1972).

[184] R. Grimm, M. Weidemüller, and Y. B. Ovchinnikov, Optical Dipole Traps for Neutral Atoms, Advances in Atomic, Molecular and Optical Physics 42, 95 (2000).

[185] W. Demtröder, Laser Spektroskopie (Springer, Berlin, 1996).

[186] C. Wieman and T. W. Hänsch, Doppler-free laser polarization spectroscopy, Phys. Rev. Lett. 36, 1170 (1976).

[187] D. A. Steck, Technical report, Los Alamos National Laboratory, Los Alamos, NM 87545 (unpublished).

[188] C. Kittel and H. Kroemer, Thermal Physics, 2nd ed. (Freeman, Virginia, 1980), pp. 281-282.

[189] Handbook of Chemistry and Physics, 76th edition ed., edited by D. R. Lide (CRC, Boca Raton, Florida, 1995-1996).

[190] C. Chen and A. Phelps, Self-broadening of Cesium Resonance Lines at 8521 and $8944 \dot{A}$, Phys. Rev. 173, 62 (1968). 
[191] M. Born and E. Wolf, in Principles of Optics, edited by 6th edition (Cambridge University Press, Cambridge, 1997).

[192] J. Guo, J. Cooper, A. Gallagher, and M. Lewenstein, Theory of selective reflection spectroscopy, Opt. Commun. 110, 197 (1994).

[193] P. Wang, A. Gallagher, and J. Cooper, Selective Reflection by Rb, Phys. Rev. A 56, 1598 (1997).

[194] J. P. Woerdman and M. F. H. Schuurmans, Spectral narrowing of selective reflection from sodium, Opt. Comm. 14, 248 (1975).

[195] S. M. Weiss and P. M. Fauchet, Porous Silicon One-Dimensional Photonic Crystals for Optical Signal Modulation, IEEE J. Sel. Top. Quant. Elec. 12, 1514 (2006).

[196] G. Lewin, Fundamentals of Vacuum Science and Technology (McGrawHill, Columbus, 1965).

[197] R. Frueholz and M. Wun-Fogle, , J. Am. Ceram. Soc. 66, 605 (1983).

[198] E. Jahier, J. Guéna, P. Jacquier, M. Lintz, and M. A. Bouchiat, Implementation of a sapphire cell with external electrodes for laser excitation of a forbidden atomic transition in a pulsed E-field, Europ. Phys. J. D 13, 221 (2001).

[199] P. Pascal, Nouveau traité de chimie minérale, Tome III (Masson, Paris, 1958).

[200] S. H. Wemple, Refractive-index behavior of amorphous semiconductors and glasses, Phys. Rev. B 7, 3767 (1973).

[201] A. Andalkar and R. Warrington, High-resolution measurement of the pressure broadening and shift of the $\mathrm{Cs} \mathrm{D}_{1}$ and $\mathrm{D}_{2}$ lines by $\mathrm{N}_{2}$ and $\mathrm{He}$ buffer gases, Phys. Rev. B 65, 032708 (2002).

[202] X. H. Wang, Y. S. Kivshar, and B. Y. Gu, Giant Lamb shift in photonic crystals, Phys. Rev. Lett. 93, 073901 (2004).

[203] L. Bechger, Synthesis and Flyorescence of Opal 83 Air-Sphere Photonic Crystals, Ph.D. thesis, Universiteit Twente, 2003.

[204] J. Guéna, D. Chauvat, P. Jacquier, E. Jahier, M. Lintz, S. Sanguinetti, A. Wasan, M. A. Bouchiat, A. V. Papoyan, and D. Sarkisyan, New Manifestation of Atomic Parity Violation in Cesium: A Chiral Optical Gain Induced by Linearly Polarized $6 S-7 S$ Excitation, Phys. Rev. Lett. 90, 143001 (2003).

[205] R. M. Camacho, M. V. Pack, J. C. Howell, A. Schweinsberg, and R. W. Boyd, Wide-Bandwidth, Tunable, Multiple-Pulse-Width Optical Delays Using Slow Light in Cesium Vapor, Phys. Rev. Lett. 98, 153601 (2007).

[206] S. John and T. Quang, Spontaneous emission near the edge of a photonic band gap, Phys. Rev. A 50, 1764 (1994). 
[207] A. P. Mosk, private communication (unpublished).

[208] A. Yariv and P. Yeh, Optical Waves in Crystals (Wiley, New York, 1984). 
